NATIONAL LABORATORY

MANAGED BY UT-BATTELLE

FOR THE DEPARTMENT OF ENERGY

\title{
GUIDE TO PERFORMING COMPUTATIONAL ANALYSIS OF CRITICALITY ACCIDENT ALARM SYSTEMS
}

August 30, 2013

Prepared by

Thomas M. Miller

Douglas. E. Peplow

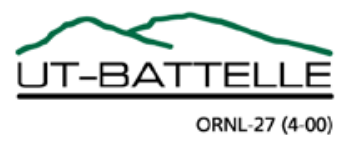




\title{
DOCUMENT AVAILABILITY
}

Reports produced after January 1, 1996, are generally available free via the U.S. Department of Energy (DOE) Information Bridge.

Web site http://www.osti.gov/bridge

Reports produced before January 1, 1996, may be purchased by members of the public from the following source.

\author{
National Technical Information Service \\ 5285 Port Royal Road \\ Springfield, VA 22161 \\ Telephone 703-605-6000 (1-800-553-6847) \\ TDD 703-487-4639 \\ Fax 703-605-6900 \\ E-mail info@ntis.gov \\ Web site http://www.ntis.gov/support/ordernowabout.htm
}

Reports are available to DOE employees, DOE contractors, Energy Technology Data Exchange (ETDE) representatives, and International Nuclear Information System (INIS) representatives from the following source.

Office of Scientific and Technical Information

P.O. Box 62

Oak Ridge, TN 37831

Telephone 865-576-8401

Fax 865-576-5728

E-mail reports@osti.gov

Web site http://www.osti.gov/contact.html

This report was prepared as an account of work sponsored by an agency of the United States Government. Neither the United States Government nor any agency thereof, nor any of their employees, makes any warranty, express or implied, or assumes any legal liability or responsibility for the accuracy, completeness, or usefulness of any information, apparatus, product, or process disclosed, or represents that its use would not infringe privately owned rights. Reference herein to any specific commercial product, process, or service by trade name, trademark, manufacturer, or otherwise, does not necessarily constitute or imply its endorsement, recommendation, or favoring by the United States Government or any agency thereof. The views and opinions of authors expressed herein do not necessarily state or reflect those of the United States Government or any agency thereof. 
Reactor and Nuclear Systems Division

\section{GUIDE TO PERFORMING COMPUTATIONAL ANALYSIS OF CRITICALITY ACCIDENT ALARM SYSTEMS}

Thomas M. Miller

Douglas E. Peplow

Date published: August 30, 2013

Prepared by

OAK RIDGE NATIONAL LABORATORY

Oak Ridge, Tennessee 37831-6285

managed by

UT-BATTELLE, LLC

for the

U.S. DEPARTMENT OF ENERGY

under contract DE-AC05-00OR22725 



\section{TABLE OF CONTENTS}

LIST OF FIGURES . $\mathrm{V}$

LIST OF TABLES vii

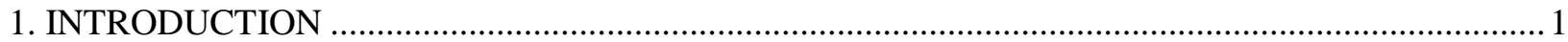

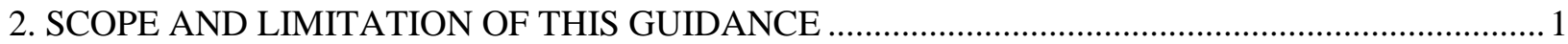

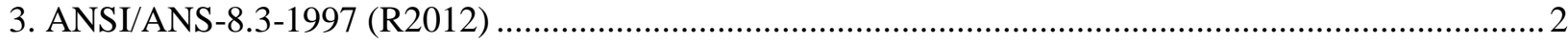

4. BASICS OF TRANSPORT CALCULATIONS REQUIRED TO PERFORM CAAS ANALYSIS ........ 3

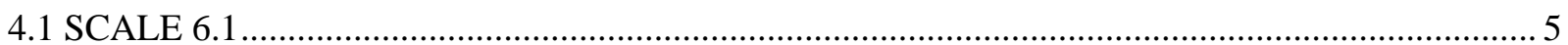

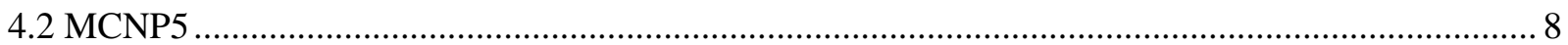

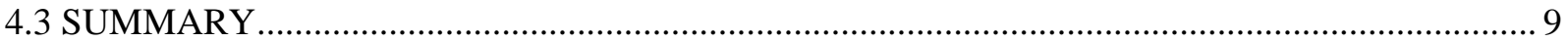

5. DETERMINING THE MINIMUM ACCIDENT OF CONCERN …............................................... 9

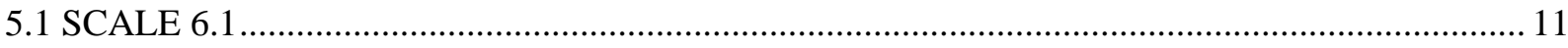

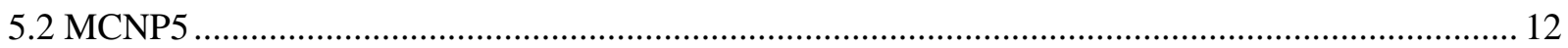

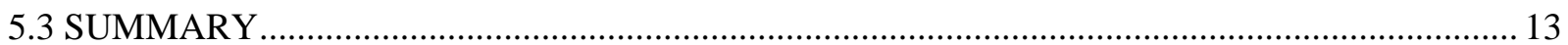

6. POTENTIAL SIMPLIFICATION OF THE MINIMUM ACCIDENT OF CONCERN........................ 14

7. CALCULATING THE RESPONSE OF A CAAS DETECTOR …................................................. 15

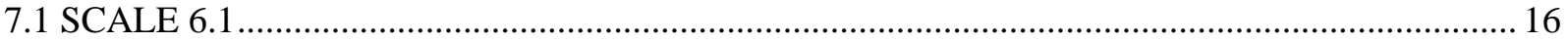

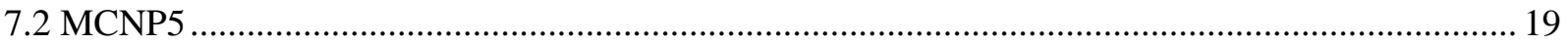

7.3 SCALE 6.1 - CRITICALITY TREATED AS A POINT .......................................................... 19

7.4 MCNP5 - CRITICALITY TREATED AS A POINT …............................................................... 22

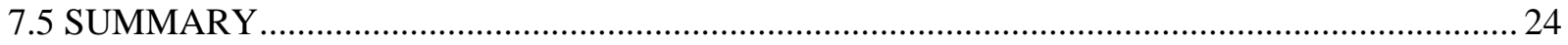

8. EVALUATING THE COVERAGE OF A CAAS DETECTOR .....................................................26

8.1 SCALE 6.1 - CAAS COVERAGE EVALUATED BY A FORWARD MESH TALLY ................ 27

8.2 MCNP5 - CAAS COVERAGE EVALUATED BY A FORWARD MESH TALLY ..................... 28

8.3 SCALE 6.1 - CAAS COVERAGE EVALUATED BY AN ADJOINT MESH TALLY ................ 29

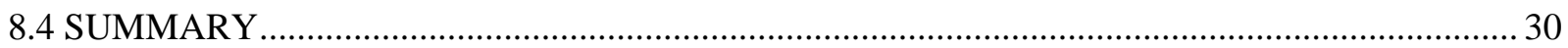

9. CAAS PLACEMENT ANALYSIS STRATEGY AND EXAMPLES ............................................... 33

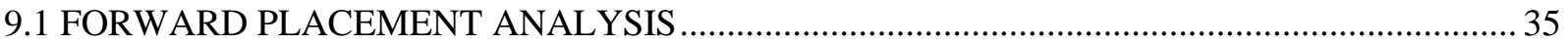

9.1.1 Approach 1: Forward Simulation, Analog MC, Standard Tallies ............................................. 35

9.1.2 Approach 2: Forward Simulation, Analog MC, Mesh Tally.................................................. 36

9.1.3 Approach 3: Forward Simulation, CADIS, Standard Tallies............................................... 37

9.1.4 Approach 4: Forward Simulation, FW-CADIS, Mesh Tally ................................................. 37

9.1.5 Detector Placement using Forward Approaches ....................................................................... 40

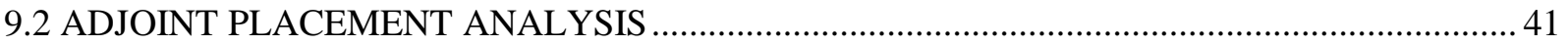

9.2.1 Approach 5: Adjoint Simulation, Analog MC, Standard Tallies .............................................. 41

9.2.2 Approach 6: Adjoint Simulation, Analog MC, Mesh Tally ................................................... 43 
9.2.3 Approach 7: Adjoint Simulation, CADIS, Standard Tallies ................................................. 43

9.2.4 Approach 8: Adjoint Simulation, FW-CADIS, Mesh Tally …................................................ 43

9.2.5 Adjoint Approaches with Denovo-Only Calculations ............................................................. 43

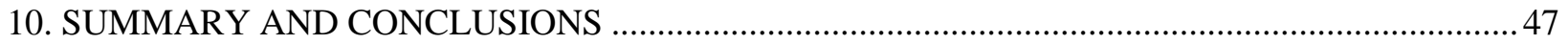

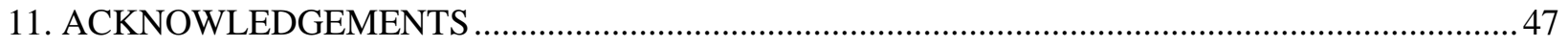

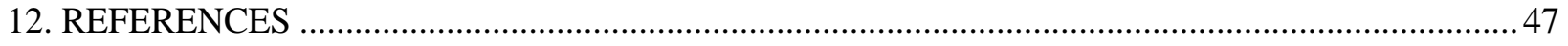

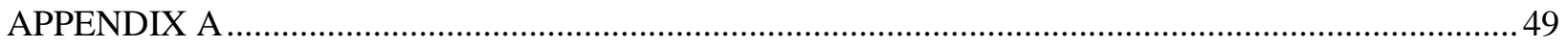

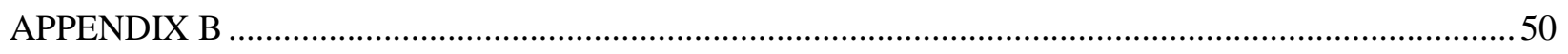

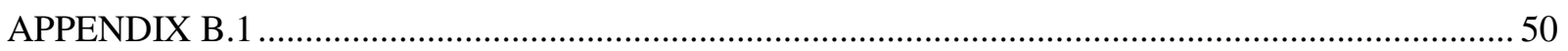

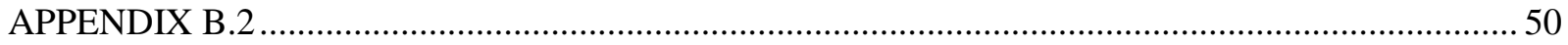

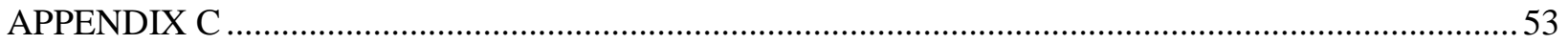




\section{LIST OF FIGURES}

Figure 1. Computational flow of a CAAS detector response evaluation using a fixed-source..................... 4

Figure 2. Computational flow of a CAAS detector response evaluation without a fixed-source................. 4

Figure 3. Block building with Jezebel, top half removed. .................................................................. 11

Figure 4. Block building with Jezebel and two-meter tally sphere, front half removed........................... 11

Figure 5. Block building with Jezebel and CAAS detector, roof and front wall removed. ....................... 15

Figure 6. Grid geometry employed by Denovo deterministic calculations............................................. 18

Figure 7. Comparison of SCALE and MCNP neutron leakage spectra................................................. 21

Figure 8. Comparison of SCALE and MCNP photon leakage spectra.................................................... 22

Figure 9. Plan view of forward calculated neutron air kerma rate map $(\mathrm{z}=100, \mathrm{~Gy} / \mathrm{min})$....................... 31

Figure 10. Elevation view of forward calculated neutron air kerma rate map ( $y=300, \mathrm{~Gy} / \mathrm{min})$.............. 31

Figure 11. Plan view of forward calculated photon air kerma rate map ( $\mathrm{z}=100, \mathrm{~Gy} / \mathrm{min})$...................... 31

Figure 12. Elevation view of forward calculated photon air kerma rate map ( $y=300, G y / m i n) \ldots \ldots \ldots \ldots . . . . .31$

Figure 13. Plan view of adjoint calculated neutron air kerma rate map ( $\mathrm{z}=100, \mathrm{~Gy} / \mathrm{min}) \ldots \ldots \ldots \ldots \ldots \ldots \ldots \ldots . . . . . . . .32$

Figure 14. Elevation view of adjoint calculated neutron air kerma rate map ( $y=300, G y / m i n)$............... 32

Figure 15. Plan view of adjoint calculated photon air kerma rate map ( $\mathrm{z}=100$, Gy/min)....................... 32

Figure 16. Elevation view of adjoint calculated photon air kerma rate map ( $y=300, G y / m i n)$................ 32

Figure 17. The storage room (ceiling and walls removed for visualization) showing the detector locations (blue, $3 \mathrm{~m}$ above floor) and some accident locations (red). Accident locations B and C are between the

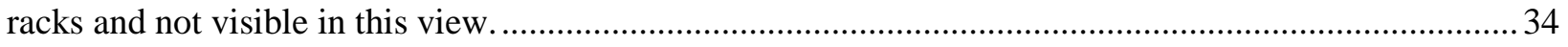

Figure 18. Overhead view of the storage room showing three detector locations (blue) and four accident

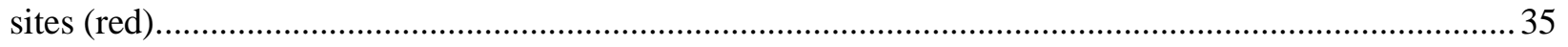

Figure 19. Close-up view of the double-sided storage bin, with sides removed....................................... 35

Figure 20. Gamma dose mesh tally from source A (forward, analog) ................................................... 36

Figure 21. Gamma dose mesh tally from source B (forward, analog) ................................................. 36

Figure 22. Gamma dose mesh tally from source C (forward, analog) ............................................... 37

Figure 23. Gamma dose mesh tally from source D (forward, analog) ................................................... 37

Figure 24. Gamma dose mesh tally from source A (forward, FW-CADIS) ........................................... 38

Figure 25. Gamma dose mesh tally from source B (forward, FW-CADIS). ........................................... 38

Figure 26. Gamma dose mesh tally from source C (forward, FW-CADIS) ............................................ 38 
Figure 27. Gamma dose mesh tally from source D (forward, FW-CADIS) .......................................... 38

Figure 28. Gamma dose mesh tally from source A (forward, modified FW-CADIS)............................. 39

Figure 29. Gamma dose mesh tally from source B (forward, modified FW-CADIS)............................. 39

Figure 30. Gamma dose mesh tally from source C (forward, modified FW-CADIS)............................ 40

Figure 31. Gamma dose mesh tally from source D (forward, modified FW-CADIS)............................. 40

Figure 32. Areas that would alarm for accident site A. ........................................................................ 40

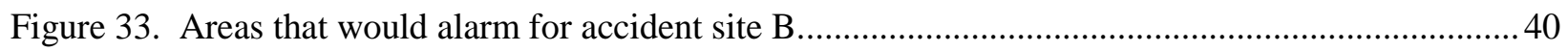

Figure 34. Areas that would alarm for accident site C......................................................................... 41

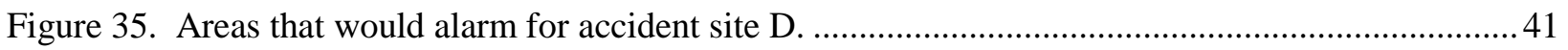

Figure 36. The number of accidents that can be seen from any given position at $\mathrm{z}=290 \mathrm{~cm}$................ 41

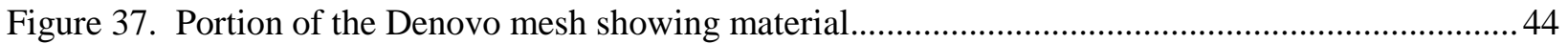

Figure 38. Total adjoint scalar flux as computed by Denovo with source in detector $1(\mathrm{z}=100 \mathrm{~cm}) \ldots \ldots . . .45$

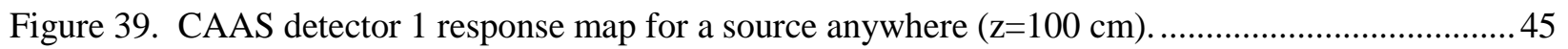

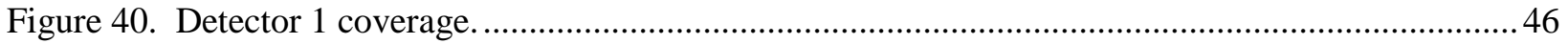

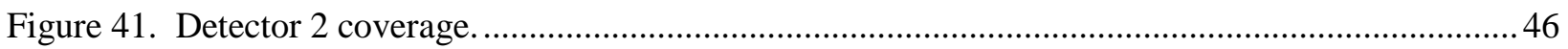

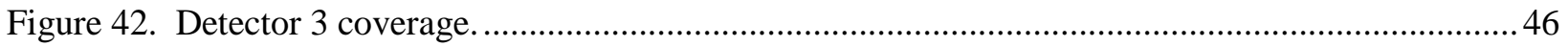

Figure 43. How many detectors are triggered for an accident at any location $(\mathrm{z}=100 \mathrm{~cm}) \ldots \ldots \ldots \ldots \ldots \ldots . . . . . . .66$ 


\section{LIST OF TABLES}

Table 1. Key results from simulations of Jezebel benchmark and CAAS fluxes (errors are at the 1- $\sigma$ level).

Table 2. Determination of the minimum accident of concern for Jezebel in a simple block building

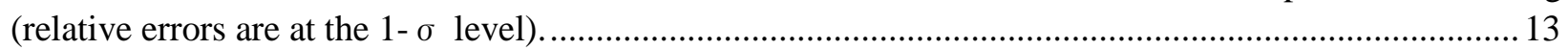

Table 3. Total leakage from Jezebel as calculated by SCALE (relative errors are at the 1- $\sigma$ level)........ 20

Table 4. Total leakage from Jezebel as calculated by MCNP (relative errors are at the $1-\sigma$ level)......... 23

Table 5. Comparison of calculated CAAS detector responses using different radiation transport codes, critical source approximations, and variance reduction (relative errors are at the $1-\sigma$ level)...................25

Table 6. Smallest detectable fission rate for the Figure 5 credible accident configuration ...................... 26

Table 7. Comparison between region tally and mesh tally dose rates at center of spherical CAAS (relative

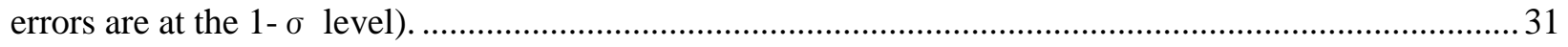

Table 8. Different approaches for CAAS detector placement studies. .................................................. 34

Table 9. Results for simple region tallies using analog and CADIS methods (highlighted doses are above the 0.150 rem alarm set point - relative errors are at the $1-\sigma$ level) ........................................................ 36

Table 10. Comparison of CAAS detector responses (rem) calculated with FW-CADIS and mesh tallies

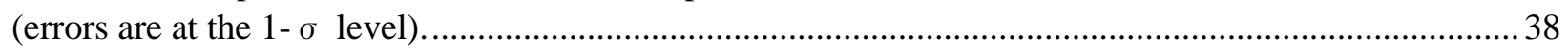

Table 11. Results of simple region tallies from forward and adjoint MCNP simulations (highlighted doses are above the 0.150 rem alarm set point - errors are at the $1-\sigma$ level).......................................... 43

Table 12. CAAS detector response (rem) calculated using adjoint Denovo (highlighted doses are above the 0.150 rem CAAS alarm set point) .................................................................................................... 45 



\section{INTRODUCTION}

This document provides guidance on performing computational analyses of Criticality Accident Alarm Systems (CAAS). A brief overview of ANSI/ANS-8.3-1997 (R2012) [1], the standard that the United States Department of Energy uses to guide the placement of CAAS detectors, is provided with a focus on the parts of the standard that are most applicable to calculating CAAS detector responses and evaluating CAAS detector coverage. This is followed by a brief discussion of how CAAS detector response calculations differ from eigenvalue calculations that criticality safety practitioners perform regularly. The remainder of the document provides computational examples of how to determine the minimum accident of concern according to ANSI/ANS-8.3-1997 (R2012), how to calculate the response of a CAAS detector due to a specific criticality accident, and how to evaluate the coverage of a CAAS detector for criticality accidents. These practical examples are solved applying SCALE 6.1 [2] and MCNP5 [3], but the methodologies can be applied to other radiation transport codes with similar capabilities, including deterministic codes.

\section{SCOPE AND LIMITATION OF THIS GUIDANCE}

The scope of this guidance document covers just the CAAS detector response calculations. There are some very important aspects of CAAS evaluations this guidance document does not address that must be addressed before any CAAS detector response calculations begin. Possibly most important is the determination of credible accidents and credible accident locations, which will vary between different applications and facilities. Therefore, it would be difficult to provide a concise guidance document on how to determine the credible accidents and credible accident locations for every application and facility. This guidance document also does not discuss a specific CAAS or the appropriate flux-to-dose-rate conversion factor for a specific CAAS. The flux-to-dose-rate conversion factor is used to convert calculated flux to the same units of dose measured by the CAAS detector. As with the credible accidents, it would be difficult to provide a concise guidance document on how to determine the appropriate flux-todose-rate conversion factor for all CAAS detectors. Ultimately, since credible accidents, credible accident locations, and flux-to-dose-rate conversion factors can vary so much between different applications and facilities, it is the criticality safety practitioner's responsibility to determine the credible accidents and locations that should be modeled and appropriate flux-to-dose-rate conversion factors that should be used in those models. These questions must be addressed before a practitioner can begin the computational analysis supporting CAAS detector placement, which is what this guidance document is focused on. The practitioner, who is responsible for performing the CAAS analysis, should work with all stakeholders (operations staff, instrumentation and controls staff, regulators, etc.) to determine the credible accidents, locations, and flux-to-dose-rate conversion factors that should be used before beginning a detailed CAAS analysis. This "buy in" from all stakeholders will hopefully prevent issues during the review of the CAAS evaluation. Finally, the potential kinetics behavior of a criticality accident and shutdown mechanisms are beyond the scope of this guidance and are not considered.

Having stated these limitations about determining credible accidents, credible accident locations, and flux-to-dose-rate conversion factors, this document does make some assumptions about credible accidents and locations, CAAS detector type, and flux-to-dose-rate conversion factors for the modeled CAAS detectors. Furthermore, since the kinetic behavior of the system is being ignored, the critical excursion and geometric and physical properties of the system (temperature, cross sections, etc.) are all treated as steady state. These assumptions are necessary in order to provide informative illustrations for this guidance document and can serve as examples to guide a practitioner in their own CAAS evaluation. However, these assumptions are not likely to meet the needs of every practitioner. 
Finally, this document does not provide any guidance on the "best" code to use for CAAS analysis. SCALE and MCNP examples are presented because these codes are widely used by the criticality community in the United States. When selecting a code for CAAS analysis, the best code will be one that has been verified and validated on the platform on which this analysis will be performed. Furthermore, a validation of the software used for this analysis may produce a bias and bias uncertainty for fixed-source simulations of the practitioner's application, which should be accounted for in the CAAS detector placement analysis if available.

\section{ANSI/ANS-8.3-1997 (R2012)}

The detection criterion stated in Section 5.6 of ANSI/ANS-8.3-1997 (R2012) can be paraphrased as follows.

A CAAS shall respond immediately to the minimum accident of concern, which may be assumed to deliver the equivalent of an absorbed dose rate in free air of $0.2 \mathrm{~Gy} / \mathrm{min}$ at 2 meters.

For the purposes of this guidance, a critical assembly of material generating a radiation dose rate of 0.2 $\mathrm{Gy} / \mathrm{min}$ in free air at 2 meters from the outer surface of the critical assembly will be the assumed minimum accident of concern. Notice that the standard does not state whether the dose rate is due to neutrons or photons, so it is reasonable to assume this refers to total dose. However, most simple detection systems, like those typically used in CAAS, only respond to neutrons or photons. The standard does not state which flux-to-dose-rate conversion factors should be used to calculate the absorbed dose in air, so unless stated otherwise, this guidance will use the air kerma factors provided by the ICRU $[4,5]$. This will allow the absorbed dose in air to be calculated in the vicinity of the CAAS detector, and will not require modeling of the actual CAAS detector. Modeling the actual CAAS detector and calculating the actual CAAS detector response is a complex task that can only be done if the detector is well characterized and the specific detector response function is known.

The standard does state that a different minimum accident of concern may be used. However, the standard does not provide details about how a minimum accident of concern may or may not vary from the provided definition. If a different minimum accident of concern is used, additional documentation is required that explains the basis. This guidance document will not discuss justifying a different minimum accident of concern, but the principles presented here can be applied to any minimum accident of concern.

Section 5.8 of ANSI/ANS-8.3-1997 (R2012) discusses the spacing and placement of the CAAS detectors. The standard states,

"The location and spacing of detectors should be chosen to minimize the effect of shielding by massive equipment or materials.”

Evaluation of the coverage of a CAAS detector can most accurately be performed via a radiation transport calculation. The results of such an evaluation will provide direct insight into the effects of location and spacing of CAAS detectors. The accuracy that can be obtained in a CAAS evaluation is dependent upon the level of detail included in the model and on the capabilities of the radiation transport code. The results of such an evaluation will directly address Section 5.8 of ANSI/ANS-8.3-1997 (R2012) in a straightforward and conclusive manner. 


\section{BASICS OF TRANSPORT CALCULATIONS REQUIRED TO PERFORM CAAS ANALYSIS}

This section discusses fundamental differences between CAAS detector response calculations and system eigenvalue $\left(\mathrm{k}_{\text {eff }}\right)$ calculations. The primary difference in setting up an input file for a CAAS calculation is that the CAAS detector response calculation will require a set of flux-to-dose-rate conversion factors, which represent the energy dependence of the detector response. Additionally, some representation of the spatial dependence of the detector response may be useful. The final requirement, which is not needed by every radiation transport code, is the description of one or more fixed sources, including the source strength, that are used to calculate the CAAS detector response. Whether or not the practitioner needs to provide a fixed source can be determined by answering the question, "Can the eigenvalue calculation provide the detector dose rates as well as the $k_{\text {eff }} v a l u e$ ?" If the answer to this question is yes, then a fixed source calculation is not required. If a Monte Carlo code is being used, and variance reduction is required to calculate the CAAS detector response, then performing a fixed-source calculation will likely simplify the task of calculating the CAAS detector response. These different requirements can be illustrated by comparison of the features of some specific radiation transport codes.

When deterministic codes, such as XSDRNPM [2,6], PARTISN [7], or Denovo [2,8], are used to calculate the system eigenvalue, they also calculate fluxes throughout the entire geometry. These fluxes can be convolved with the flux-to-dose-rate conversion factors as part of the eigenvalue calculation, so a description of the fixed source is not required. In fact, the outer iterations of these deterministic calculations are calculating the fission source and updating the fluxes in the entire geometry due to this source. This is true for all deterministic radiation transport codes. One only needs to be sure that the fission source is converged and that the neutron and photon flux solutions are converged throughout the entire geometry.

The description of the fixed source may be required with a Monte Carlo calculation of the CAAS detector response. For example, KENO [2,9] cannot easily calculate a CAAS detector response during its eigenvalue calculation. In fact, KENO does not perform any photon transport, so it cannot calculate a photon detector response. Therefore, to perform this sort of analysis using three-dimensional Monte Carlo codes in SCALE, KENO must be used to calculate the fission source, which is then used as a fixed source by Monaco $[2,10]$ along with any necessary variance reduction to calculate the CAAS detector response. On the other hand, MCNP can easily calculate flux tallies during an eigenvalue calculation, including photon tallies. However, if variance reduction is required to calculate the CAAS detector response, which will often be the case for CAAS applications that involve large amounts of shielding, the variance reduction techniques that are applied must not have a negative effect on the convergence of the eigenvalue calculation. Similar to deterministic calculations, the fission source must be converged in these Monte Carlo calculations, and the flux at the location of the CAAS detectors must also be well converged.

The primary difference between the requirements to perform CAAS analysis with different radiation transport codes is whether or not the practitioner needs to provide a fixed-source. To illustrate how this difference affects the computational methodology, a flow chart that shows how a CAAS detector response is calculated with and without a fixed source is shown in Figure 1 and Figure 2, respectively. As noted in the introduction, this guidance would provide examples that illustrate the use of SCALE 6.1 and MCNP5; so, computational flow charts are laid out using these codes. Figure 1 focuses on the use of a fixed source and uses the SCALE code system, but the same methodology could be applied with any radiation transport code with a fixed source, including MCNP. Figure 2 focuses on not using a fixed source, which works for any code that can use the flux-to-dose-rate conversion factors as part of an eigenvalue calculation (SCALE cannot do this for a photon response). In Figure 1 and Figure 2, the rectangles represent input to a radiation transport code, diamonds represent the actual calculations, ovals represent output from a radiation transport code, and MAOC is the minimum accident of concern. At this point in 


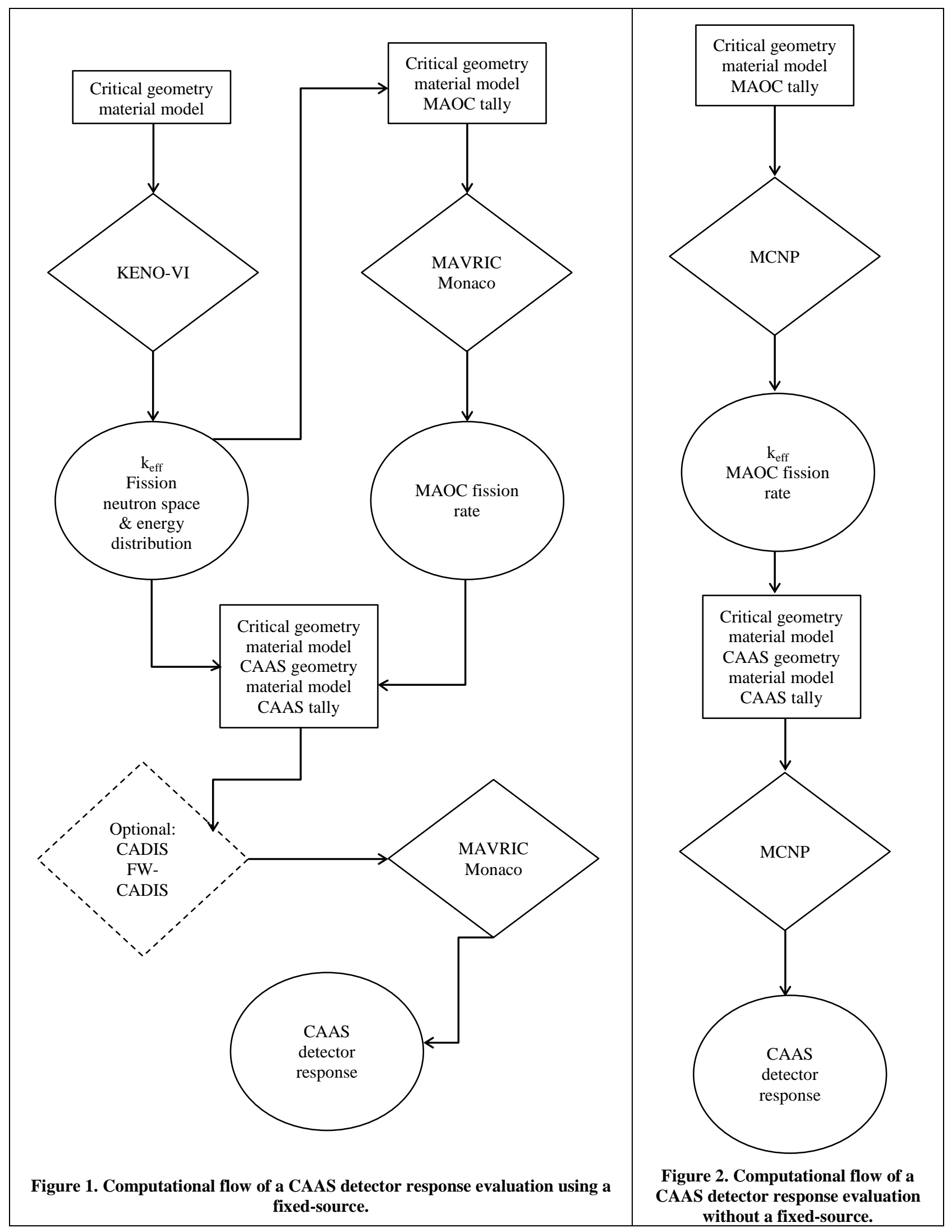


the discussion of computational analysis of CAAS, all the details in the computational flow charts may not be understood by the reader. Keep referring back to these figures as you proceed through Sections 4 7 , and the meaning of the details will become clearer.

The following subsections provide a very simple example of how an input file for a standard eigenvalue calculation is modified to calculate a CAAS detector response using MCNP and SCALE. The base eigenvalue calculation used in the following subsections is the Jezebel benchmark (simple sphere of $\mathrm{Pu}$ ), whose description is available from the International Criticality Safety Benchmark Evaluation Project (ICSBEP) as PU-MET-FAST-001 [11]. For this example, the geometry of the Jezebel benchmark has been modified to include a CAAS detector, which is represented by a sphere of air the same diameter as Jezebel $(\sim 13 \mathrm{~cm})$, centered 1.0 meter from the center of Jezebel (i.e. two spheres a meter apart). Full input files for these calculations are provided in Appendix A.

\subsection{SCALE 6.1}

The SCALE input file to calculate the system eigenvalue for the Jezebel benchmark with multi-group KENO-VI is included in Appendix A.1. To calculate the CAAS detector response with SCALE, all three additional requirements mentioned in Section 4 are required: flux-to-dose-rate conversion factors, a geometric model representing the CAAS detector (with as much or little detail as desired), and a description of the fission source as a fixed source. For the purposes of this initial example, the flux-todose-rate conversion factors will be ignored, and the flux per source neutron, rather than the dose rate, will be calculated. The additional material and geometry cards needed to describe this detector are in the full SCALE input file for this modified geometry in Appendix A.2. As noted in Section 4, the fixed source is needed because KENO cannot easily be used to calculate fluxes or responses in a specific region of the geometry. The CAAS analysis capability in SCALE was developed to address this specific type of problem [12]. The SCALE CAAS analysis capability consists of the following steps:

- Run KENO to calculate the spatial- and energy-dependent distributions of the neutrons created by fission, which are saved as a mesh tally file (this is the KENO-VI calculation in the top left of Figure $1)$.

- The neutron mesh tally file is converted to a spatial- and energy-dependent neutron fixed source file.

- The SCALE MAVRIC $[2,10]$ sequence is used to run Monaco, with the option of using the automated variance reduction methodologies, to calculate the CAAS detector response (this is the MAVRIC/Monaco calculation at the bottom of Figure 1).

The input file shown in Appendix A.2 includes all of the input required to perform the entire sequence of calculations that make up the SCALE CAAS analysis capability. Below is a brief discussion of some of the lines of input in Appendix A.2 that are unique to and important for the SCALE CAAS analysis capability.

First, a parameter telling KENO that a spatial- and energy-dependent tally of fission neutrons is needed must be set in the parameters block in the KENO input. This parameter (CDS) must be set to "yes" as shown below:

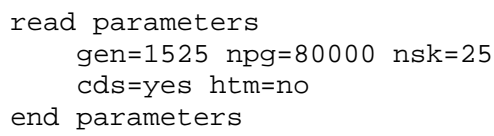

Next, a mesh needs to be defined specifying the spatial domain over which KENO will tally the fission distribution. The input segment below illustrates how this is entered into KENO for this problem: 


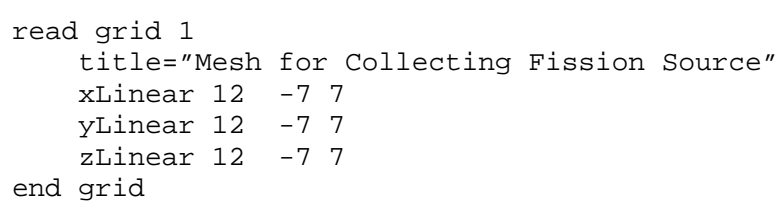

These lines of input create a mesh in the global unit coordinate system that extends from $-7 \mathrm{~cm}$ to $7 \mathrm{~cm}$ in all three directions, with 12 mesh cells between $-7 \mathrm{~cm}$ and $7 \mathrm{~cm}$ in all three dimensions. The mesh will have a total of $1728\left(12^{3}\right)$ cells, which are enough cells such that the cell sizes are not very large compared to the mean-free-path of neutrons in the system. KENO saves this mesh tally to a file named "fissionSource.3dmap." The mesh does not need to cover the entire KENO geometry, but it must cover all of the regions of fissionable material modeled in the following MAVRIC/Monaco fixed-source calculation (thus $-7 \mathrm{~cm}$ to $7 \mathrm{~cm}$ in each direction). Additionally, the geometry in the KENO calculation does not have to match the geometry of the MAVRIC/Monaco calculation. The MAVRIC/Monaco calculation will often include geometry details, omitted from the KENO source calculation, that are important for the fixed-source radiation transport calculation of the CAAS response. For example, the KENO model may include only the critical assembly, while the MAVRIC/Monaco model will also include the building and equipment surrounding the critical assembly.

The energy group structure of the KENO fission neutron distribution tally will be determined by the group structure of the cross sections used for the KENO calculation. This cross-section library does not have to be the same as the cross-section library used in the subsequent MAVRIC/Monaco fixed-source calculation. This method to define the energy-group structure of the fission neutron distribution tally requires that the KENO source calculation use multi-group cross sections.

The mesh tally calculated by KENO is converted to a mesh source for Monaco via the utility MT2MSM

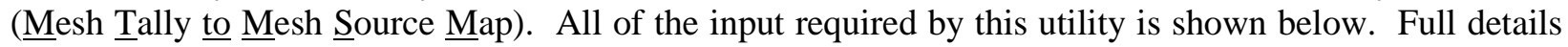
about this utility are available in the appendices of the SCALE MAVRIC manual [2]. The default file name for the fission tally saved by KENO is "fissionSource.3dmap." Running the input below for MT2MSM saves the Monaco fission source in the file "fissionSource.msm." The sequence of integers in the input $(1,-1$, and 1$)$ signals the code to use the first family of data, convert all energy groups, and that the particles are neutrons, respectively.

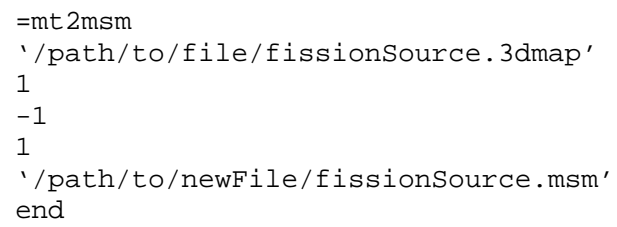

The composition and geometry blocks used in the KENO-VI input can be copied directly into the Monaco input. As noted above, the Monaco geometry can contain more detail than the KENO geometry, so it is reasonable that the Monaco geometry may be much larger than the KENO geometry (e.g., modeling an entire facility versus just a critical assembly).

The fixed-source is entered into Monaco (after the material and geometry specifications) using the following lines of input: 


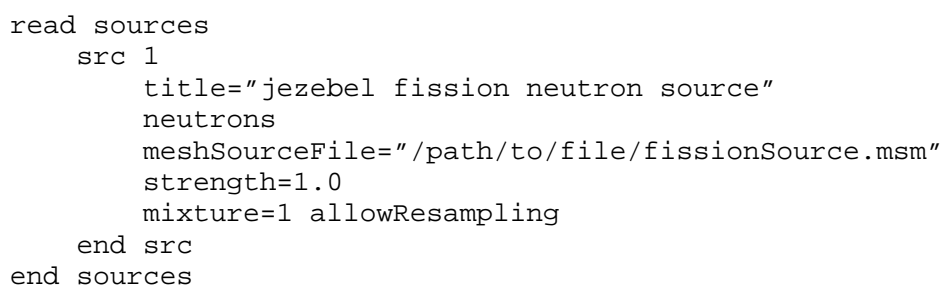

The spatial and energy dependence of the fission source is read into Monaco by pointing the input variable "meshSourceFile" to the file created by the MT2MSM utility ("fissionSource.msm"). For mesh sources the origin of the KENO and Monaco global units are assumed to be in the same location. However, the "origin" keyword can be used in the sources block to translate the location of the mesh source within the Monaco geometry. Notice that the source strength is set using the "strength" keyword, so the source strength is set to 1 fission neutron per second, so the flux tally results will be calculated on a basis of per fission neutron per second. Although not used in this example, it is also possible to specify the number of fission events using the "fissions" keyword. In that case, it is important that the file named "kenoNuBar.txt," created by KENO when the parameter CDS equals “yes," is in the SCALE temporary directory when Monaco is executed. If KENO and Monaco are not run in a single SCALE calculation, the "kenoNuBar.txt" file is returned by KENO with the SCALE input filename appended to the beginning, i.e., the keno input name.in returns name.kenoNuBar.txt. However, when this file is moved into the Monaco temporary directory it must be named "kenoNuBar.txt." Monaco reads $\bar{v}$ from this file to convert the number of fission events to the total number of fission neutrons. The parameter "mixture" is set equal to 1 to tell Monaco to only start particles whose positions are sampled inside of a region containing mixture 1. This is needed because the fissile material is a sphere and the fission source was saved on a Cartesian mesh in KENO. Without this limitation on mixture (or a similar limitation on unit and region), source particles could be started outside the plutonium sphere but inside the Cartesian mesh. Furthermore, it is possible that only a very small amount of the plutonium sphere may be included in some of the outer mesh cells; so, occasionally, Monaco may not be able to locate material 1 within a given mesh cell. Therefore, the keyword "allowResampling" is added, which tells the code to resample the Cartesian mesh if mixture 1 has not been found after a number of samples within a specific mesh cell instead of stopping the calculation with an error due to sampling the source particle position. This sort of resampling can introduce a bias in the computational results, so it should be used with caution. Whenever a particle is thrown away and a new source particle is sampled, a warning message is printed in the Monaco output. The number of these warning messages should represent a very small fraction of the total number of sampled sources particles $(<<1 \%)$. Otherwise, there is likely a problem with the source and the results may be biased. For this problem, 1203 source particles were resampled out of $10^{8}$, which is $\sim 0.001 \%$. Note that any KENO mesh cell that does not contain fissionable material will never be sampled by Monaco, so all mesh cells initially sampled by Monaco contain some fissionable material (even though it may be very little).

The flux tally is entered into Monaco with these lines of input:

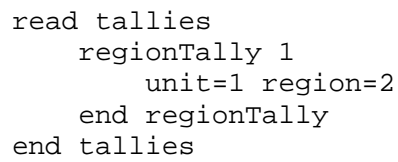

Tally 1 will calculate the flux in region 2 of unit 1 . The regions in a unit are numbered sequentially based on all the media, hole, and array cards in that unit. In this SCALE input, the detector is defined by the second media card in global unit 1 . Thus, it is treated as region 2 for the purpose of a region tally.

The final important input parameters are in the Monaco parameters block. In this block, the "noFissions" keyword must be included because the neutron production due to fission has already been accounted for 
in the KENO calculation that was used to create the fixed source for Monaco. Note that if the "noFissions" keyword is not included in a Monaco calculation for a system whose eigenvalue is close to or greater than 1.0, neutrons will be created and added to the Monaco particle bank faster than they are removed. This will cause single histories to last for a very long period of time or until the computer's entire memory has been used. If photon fluxes and/or responses are not needed, use the "noSecondaries" keyword in the Monaco parameters block. The source input shown above includes only a neutron source, thus in order to accurately model photon production do not use the "noSecondaries" keyword [12].

While this SCALE CAAS calculation requires running KENO and Monaco in two separate calculations, this is a great advantage if variance reduction is applied to calculate the CAAS detector response. Since Monaco is a fixed-source Monte Carlo code, the source and particle transport can be biased without regard to the source convergence in the critical assembly required by an eigenvalue calculation. The MAVRIC/Monaco input file in Appendix A.2 uses standard biasing techniques available in Monaco, but the automated variance reduction techniques CADIS and FW-CADIS [10,13] available as part of the MAVRIC sequence are available if needed. Additionally, the CADIS and FW-CADIS techniques can be applied with MCNP via the code ADVANTG [14].

It is beyond the scope of this guidance document to discuss the CADIS and FW-CADIS automated variance reduction techniques in detail. A full description can be found in References 10, 13, and 14. However, as these variance reduction techniques are referred to and applied in this guidance document, a short introduction is warranted. CADIS is a method that generates weight windows and a consistent biased source that optimize a Monte Carlo simulation to calculate one specific tally. CADIS calculations use an adjoint deterministic calculation (importance map calculation) to create these weight windows and a consistent biased source distribution. FW-CADIS is a method that generates weight windows and a consistent biased source to optimize a Monte Carlo simulation with multiple tallies. This method attempts to provide uniform uncertainties for all of the tallies in the problem, which is particularly useful for a single tally location using multiple sets of flux-to-dose rate conversion factors or multiple tally locations using any number of flux-to-dose-rate conversion factors (e.g. a large mesh tally that covers high and low flux areas of a single problem). FW-CADIS calculations use two deterministic calculations (one forward and one adjoint) to create these weight windows and consistent biased source distribution.

\subsection{MCNP5}

The MCNP input file to calculate the system eigenvalue for the Jezebel benchmark is included in Appendix A.3. To calculate the CAAS detector response with MCNP, only the flux-to-dose-rate conversion factors and the geometric model representing the CAAS detector are required in addition to the input in Appendix A.3. Like the SCALE calculation, for the purposes of this initial example, the fluxto-dose-rate conversion factors will be ignored. The additional material and geometry cards needed to describe the geometric model of the CAAS detector are in the full MCNP input file for this modified geometry in Appendix A.4. Below is a brief discussion of some of the lines of input in Appendix A.4 that are unique to and important for the MCNP evaluation of the CAAS detector response.

The tallies required to calculate the flux in the CAAS detector (cell 50) are as follows:

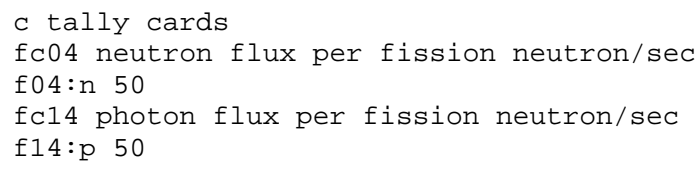

One can see that this is much simpler than what is required by SCALE, because this tally can be included directly in the MCNP eigenvalue calculation (this is the MCNP calculation at the bottom of Figure 2). However, if variance reduction is required to calculate the CAAS detector response, MCNP does not offer any automated variance reduction techniques, and source biasing is not an option with eigenvalue 
calculations. Note that variance reduction techniques such as weight windows, if not applied correctly, can have a negative impact on the neutron transport in the fuel region of this problem, which could result in an incorrect eigenvalue and an unconverged fission source. The process in SCALE known as the CAAS analysis capability can be duplicated with MCNP, but there is no automated utility that currently exists to convert an MCNP mesh tally created during an eigenvalue calculation to an MCNP fixed source.

\subsection{SUMMARY}

To complete the discussion about the input features needed by SCALE and MCNP to evaluate a CAAS detector response, it is not imperative to discuss the computational results produced by the input files in Appendices A.1-A.4. However, for completeness, these results are presented here to show that both codes can produce similar results. Additionally, this offers the opportunity to discuss some of the differences between photon results produced by SCALE and MCNP. Table 1 provides the key results produced by SCALE and MCNP with the input files in Appendices A.1-A.4.

Table 1. Key results from simulations of Jezebel benchmark and CAAS fluxes (errors are at the 1- $\sigma$ level).

\begin{tabular}{|c|c|c|}
\hline Result & SCALE & MCNP \\
\hline Jezebel $\mathrm{k}_{\text {eff }}$ & $0.997820 \pm 0.000083$ & $0.99952 \pm 0.00005$ \\
\hline CAAS Total Neutron Flux & $0.999271 \pm 0.000084^{\dagger}$ & $5.3310 \mathrm{E}-06 \pm 0.33 \%$ \\
\hline CAAS Total Photon Flux & \\
\hline & $5.3854 \mathrm{E}-06 \pm 0.37 \%$ & $1.5870 \mathrm{E}-06 \pm 0.98 \%$ \\
\hline \\
${ }^{\dagger}$ continuous energy & $1.4923 \mathrm{E}-06 \pm 0.86 \%$ & \\
${ }^{\ddagger}$ particle flux per fission neutron per second
\end{tabular}

All of the results were calculated using ENDF/B-VII.0 cross sections. The SCALE results used multigroup cross sections, except the results marked continuous energy, and the MCNP calculations all used continuous-energy cross sections. The agreement between the eigenvalues calculated using continuousenergy cross-section data is good, as is the agreement between the total neutron fluxes. The SCALE total neutron flux is about $1 \%$ greater than the MCNP value. The MCNP total photon flux is about $6 \%$ greater than the SCALE value. Even with this difference, the agreement between SCALE and MCNP for the photon fluxes is considered good. This is expected because MCNP models additional photon physics and includes photon cross-section data down to lower energies than SCALE. The photon cutoff energies due to the cross-section libraries are $1 \mathrm{keV}$ in MCNP and $10 \mathrm{keV}$ in SCALE. Additionally, MCNP includes a thick-target bremsstrahlung model that produces low energy photons due to the deceleration of electrons that are produced in photon interactions, and MCNP will Doppler energy broaden photon cross sections. SCALE does not model these phenomena. This difference should be kept in mind when comparing MCNP and SCALE photon results and considered when using SCALE for CAAS analysis. The magnitude of this difference is problem dependent, and it is beyond the scope of this guidance document to quantify this difference for all situations. Past experience has shown that this difference can be very small (less than 1\%) up to a few 10s of percent, but usually the SCALE photon flux is less than the MCNP photon flux (flux, not necessarily dose).

\section{DETERMINING THE MINIMUM ACCIDENT OF CONCERN}

Once a credible accident and accident location have been established, the minimum accident of concern needs to be determined. The assumption stated in Section 3, which is based on the guidance in ANSI/ANS-8.3-1997 (R2012), sets the minimum accident of concern in this document to be a criticality accident that produces a dose rate of $0.2 \mathrm{~Gy} / \mathrm{min}$ in free air two meters from the outer surface of the critical assembly. A practitioner will quickly realize that this definition of the minimum accident of concern is not overly prescriptive. It is left to the practitioner to work with the concerned stakeholders to determine the details that best suit each specific application. Different critical configurations could meet 
this criterion of a minimum accident of concern with dramatically different fission rates, so establishing a minimum accident of concern based on a set number of fission events or fission rate will not equally address the risk to personnel due to exposure to radiation. Therefore, the practitioner needs to determine the fission rate that produces the minimum accident of concern for their specific credible accident. This determination can be done in either of two different ways.

First, a measurement could be performed to determine the fission rate when a detector reported that the dose rate in air two meters from the critical assembly reached $0.2 \mathrm{~Gy} / \mathrm{min}$. However, this is likely to be an expensive proposition and not likely to be very practicable. The second option is to calculate the fission rate required to produce a dose rate of $0.2 \mathrm{~Gy} / \mathrm{min}$ in air two meters from the critical assembly. The drawback to this second option is that the practitioner needs to use an appropriate set of flux-to-doserate conversion factors to calculate the neutron and photon flux that produces a measurement of 0.2 $\mathrm{Gy} / \mathrm{min}$ in air two meters from the critical assembly.

This document provides guidance on how to calculate CAAS detector responses, so the second option to determine the minimum accident of concern, i.e. calculate it, will be used. Once again, this option requires the selection of an appropriate set of flux-to-dose-rate conversion factors. To make an appropriate selection, consider the quantity that needs to be calculated (e.g., absorbed dose or dose equivalent) and the sensitivity of the actual CAAS detector (e.g., does the detector respond to neutrons and/or photons and at what energies). Selection of a set of flux-to-dose-rate conversion factors should be based on input from all stakeholders involved in the CAAS analysis. In this document, unless stated otherwise, the air kerma factors provided by the ICRU will be used to convert between calculated flux and calculated dose rate. The ICRU air kerma factors are just one of many available flux-to-dose-rate conversion factors, and are likely not appropriate for all applications. For a little historical perspective, the Criticality Safety Slide Rule [15] used the Henderson conversion factors, which are appropriate because the detector for that application is a human.

The following example demonstrates how to calculate the fission rate that produces the minimum accident of concern using the ICRU air kerma factors. First, a simple credible accident needs to be introduced. The credible accident consists of Jezebel (PU-MET-FAST-001) in a simple block building, which is $1200 \mathrm{~cm}$ long, $600 \mathrm{~cm}$ wide, and $300 \mathrm{~cm}$ high above the ground. The exterior and interior walls are all made of a double layer of typical concrete blocks (total of $40 \mathrm{~cm}$ thick). The floor is made of poured concrete, extending $60 \mathrm{~cm}$ into the ground. The roof and the exterior door $(120 \mathrm{~cm}$ wide and 210 $\mathrm{cm}$ tall) are made of $0.3175 \mathrm{~cm}$ thick steel. The center of Jezebel is $100 \mathrm{~cm}$ above the concrete floor of this building. The building and Jezebel (red sphere in left room) can be seen in Figure 3 and Figure 4. Also visible in Figure 4 is part of a spherical shell (purple) with an inner radius that is two meters from the outer surface of Jezebel. This spherical shell marks where the dose rate will be calculated to determine the minimum accident of concern. To determine the minimum accident of concern, the dose rate per fission event will be calculated on the spherical shell, which will then be used to determine the fission rate required to reach $0.2 \mathrm{~Gy} / \mathrm{min}$. Since the top and the bottom of the purple spherical shell extend above the roof and into the concrete floor, the top and bottom have been cut off. The dose rate per fission will only be considered for locations that have a direct line of sight (unshielded) to the critical assembly. The building surrounding Jezebel will also contribute to the calculated dose rate per fission due to reflection from the floor and concrete walls. In this example, modeling Jezebel within the concrete building is not especially important. However, if the credible accident location placed Jezebel close to a reflector, this would need to be considered. The following subsections provide details about the input that must be added to the Jezebel models in Appendices A.1 and A.3 to calculate the minimum accident of concern. 


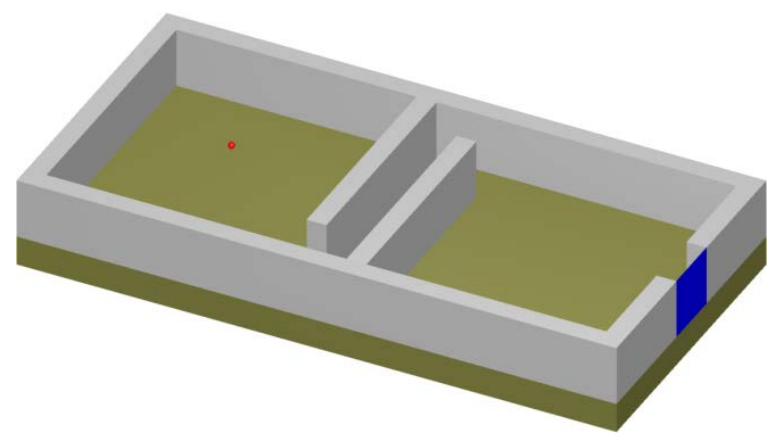

Figure 3. Block building with Jezebel, top half removed.

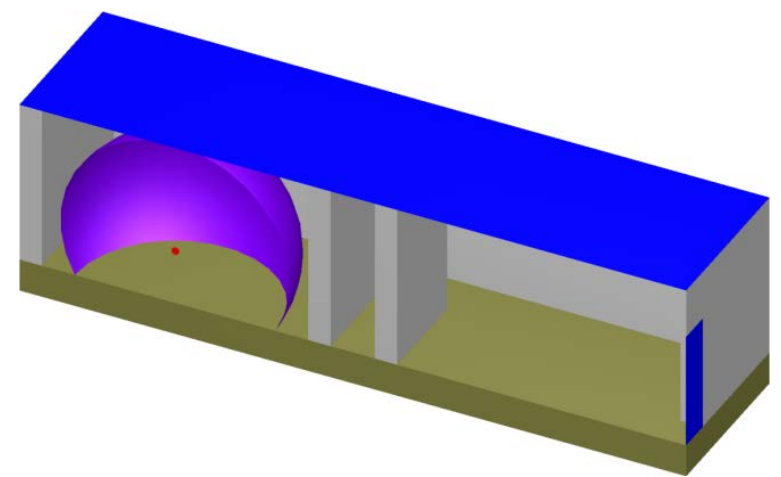

Figure 4. Block building with Jezebel and two-meter tally sphere, front half removed.

\subsection{SCALE 6.1}

To calculate the minimum accident of concern with SCALE, the SCALE CAAS analysis capability discussed in Section 4.1 is applied. The KENO-VI calculation at the top left of Figure 1 is run to determine the spatial- and energy-dependent distributions of the neutrons created by fission, which is input into the MAVRIC/Monaco calculation at the top right of Figure 1 to calculate the fission rate that produces the minimum accident of concern. The complete SCALE input for this sequence of calculations is included in Appendix A.5. The Jezebel experiment KENO input is modified to include the materials and geometry of the block building, to account for the new location of Jezebel in the mesh grid input (Jezebel's center is no longer at the origin), and to set the parameter CDS to yes. KENO start data have been added to accelerate the process of starting neutrons in the first generation, but this is not required. The input for the utility MT2MSM is unchanged. Similar to the KENO input, the Monaco input needs to be modified to include the materials and geometry of the block building. The spherical shell that will be used to tally dose two meters from Jezebel must also be added to the Monaco geometry. The media card describing the spherical shell in the Monaco geometry must include the volume for Monaco to calculate the flux correctly. For this example, the volume of the spherical shell was calculated using KENO-3D [2].

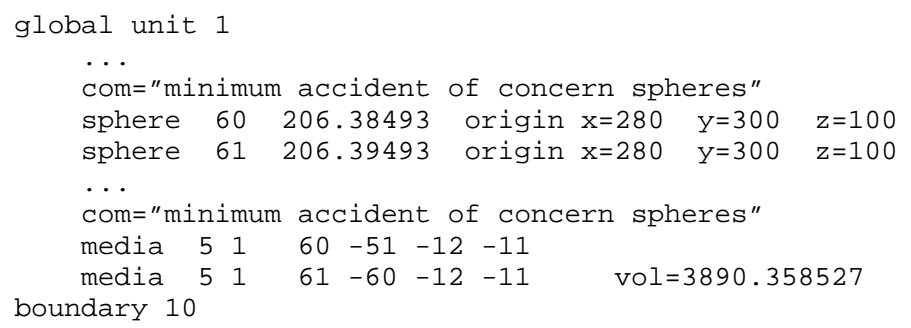

Unlike the calculations in Section 4.1, dose rate is calculated, so flux-to-dose-rate conversion factors need to be provided in the input. The ICRU air kerma factors available in SCALE need to be requested in the definitions block. Note that the flux-to-dose-rate conversion factors have units of Gy/hr per unit flux.

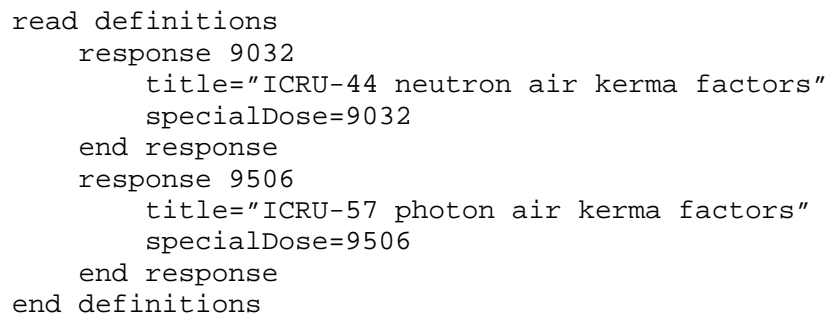


Next the source is entered in a manner very similar to that in Section 4.1. However, now the number of fissions per second is entered via the "fissions" keyword instead of the number of source neutrons. Again, $\bar{v}$ is read from the file "kenoNuBar.txt" created by the KENO calculation.

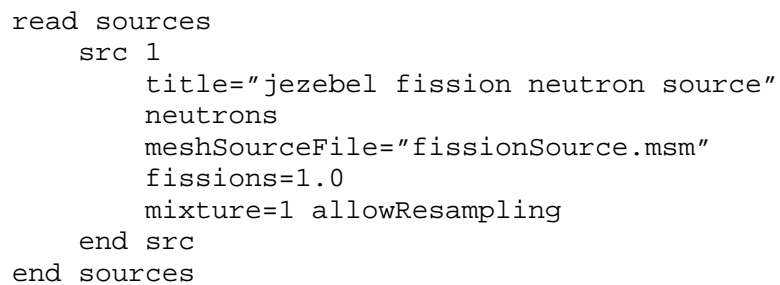

A region tally must be created for the spherical shell that is two meters from Jezebel, so a tally similar to that in Section 4.1 is needed. However, this tally needs information about flux-to-dose-rate conversion factors because more than flux needs to be calculated. The response identifiers (IDs) assigned to the air kerma factors in the definitions block are used with this tally. Also note that the tally multiplier is equal to $1 / 60$ to convert $\mathrm{Gy} / \mathrm{hr}$, the default units of the kerma factors, to $\mathrm{Gy} / \mathrm{min}$. Alternatively, this multiplier could have been applied to the flux-to-dose-rate conversion factors in the definition block, which would be more convenient if they were used in more than one tally.

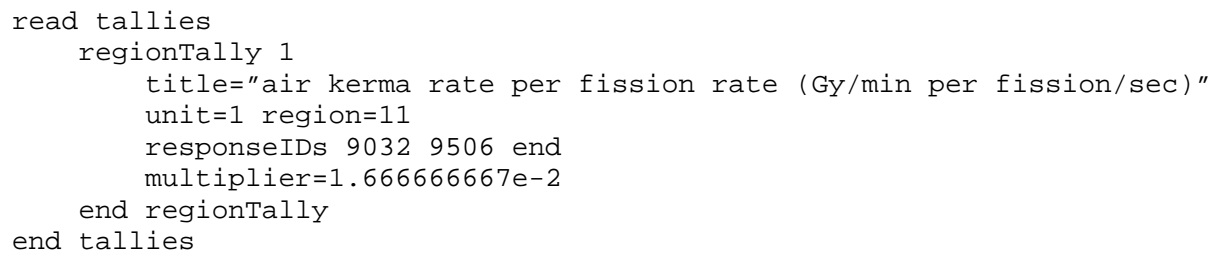

Finally, the "noFissions" keyword must be used, in the Monaco parameters block, with this critical system to avoid generation of fission neutrons already included in the source, and the "noSecondaries" keyword is not used because photon air kerma rates are being calculated in addition to neutron air kerma rates.

\subsection{MCNP5}

Determining the fission rate that produces the minimum accident of concern with MCNP can be less complex relative to SCALE (i.e., similar to how the calculation in Section 4.2 is less complex). This is because the tally for the minimum accident of concern can be put directly into the MCNP eigenvalue calculation, which is represented by the MCNP calculation at the top of Figure 2. Similar to the SCALE calculation of the minimum accident of concern, the MCNP model of the Jezebel benchmark is modified to include the materials and geometry of the block building to calculate the dose rate per fission rate. The complete input file that shows of all the geometry changes is available in Appendix A.6. Also similar to the SCALE calculation, the MCNP input needs the volume of the spherical shell and the flux-to-dose-rate conversion factors. The volume input is required by MCNP because it cannot calculate the volume of the truncated spherical shell due to its asymmetric shape. This volume could be entered on the SD card for each individual tally or on the cell volume card. Below are the lines of input that enter this volume on the cell volume cards.

c data cards

mode $n \mathrm{p}$

c geometry cards

vol 9j 3890.358527 j

Next are several lines of input that show how the flux-to-dose-rate conversion factors are entered into MCNP. Since these flux-to-dose-rate conversion factors are not built into MCNP, they are entered as an 
energy multiplier (E and EM cards). On the lines below, the full flux-to-dose-rate conversion factors are not shown for brevity, but the full details are in Appendix A.6.

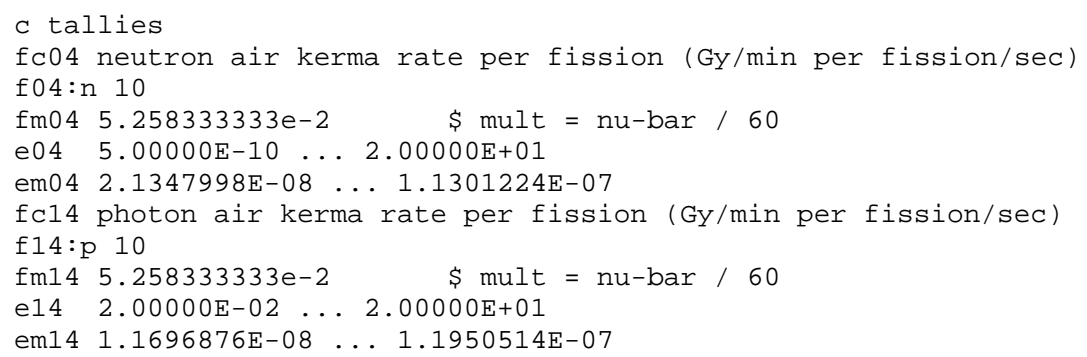

A tally multiplier (FM card) has also been added to each of the tallies shown above. For both tallies, the multiplier is equal to $\bar{v}$ divided by 60 . Similar to the SCALE tallies, the multiplier includes 1/60 to convert from $\mathrm{Gy} / \mathrm{hr}$ to $\mathrm{Gy} / \mathrm{min}$. The value $\bar{v}$ is included so the results will be per fission event instead of per fission neutron. Using the Monaco source keyword "fissions" does this automatically in the SCALE calculation. However, there is no analog to this Monaco keyword in the MCNP source, so the value of $\bar{v}$ is entered by hand via the tally multiplier. Note that the value of $\bar{v}$ used in the MCNP calculation corresponds to the value reported by the MCNP eigenvalue calculation instead of the value reported by KENO, which are slightly different.

\subsection{SUMMARY}

The results of the SCALE and MCNP calculations are the neutron and photon air kerma rates in the thin spherical shell two meters from the outside surface of Jezebel. The results of these two Monte Carlo codes (and practically all others) are reported on a per source particle basis, e.g., per fission neutron. In order for the Monte Carlo tallies to produce flux results (particle/ $\mathrm{cm}^{2} / \mathrm{sec}$ ), the results must be reported on a basis of per source particle per second, so the source strength must have units of fission neutron/sec. Since these results have been multiplied by $\bar{v}$ (assume units of neutrons/fission/sec), the tallies have returned the dose rate due to a single fission event per second, not just a single fission neutron per second. Below in Table 2 are the neutron and photon air kerma rates calculated by SCALE and MCNP and the fission rate that produces the minimum accident of concern for this system calculated by these codes. The fission rate that produces the minimum accident of concern is calculated using Eq. (1),

$$
M A O C=\frac{D_{M A O C}}{D_{n}+D_{\gamma}}
$$

where $M A O C$ is the fission rate that produces the minimum accident of concern, $D_{M A O C}$ is the dose rate defined as the minimum accident of concern $\left(0.2 \mathrm{~Gy} / \mathrm{min}\right.$ at two meters), and $D_{n}$ and $D_{\gamma}$ are the neutron and photon dose rate per fission rate at two meters, respectively. The neutron and photon dose rate per fission rate in this example are the air kerma rates provided in Table 2.

Table 2. Determination of the minimum accident of concern for Jezebel in a simple block building (relative errors are at the 1- $\sigma$ level).

\begin{tabular}{|c|c|c|}
\hline Result & SCALE & MCNP \\
\hline Neutron air kerma (Gy/min per fission/sec) & $1.83133 \mathrm{E}-15 \pm 0.074 \%$ & $1.8352 \mathrm{E}-15 \pm 0.02 \%$ \\
\hline Photon air kerma (Gy/min per fission/sec) & $5.85128 \mathrm{E}-16 \pm 0.183 \%$ & $6.6624 \mathrm{E}-16 \pm 0.05 \%$ \\
\hline Minimum Accident of Concern (fissions/sec) & $8.2766 \mathrm{E}+13$ & $7.9954 \mathrm{E}+13$ \\
\hline
\end{tabular}

As in Table 1, the SCALE and MCNP neutron results in Table 2 agree very well. The MCNP photon air kerma rate is higher ( $14 \%$ ) due to the lower photon cutoff energy and the additional low energy physics modeling bremsstrahlung. This difference in the photon air kerma rate causes a difference of about 3.5\% 
in the fission rates for the SCALE and MCNP minimum accident of concern. The SCALE minimum accident of concern is higher because the SCALE photon air kerma is underestimated.

\section{POTENTIAL SIMPLIFICATION OF THE MINIMUM ACCIDENT OF CONCERN}

In Sections 4 and 5, fixed-source simulations that calculate the flux at a detector and the minimum accident of concern are discussed. Also mentioned is the fact that variance reduction techniques may be required to calculate the CAAS detector response due to a critical assembly if a Monte Carlo code is being used. These variance reduction techniques are more easily applied to a fixed-source Monte Carlo calculation. Thus far, the only method described to model a critical assembly as a fixed-source simulation has been the SCALE CAAS analysis capability, which ultimately uses the Monaco fixed-source Monte Carlo code to calculate the detector response. A simple way to model the criticality as a fixed-source in MCNP is to reduce the criticality accident to a point source. This section will describe, at a high level, a simple method that can be used with any radiation transport code to model a critical assembly as a fixed source.

The physical description of the minimum accident of concern (spatial and energy dependence) may be simplified by treating the minimum accident of concern as a point source. This simplification removes the spatial dependence from the minimum accident of concern and allows the practitioner to remove the critical assembly from the detector response calculation (shielding calculation). This is a common simplification made in shielding calculations and makes it easy to model the criticality accident as a fixed-source in practically any radiation transport code. However, it is important to consider the consequences associated with this simplification, and to only make this simplification when the impacts of the simplification are negligible. Below are two key conditions:

- The detector geometry should be a significant distance away from the source geometry.

- Once radiation exits the source geometry, that radiation does not interact with the source geometry again before arriving at the detector geometry.

The first of these conditions can easily be evaluated by considering the distance between different points within the source and detector geometries. A good rule of thumb to remember when considering the first condition is that the distance between the detector and the source geometries should be at least 7 to 10 times the largest pertinent dimension of the source geometry. If this rule of thumb has been satisfied, then further consideration of the source/detector geometry simplification is warranted. For the accident described in Section 5 and as shown in Figure 3 and Figure 4, the largest pertinent dimension of Jezebel is the diameter, which is $12.76986 \mathrm{~cm}$. Therefore, the first condition above should be further evaluated if the detector is more than $91 \mathrm{~cm}$ from Jezebel.

Unlike the first condition, the second condition listed above does not have a simple straightforward mathematical way to evaluate. To evaluate the second condition, a more intuitive method is applied by answering this question: If a particle exits (leaks from) the source geometry, what is the probability that radiation, or any secondary radiation it produces, will interact with the source geometry again before it can reach the detector geometry? To satisfy the second condition, the answer to this question must be that the probability is small. The probability that radiation exiting Jezebel in Figure 3 and Figure 4 will interact with Jezebel again is small because the room Jezebel is located in is large (compared to Jezebel) and there are no large and dense "reflecting objects" near Jezebel (less than 10s of cm from Jezebel). If Jezebel were moved into the corner of a room or sat on the floor, this condition would likely no longer be satisfied. This is the case because a significant fraction of the radiation exiting Jezebel would scatter in the nearby walls or floor and reenter Jezebel. Therefore, any additional interactions in Jezebel could not be accounted for due to the fact that the critical assembly has been reduced to a point, and Jezebel is no longer physically modeled in the geometry. 
The second condition is important because the next step to simplify the minimum accident of concern is to use the neutron and photon leakage spectra of the critical assembly as the energy dependent portion of a simplified point source. In Sections 4 and 5, the spectra of neutrons and photons used in the SCALE fixed-source calculations are the spectra of neutrons and photons born from fission. In the Jezebel example, more of the fissions occur near the center of the plutonium sphere than near the outer surface of the sphere. Therefore, particles exiting Jezebel most likely have scattered and no longer have the same energy distribution as fission neutrons and photons. Since the physical model of the critical assembly is reduced to a point, this requires that the spectra of neutrons and photons emitted by that point match the spectra of neutrons and photons that leak out of the critical assembly. Calculating the leakage spectra of these particles requires one to simulate the interactions of the particles within the critical assembly, which is why this sort of simplification of the minimum accident of concern can be made.

To summarize, the first step to simplify the minimum accident of concern is to reduce the geometry of the critical assembly to a point, which can only be done if the first condition listed above is satisfied. The second step is to use the critical assembly neutron and photon leakage spectra as the energy dependent portion of the simplified point source. The second step can only be accomplished easily if the second condition listed above is satisfied. Section 7 will present an example of calculating the response of a CAAS detector. The methods used in Sections 4 and 5 will be applied to this example in Sections 7.1 and 7.2, and then the simplification discussed in this section will be applied in Sections 7.3 and 7.4.

\section{CALCULATING THE RESPONSE OF A CAAS DETECTOR}

Now that the minimum accident of concern has been established for the credible accident discussed in Section 5 and shown in Figure 3 and Figure 4, the next logical question is whether a CAAS would be able to detect the minimum accident of concern. This question is answered by calculating the response of a CAAS detector due to a critical assembly using the same basic model discussed in Section 5. In Section 3 , the criterion that established the minimum accident of concern, based on ANSI/ANS-8.3-1997 (R2012), is a criticality accident that delivered a dose rate of $0.2 \mathrm{~Gy} / \mathrm{min}$ at two meters from the outer surface of the critical assembly. In Section 5, it has been determined that the minimum accident of concern for Jezebel in the location described in the simple block building is about $8 \times 10^{13}$ fissions per second. In this analysis, a CAAS detector, which consists of a sphere of air with a $30.48 \mathrm{~cm}$ radius, will be placed in one of the upper corners of the block building near the door to the building. This spherical detector, whose size and shape have been chosen arbitrarily, can be seen in Figure 5 (purple sphere).

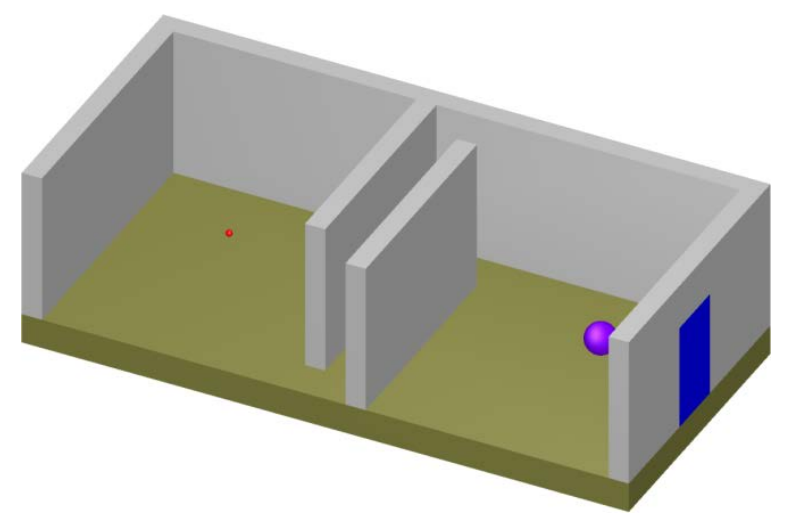

Figure 5. Block building with Jezebel and CAAS detector, roof and front wall removed.

The criticality in this analysis is assumed to produce $1 \times 10^{15}$ fissions per second, which is greater than the minimum accident of concern. This fission rate has been arbitrarily selected, but intentionally different 
from the minimum accident of concern, to illustrate that the results of this analysis with $1 \times 10^{15}$ fissions per second can be scaled to different fission rates. Scaling will allow one to determine if the minimum accident of concern is detectable, the smallest detectable fission rate, or the CAAS detector response for any other proposed fission rate. At this point, no assumption has been made about the alarm set point of any CAAS, which is something that can vary between alarm systems and can be changed between different facilities. For the purposes of this analysis and the remainder of this guidance document, the alarm set point for the $30.48 \mathrm{~cm}$ radius spherical air CAAS detector will be $1 \mathrm{mGy} / \mathrm{hr}\left(1.6667 \times 10^{-5}\right.$ $\mathrm{Gy} / \mathrm{min}=0.1 \mathrm{mrad} / \mathrm{hr})$.

\subsection{SCALE 6.1}

In order to calculate the CAAS detector response due to Jezebel undergoing $1 \times 10^{15}$ fissions per second, the SCALE CAAS analysis capability will be applied again. The complete SCALE input for this sequence of calculations is included in Appendix A.7.a. The KENO and MT2MSM inputs are identical to those used in Section 5.1. The Monaco input needs to be modified to include the $30.48 \mathrm{~cm}$ radius sphere of air representing a CAAS detector, and the spherical shell (purple in Figure 4) used to tally the dose rate to determine the minimum accident of concern can be removed from the geometry. Like all media used as a region tally in Monaco, the volume of the CAAS detector must be entered on the media card to calculate the flux correctly.

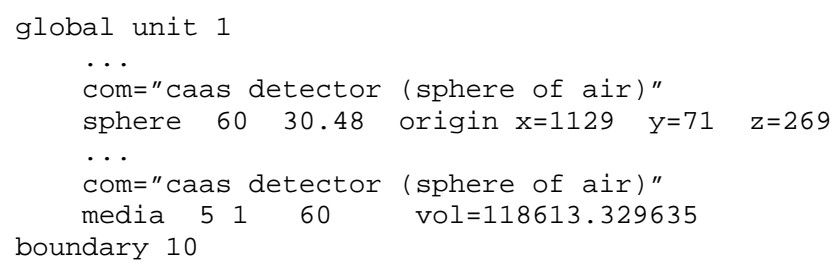

Again, the ICRU air kerma factors available in SCALE need to be requested in the definitions block to serve as the CAAS detector response function. The source will be the same as that used to determine the minimum accident of concern, except the fission rate will be changed to $1 \times 10^{15}$ fissions per second using the "fissions" source keyword.

The region tally of the Monaco model discussed in Section 5.1 must be modified to tally air kerma in the spherical CAAS detector. Remember, the ICRU air kerma factors are likely not the most appropriate flux-to-dose-rate conversion factors for all CAAS detectors. The correct region number to use is 10 (the CAAS detector sphere is defined by the $10^{\text {th }}$ media card in unit 1 ). As before, the "noFissions" keyword must be used in the parameters block because this system is critical, and the "noSecondaries" keyword is not used because photon air kerma rates are being calculated.

Several comments have been made in previous sections (4, 4.2, and 6) about the ability to more easily apply variance reduction techniques to fixed-source calculations than to eigenvalue calculations when calculating a CAAS detector response. Therefore, the SCALE input file in Appendix A.7.a was modified to apply the FW-CADIS automated variance reduction technique (brief description and references in Section 4.1) available in MAVRIC. This new input file is available in Appendix A.7.b. FW-CADIS is applied because this problem contains a single region tally with two flux-to-dose-rate conversion factors (neutron and photon air kerma). The remainder of this section discusses the differences between the MAVRIC input in Appendices A.7.a and A.7.b.

The KENO and MT2MSM input do not need to be modified to apply the FW-CADIS variance reduction technique in MAVRIC. The first change needed is at the top of the MAVRIC/Monaco portion of the input file. Up to this point, the MAVRIC/Monaco calculations have used the 200-neutron/47-photon group cross-section library, which is based on ENDF/B-VII.0 (v7-200n47g). The library designation at 
the top of the MAVRIC input is now changed to the 27-neutron/19-photon group cross-section library, also based on ENDF/B-VII.0 (v7-27n19g). This change is made because the library designation at the top of the MAVRIC input specifies which library is used by the deterministic code Denovo in the MAVRIC sequence to calculate the problem importance map (adjoint fluxes). A separate cross-section library can be designated for the Monaco portion of the MAVRIC sequence later in the input. If this additional library designation is not provided, then the cross-section library designation at the top of the MAVRIC input is also used for the Monaco portion of the calculation. The coarse group library is typically used with Denovo simply to speed up the deterministic calculation. Since the deterministic solution is only being used to generate an importance map, which is used only to accelerate the forward Monte Carlo calculation, an approximate solution is adequate. The material composition and geometry descriptions in MAVRIC do not need to be modified. However, the MAVRIC definitions block needs to be modified. A location is added which represents the center of the spherical CAAS detector. This location will be used later in the input to designate the location of the adjoint point sources for the Denovo calculation. A grid geometry is also added, which will be designated as the Cartesian mesh for the Denovo deterministic calculations later in the input. Good grid geometries will capture significant material boundaries, include mesh lines at the boundary of volumetric forward sources and region tallies, and include more mesh lines where the adjoint flux is changing quickly over short distances. The grid geometry input shown below is illustrated in Figure 6. The view of Jezebel is obscured by the mesh lines because the mesh used in KENO to collect the fission source data is a subset of the mesh Denovo uses, which prevents any loss of fission source data calculated by KENO.

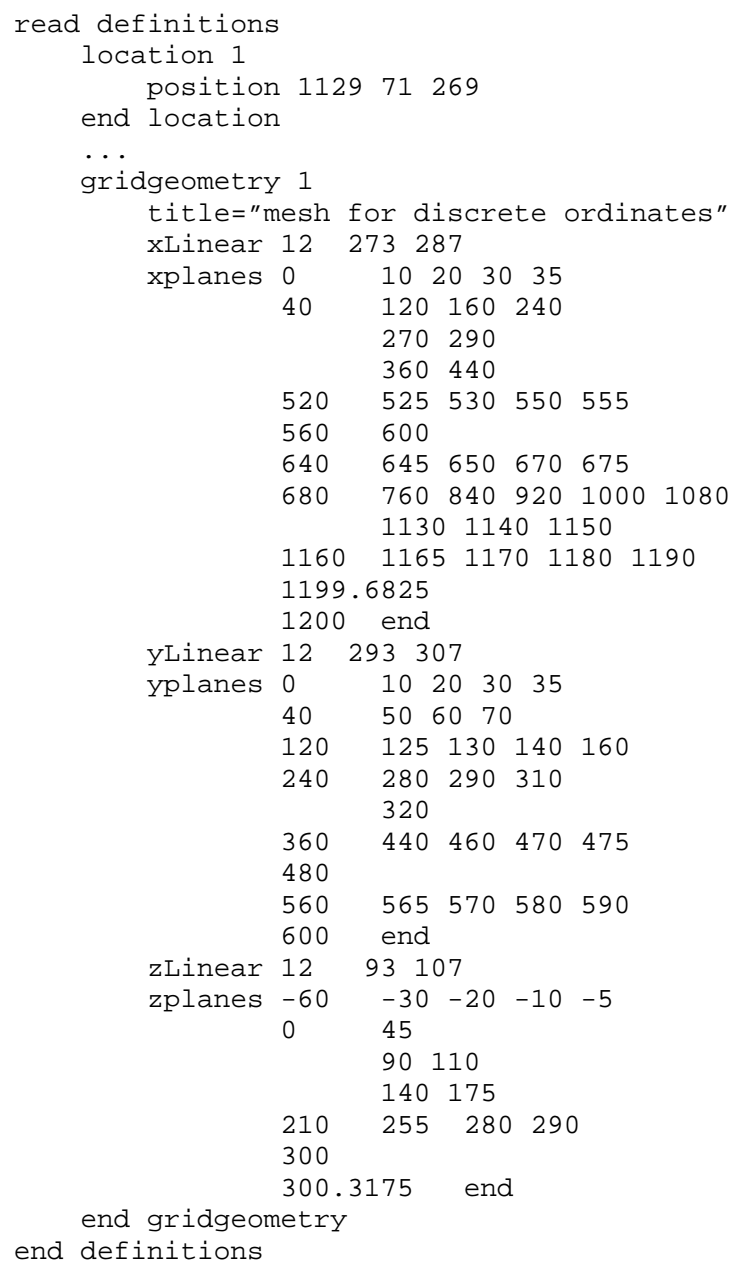




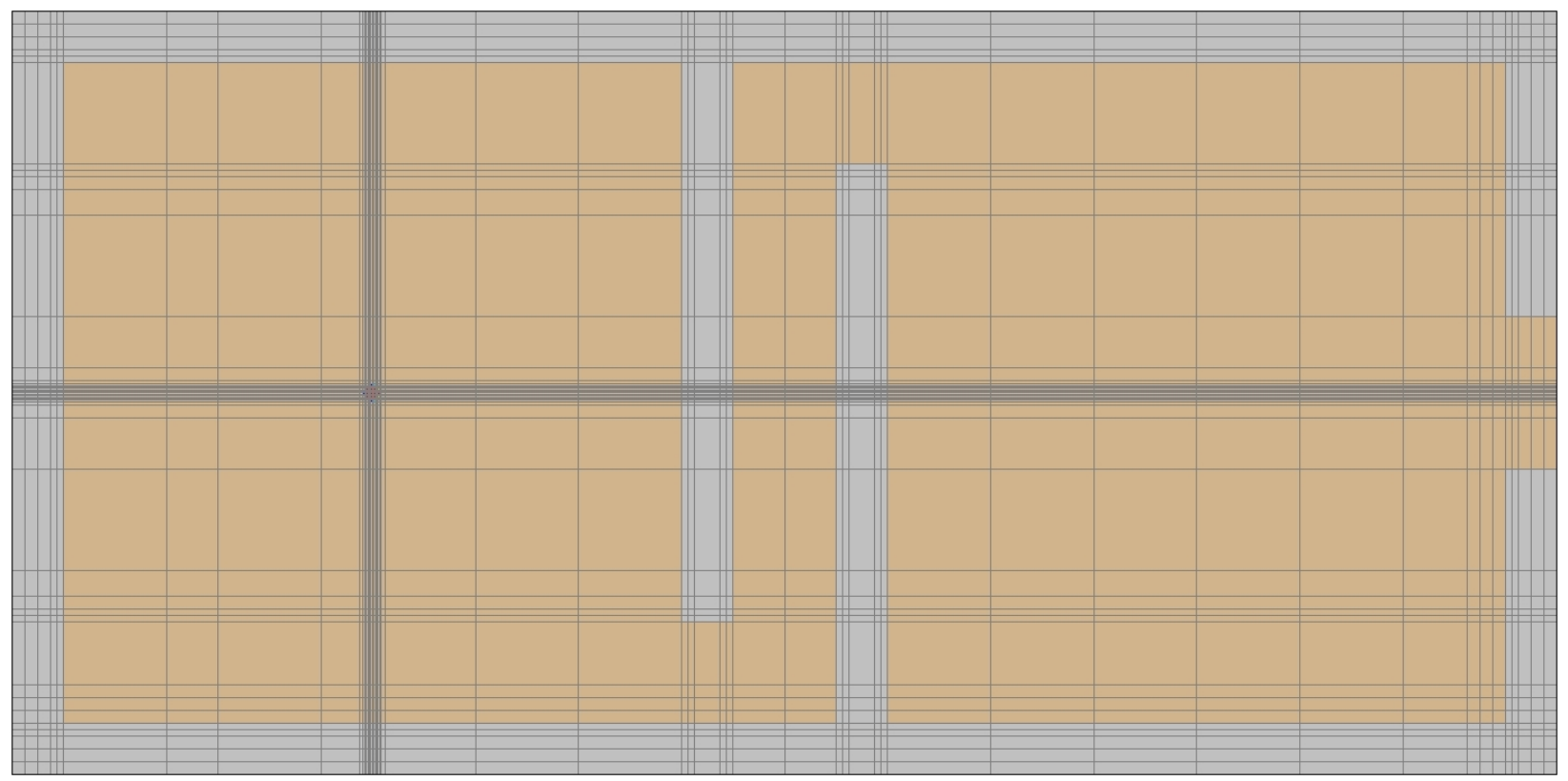

Figure 6. Grid geometry employed by Denovo deterministic calculations.

The source and tally description are completely unchanged when applying FW-CADIS. The parameters block contains only a single change.

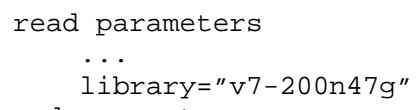

The library keyword is set to v7-200n47g to specify the fine group library to be used with the Monaco calculation during the execution of the MAVRIC sequence. This keyword must be added because the cross section library designation at the top of the input was changed to a coarse group library. If this keyword in the parameters block is omitted, the library designated at the beginning of the input is used for both the deterministic Denovo calculation and the Monaco Monte Carlo calculation. As stated previously, the coarse group library is preferred with Denovo to reduce the run time, but the fine group library is preferred with Monaco to improve the accuracy of the calculation. The final modification is to replace the biasing block (read biasing...) with the importanceMap block.

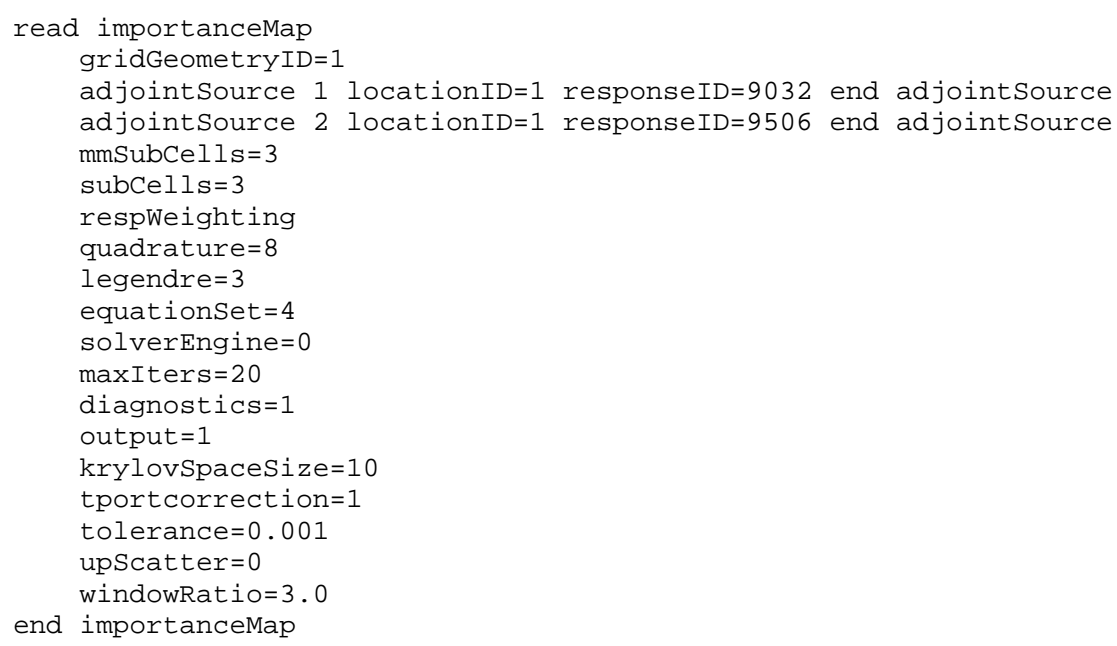


Nearly all of the inputs in the above importanceMap block are perfectly acceptable default values, which are all discussed in the MAVRIC portion of the SCALE manual. However, a few important input parameters from the importanceMap block warrant more discussion. First, the grid geometry that Denovo will use is set (gridGeometryID=1). Two adjoint sources, one for each tally flux-to-dose-rate conversion factors, are placed as point sources at the center of the CAAS detector sphere using location 1, which is specified in the definitions block. If additional or different CAAS detectors are modeled in the tallies block, then the adjoint sources in the importanceMap block must be changed accordingly in order to apply any variance reduction technique to those tallies. Finally, the keyword "respWeighting" tells MAVRIC to apply the FW-CADIS variance reduction technique by optimizing the total response (response integrated over energy) in the Monaco Monte Carlo calculation instead of the energy dependent response or the flux.

\subsection{MCNP5}

Similar to the SCALE calculation of the CAAS detector response to Jezebel undergoing $1 \times 10^{15}$ fissions per second, the MCNP model of the Jezebel benchmark needs to be modified to include the $30.48 \mathrm{~cm}$ sphere of air that represents the CAAS detector. The spheres used to calculate the minimum accident of concern may be deleted. The complete input file that shows all of these geometry changes is available in Appendix A.8. The volume card included in Section 5.2 has also been removed because MCNP can calculate the volume of the spherical CAAS detector tally cell. The only other change required of the MCNP input used in Section 5.2 is to the tally multiplier card (FM). In the previous calculation, the multiplier used represented 1.0 fission per second. This card now needs to reflect the fact that the assumed fission rate is $1 \times 10^{15}$ fissions per second.

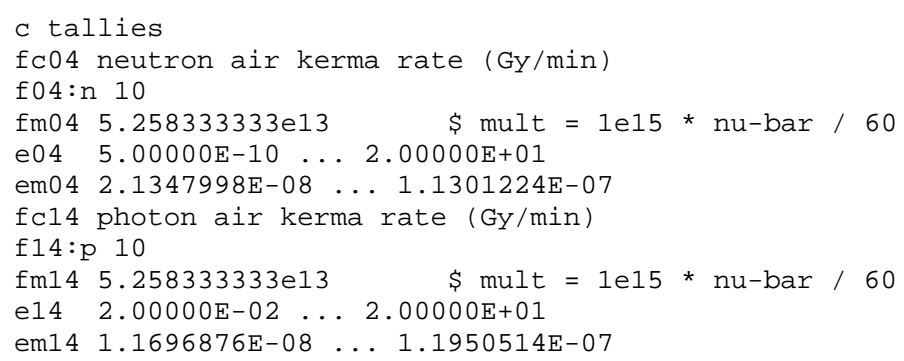

\subsection{SCALE 6.1 - CRITICALITY TREATED AS A POINT}

Up to this point in Section 7, the Jezebel critical assembly has been modeled explicitly when calculating the CAAS detector response. The SCALE calculation described in Section 7.1 applied the CAAS analysis capability, which used KENO to generate a spatial- and energy-dependent fission source that MAVRIC/Monaco used to calculate the CAAS detector response (with and without automated variance reduction). The MCNP calculation described in Section 7.2 is an eigenvalue calculation that also tallied the CAAS detector response, but this calculation did not apply any automated variance reduction. This section and the next describe SCALE and MCNP calculations that simplify Jezebel and treat it as a point source to calculate the CAAS detector response. This is not particularly interesting for SCALE calculations with a single CAAS detector in a fixed location because the SCALE CAAS analysis capability already provides an automated method to generate a fully spatial- and energy-dependent fission source that can be used in a fixed-source calculation. However, this is a useful simplification if a practitioner wants to use a fixed-source MCNP calculation to calculate the CAAS detector response. This method will first be applied with SCALE, even though it is not necessary since the position of the only CAAS detector is already known, to show that the method can be applied with SCALE, MCNP, or any other radiation transport code. 
The two conditions discussed in Section 6 necessary to simplify the critical assembly to a point source have been met. The distance between Jezebel and the CAAS detector is much larger than 7 to 10 times the diameter of Jezebel, the volume of Jezebel is very small compared to the room in which it is located, and Jezebel is not located near a dense reflector. A detailed analysis of these conditions is presented in Appendix B.1, which is a useful example of how to evaluate these conditions. Having satisfied these conditions, the spectra of neutrons and photons leaking from Jezebel need to be calculated. With SCALE, this step still requires the CAAS analysis capability, which is why this approximation is not particularly useful when using SCALE to perform CAAS analysis with a single detector in a fixed location. Furthermore, Monaco only has tallies that calculate the flux within a volume, at a point, or within a Cartesian or cylindrical mesh, so calculating the leakage (or current) with Monaco is not impossible but is somewhat awkward. The detailed discussion of the method and Monaco input file used to calculate the neutron and photon leakage from Jezebel is presented in Appendix B.2. The remainder of this section will simply use the results of the calculations discussed in Appendix B.2.

The numbers of neutrons and photons that leaked from Jezebel as calculated by SCALE using Monaco are given below in Table 3. The spectra are plotted along with the MCNP results in Figure 7 and Figure 8. The MCNP model is discussed in the next section, and the differences between the plots in Figure 7 and Figure 8 are discussed in the summary (Section 7.5).

Table 3. Total leakage from Jezebel as calculated by SCALE (relative errors are at the 1- $\sigma$ level).

\begin{tabular}{|l|l|}
\hline Neutrons per fission per second & $2.12641 \mathrm{E}+00 \pm 0.007 \%$ \\
\hline Photons per fission per second & $5.87772 \mathrm{E}-01 \pm 0.04 \%$ \\
\hline
\end{tabular}

The leakage results (number and spectra of neutrons and photons) are used as source input in a model nearly identical to the MAVRIC/Monaco model from Section 7.1. The primary difference is that the source is now described as a point. The full input file for this calculation is in Appendix A.9.b, which is a modification of the MAVRIC/Monaco input in Appendix A.7.b discussed in Section 7.1 that applied the FW-CADIS method. The 27-neutron/19-photon group library will be used for the Denovo calculations, but the 200-neutron/47-photon group library will be used for the Monaco calculations. The geometry and materials are identical to those used in Section 7.1, but the plutonium composition and physical description of Jezebel has been deleted from the model. In the Monaco definitions block, two new distributions are needed to specify the energy of the neutrons and photons in the source. These distributions are exactly the leakage spectra calculated in Appendix B.2. Below is an abbreviated version of these lines of input.

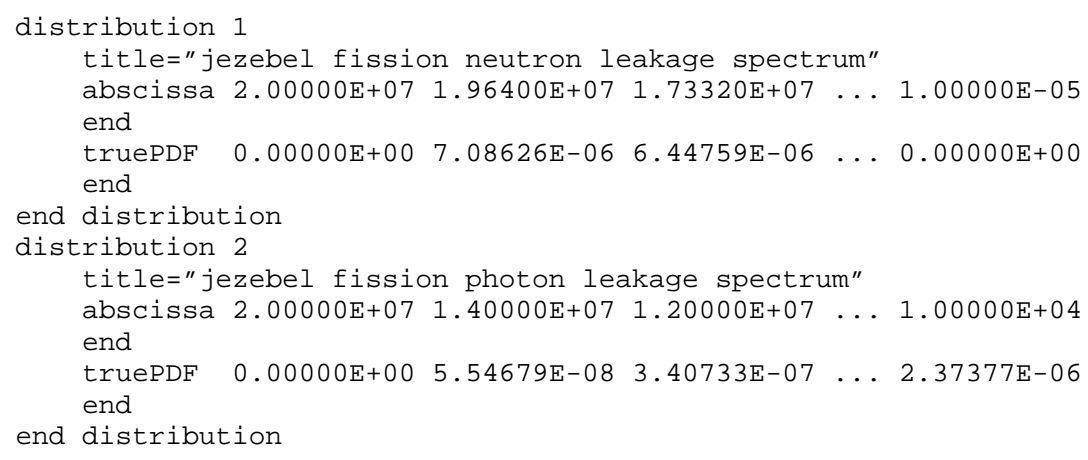

Then the description of the source is modified to describe two point sources located at the center of where Jezebel was formerly modeled, and these sources (one neutron and one photon) require the new distributions entered in the definitions block. 


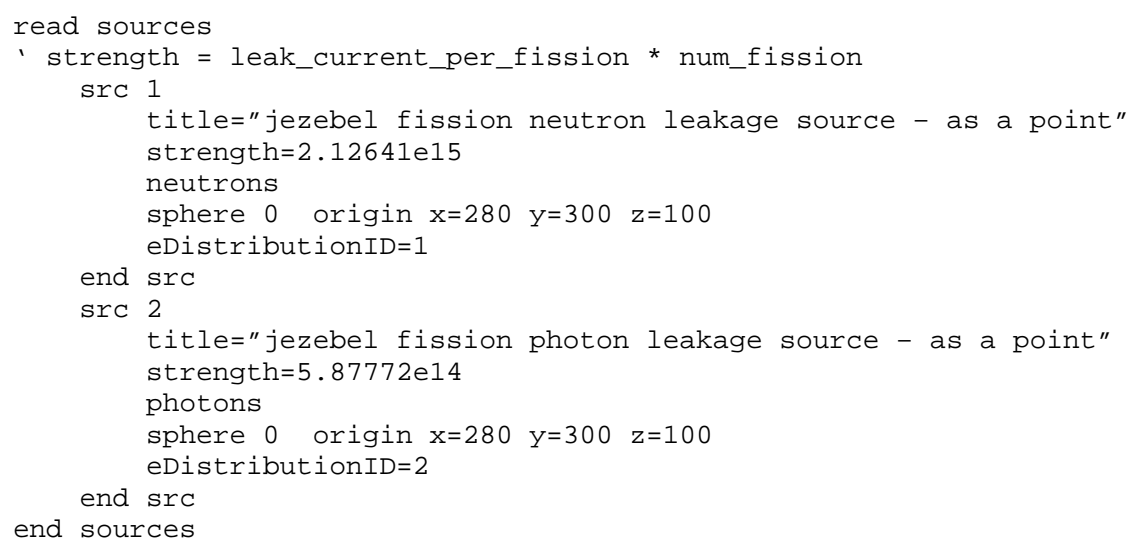

Notice that the strength of each source is equal to the total number of leakage particles reported in Table 3 multiplied by the number of fissions, which for this analysis was assumed to be $1 \times 10^{15}$ fissions per second. The remainder of this input file (the tallies, the parameters block, and the importance map block) remains unchanged from the input in Appendix A.7.b.

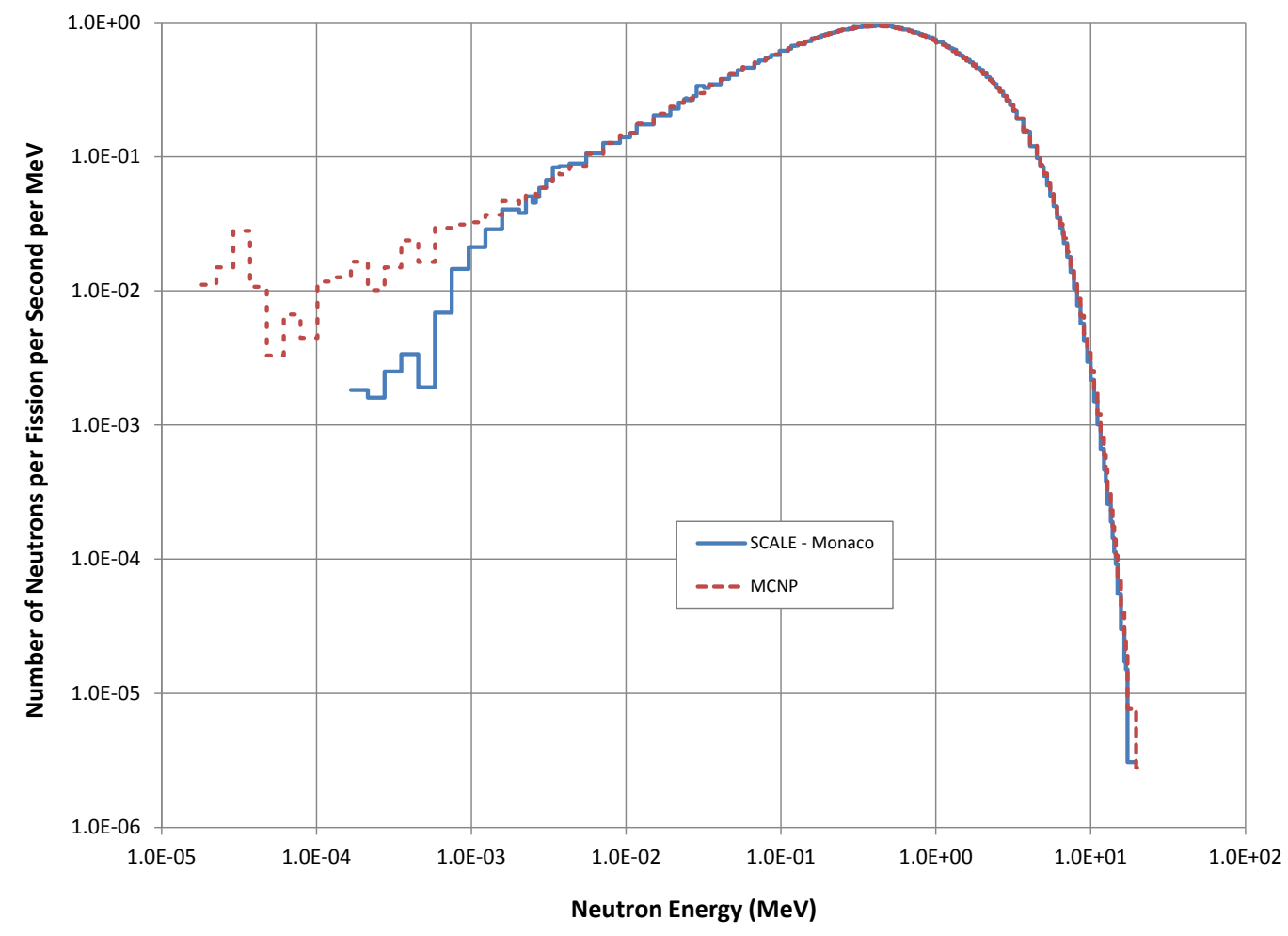

Figure 7. Comparison of SCALE and MCNP neutron leakage spectra. 


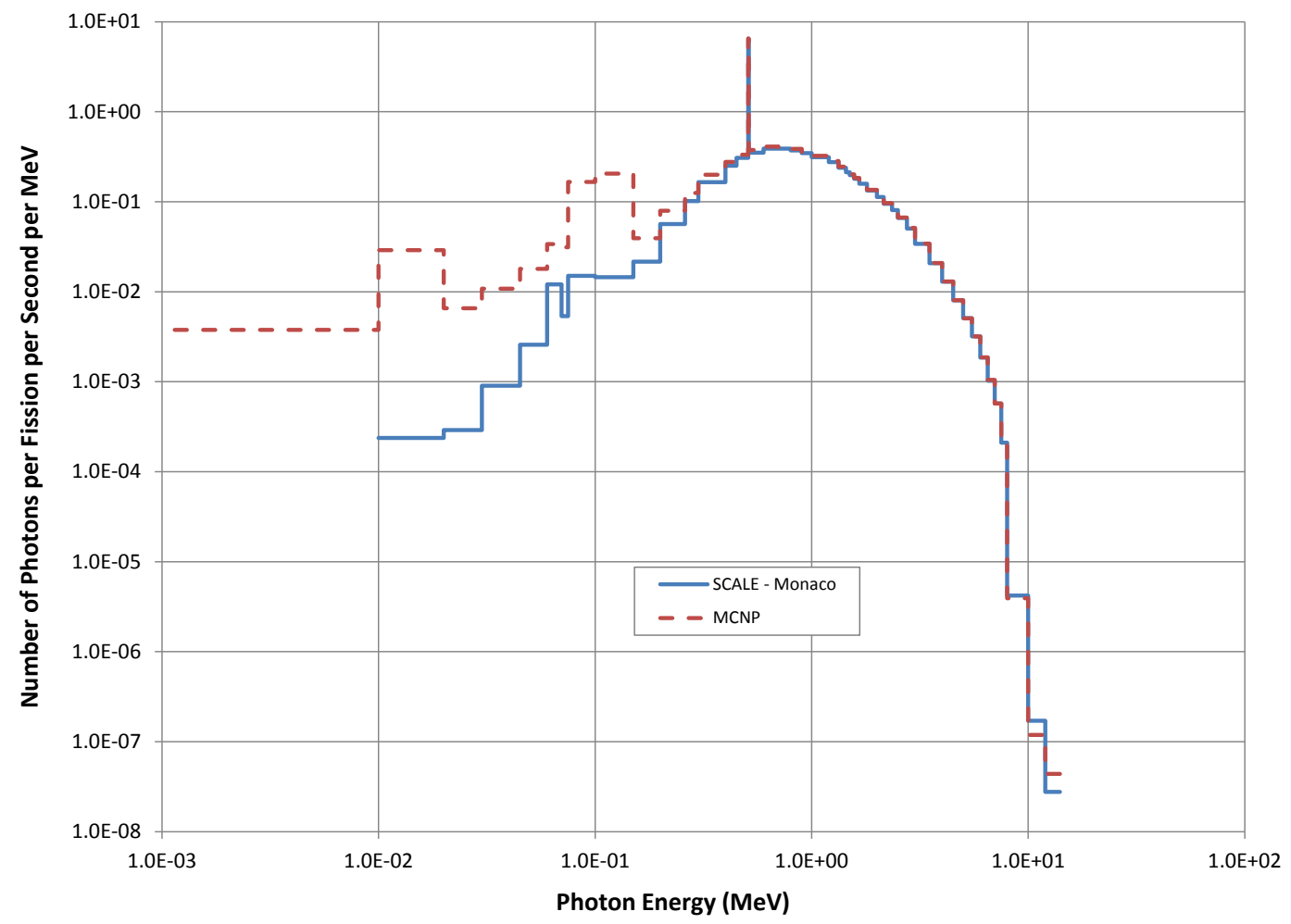

Figure 8. Comparison of SCALE and MCNP photon leakage spectra.

\subsection{MCNP5 - CRITICALITY TREATED AS A POINT}

First, the number and spectra of neutrons and photons leaking from Jezebel need to be calculated with MCNP. MCNP does have a current tally (F1 tally); therefore, the efforts made in the Monaco model to make a volumetric flux tally behave like a current tally are not necessary. The process by which this is accomplished with MCNP will not be discussed in a separate appendix because the requisite discussion is brief. The neutron and photon leakage spectra will simply be tallied on the surface of Jezebel. The MCNP input file used in Section 7.2 is a good starting point. Similar to the SCALE analysis, the MCNP geometry should only include Jezebel, and all materials other than the plutonium mixture can be deleted. The full input file for this analysis is included in Appendix A.10.a. The lines below show the new MCNP geometry in its entirety.

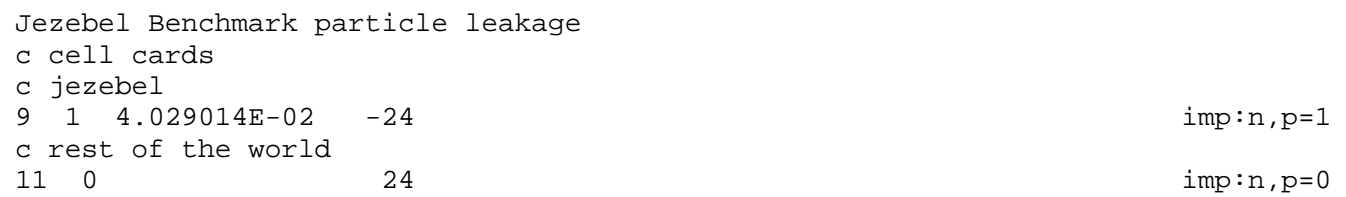

F1 tallies of the current on the surface of Jezebel replace the F4 tallies used previously in Section 7.2. The same energy bins (E card) are used, but the energy multiplier (EM) cards are not needed because no energy-dependent response is being applied to the calculated current. The value of the tally multiplier (FM card) has been set to the MCNP value of $\bar{v}$. The current is calculated on a per fission basis, eliminating the need for the $1 \times 10^{15}$ multiplier applied to the Section $7.2 \mathrm{FM}$ card, and the division by 60 is unnecessary because the flux-to-dose-rate conversion factors have been removed. 
c tally cards

fc1 jezebel neutron leakage (neutrons/sec per fission/sec)

f1: $\mathrm{n} 24$

fm1 3.155

e1 $1.00000 \mathrm{E}-11 \ldots 2.00000 \mathrm{E}+01$

fc11 jezebel photon leakage (photons/sec per fission/sec)

f11: $p 24$

fm11 3.155

e11 1.00000E-02 K. 2.00000E+01

The numbers of neutrons and photons that leaked from Jezebel as calculated by MCNP are given in Table 4. The spectra are plotted, along with the SCALE results, in Figure 7 and Figure 8.

Table 4. Total leakage from Jezebel as calculated by MCNP

(relative errors are at the $1-\sigma$ level).

\begin{tabular}{|l|l|}
\hline Neutrons per fission per second & $2.12373 \mathrm{E}+00 \pm<0.01 \%$ \\
\hline Photons per fission per second & $6.27838 \mathrm{E}-01 \pm 0.02 \%$ \\
\hline
\end{tabular}

To calculate the CAAS detector response with Jezebel modeled as a point source, the number and spectra of neutrons and photons calculated with the bare Jezebel model are used as sources in two models nearly identical to the MCNP model from Section 7.2. Two models must be used because MCNP5 only allows for one source particle type using the general source (SDEF). This is not the case for MCNPX, but this analysis uses MCNP5. First, the neutron leakage is modeled as a point source (input available in Appendix A.10.b.1), and then the photon leakage is modeled (input available in Appendix A.10.b.2). These input files are modifications of the MCNP input in Appendix A.8, which was discussed in Section 7.2. The geometry and materials are identical to those used in Section 7.2, but the plutonium composition and physical description of Jezebel have been deleted from the model. The major difference between these two MCNP calculations and all of the MCNP calculations discussed previously in this guidance document is that these are fixed-source calculations. Therefore, the KCODE and KSRC cards are deleted from the input, and are replaced by the general source SDEF card. The source is a point source (neutron in one input and photon in the other) located where the center of Jezebel was located in previous models. The source particle energy is sampled from a distribution entered using the SI and SP cards, where the data values entered are exactly the leakage spectra calculated previously in this section. An abbreviated version of the neutron source is given next.

c source cards

sdef erg $=$ d1 pos $=280300100$ par $=\mathrm{n}$

Si1 H 1.00000E-25 ... 1.73320E+01 1.96400E+01 2.00000E+01

sp1 D $\odot \quad 0.00000 \mathrm{E}+00 \ldots 8.29292 \mathrm{E}-06$ 1.76654E-05 1.00265E-06

Below is an abbreviated version of the photon source.

c source cards

sdef erg=d1 pos $=280300100$ par $=p$

si1 H 1.00000E-25 ... 1.20000E+01 1.40000E+01 2.00000E+01

Sp1 D $\odot ~ 3.38493 E-05 \quad \ldots \quad 2.38039 E-07 \quad 8.79607 E-08 \quad 0.00000 E+00$

The tallies in these two MCNP calculations are essentially the same as those used in Section 7.2, with the exception of the tally multipliers. The calculation with the neutron source needs a neutron and photon dose rate tally. However, the calculation with the photon source only needs a photon tally. Photonuclear physics is ignored because photoneutron production compared to fission is negligible for these types of problems. The flux-to-dose-rate conversion factors used for these tallies are identical to those in Section 7.2 (same E and EM cards). However, the tally multiplier (FM card) must be updated and will be different between the calculations with the neutron and photon source. An abbreviated view of the tallies in the neutron source calculation is shown below. 


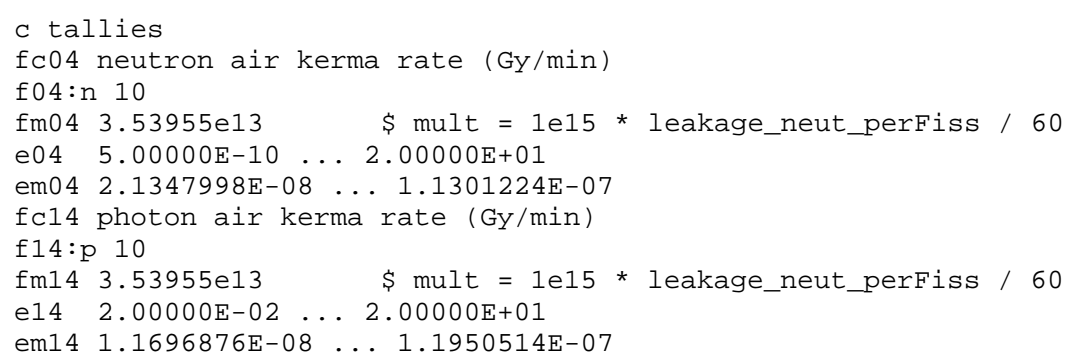

Next is an abbreviated view of the tally in the photon source calculation.

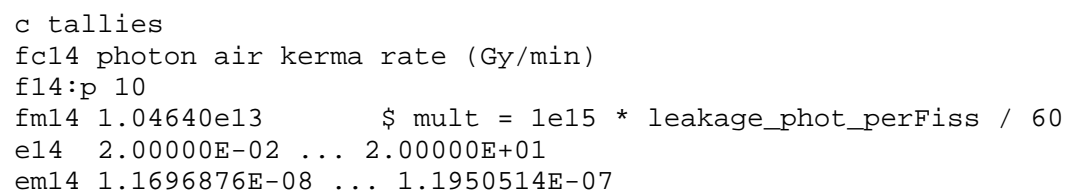

Notice that the tally multiplier is equal to the total number of leakage particles reported in Table 4 times the number of fissions, which for this analysis was assumed to be $1 \times 10^{15}$ fissions per second, divided by 60 to calculate the dose rate with units of per minute. The leakage value used from Table 4 on the tally multiplier card corresponds to the source particle type, regardless of the type of particles being tallied. If MCNPX were used such that both particle types (neutron and photon) were modeled by the source, then the tally multiplier would use the sum of the neutron and photon leakage in Table 4 . The remainder of this input file remains unchanged from the input in Appendix A.8.

\subsection{SUMMARY}

Section 7 describes a simple example of how to calculate a CAAS detector response due to a critical assembly using SCALE and MCNP. This is accomplished by using a fully energy- and spatial-dependent fission source, and by simplifying the fission source to an energy-dependent point source that models the leakage from the critical assembly. Some intermediate results have already been presented in Table 3 and Table 4 and Figure 7 and Figure 8. Comparing the neutron leakage calculated by SCALE (Table 3) and MCNP (Table 4), the SCALE value is larger by less than $0.2 \%$. Figure 7 shows that the spectrum of neutrons leaking from Jezebel calculated by SCALE and MCNP is very similar (the differences at low energies, around $1 \mathrm{keV}$, are due to statistical uncertainty). The photon leakage calculated by MCNP is larger than the SCALE value by about 7\%. Considering the differences in photon physics between MCNP and SCALE, this difference in photon leakage is understandable. Figure 8 illustrates the photon spectral differences due to the difference between MCNP and SCALE photon physics. In Figure 8, one can see that SCALE and MCNP correctly model the photon peak due to positron annihilation. The differences in Figure 8 are due to the MCNP thick-target bremsstrahlung model that produces low energy photons not modeled by SCALE and the SCALE $10 \mathrm{keV}$ photon cutoff (compared to $1 \mathrm{keV}$ in MCNP).

All of the methods applied in Section 7 provided very similar results, which is evident by the results in Table 5. The SCALE neutron and photon CAAS detector responses in Table 5 are all statistically the same, regardless of how the source was modeled or what variance reduction techniques were applied. Likewise, the MCNP neutron and photon CAAS detector responses in Table 5 are all statistically the same, regardless of how the source was modeled or what variance reduction techniques were applied. On the $95 \%$ confidence interval $(2-\sigma)$ the SCALE and MCNP neutron CAAS detector responses are also statistically equivalent. The MCNP photon CAAS detector responses are expected to be larger than the SCALE photon CAAS detector responses, which they are. The largest MCNP photon CAAS detector response is nearly 31\% greater than the smallest SCALE photon CAAS detector response. This difference between the SCALE and MCNP photon results is consistent with the difference in the photon dose rate per fission and the minimum accident of concern between SCALE and MCNP discussed in 
Section 5.3. Also displayed in Table 5 is the figure-of-merit (FOM), which is a measure of the efficiency of the Monte Carlo calculation. High FOMs represent more efficient Monte Carlo simulations [2,3].

Table 5. Comparison of calculated CAAS detector responses using different radiation transport codes, critical source approximations, and variance reduction (relative errors are at the 1- $\sigma$ level).

\begin{tabular}{|c|c|c|c|c|c|}
\hline Result & Source Model & $\begin{array}{l}\text { Variance } \\
\text { Reduction }\end{array}$ & $\begin{array}{l}\text { CAAS Response } \\
\text { (Gy/min) }\end{array}$ & $\begin{array}{l}\text { CPU Time } \\
(\mathrm{min})\end{array}$ & $\begin{array}{l}\text { FOM } \\
(/ \mathrm{min})\end{array}$ \\
\hline SCALE Neutron & Detailed & Semi-analog & $3.6502 \mathrm{E}-02 \pm 0.81 \%$ & 745 & 20.2 \\
\hline SCALE Photon & Detailed & Semi-analog & $1.6735 \mathrm{E}-02 \pm 1.81 \%$ & 745 & 4.08 \\
\hline SCALE Neutron & Detailed & FW-CADIS & $3.6663 \mathrm{E}-02 \pm 0.46 \%$ & $175^{\mathrm{a}}$ & $268^{a}$ \\
\hline SCALE Photon & Detailed & FW-CADIS & $1.6550 \mathrm{E}-02 \pm 2.04 \%$ & $175^{\mathrm{a}}$ & $13.8^{\mathrm{a}}$ \\
\hline SCALE Neutron & Leakage/Point & FW-CADIS & $3.6847 \mathrm{E}-02 \pm 0.47 \%$ & $177^{\mathrm{a}}$ & $252^{\mathrm{a}}$ \\
\hline SCALE Photon & Leakage/Point & FW-CADIS & $1.6957 \mathrm{E}-02 \pm 1.69 \%$ & $177^{\mathrm{a}}$ & $19.8^{\mathrm{a}}$ \\
\hline MCNP Neutron & Detailed & Semi-analog & $3.7147 \mathrm{E}-02 \pm 0.76 \%$ & 703 & 24.6 \\
\hline MCNP Photon & Detailed & Semi-analog & $2.1512 \mathrm{E}-02 \pm 1.72 \%$ & 703 & 4.81 \\
\hline MCNP Neutron & Leakage/Point & Semi-analog & $3.7340 \mathrm{E}-02 \pm 0.68 \%$ & 896 & 24.1 \\
\hline MCNP Photon & Leakage/Point & Semi-analog & $2.1662 \mathrm{E}-02 \pm 1.32 \%$ & $995^{b}$ & $5.81^{b}$ \\
\hline MCNP Neutron & Leakage/Point & FW-CADIS & $3.6811 \mathrm{E}-02 \pm 0.44 \%$ & $258^{\mathrm{a}}$ & $200^{\mathrm{a}}$ \\
\hline MCNP Photon & Leakage/Point & FW-CADIS & $2.1418 \mathrm{E}-02 \pm 1.16 \%$ & $266^{\mathrm{a}, \mathrm{b}}$ & $28.1^{\mathrm{a}, \mathrm{b}}$ \\
\hline
\end{tabular}

bValues include the MCNP run time for both the neutron and photon source calculations

The real purpose of the calculations in this section is to answer the question, "Will the CAAS, in the right room of Figure 5, alarm if Jezebel, in the left room of Figure 5, is undergoing $1 \times 10^{15}$ fissions per second and the CAAS alarm set point is $1 \mathrm{mGy} / \mathrm{hr}\left(1.6667 \times 10^{-5} \mathrm{~Gy} / \mathrm{min}=0.1 \mathrm{mrad} / \mathrm{hr}\right)$ ?” The results in Table 5 show that the answer to that question is most definitely yes.

The next logical question is, "Will this CAAS alarm for the minimum accident of concern?" This question can be answered by scaling the results in Table 5 via Eq. (2),

$$
R_{M A O C}=\frac{R_{1 e 15}}{1 \times 10^{15}} * M A O C,
$$

where $R_{1 e 15}$ is one of the CAAS detector responses from Table 5 and $R_{M A O C}$ is the CAAS detector response due to the minimum accident of concern. The CAAS detector responses in Table 5 are all based on Jezebel undergoing $1 \times 10^{15}$ fissions per second. All of the CAAS detector responses in Table 5 can be scaled to either of the minimum accidents of concern in Table 2 and the resulting response will be greater than $1.6667 \times 10^{-5} \mathrm{~Gy} / \mathrm{min}$. Therefore, the CAAS detector in Figure 5 will definitely alarm for the minimum accident of concern.

The final question that is likely to be asked is, "What is the smallest accident (fission rate) that can be detected by the configuration shown in Figure 5?" This question can be answered by scaling the data reported in Table 5 to the CAAS alarm set point via Eq. (3),

$$
S D A=\frac{1 \times 10^{15}}{R_{1 e 15}} * R_{\text {alarm }}
$$

where $S D A$ is the smallest detectable accident and $R_{\text {alarm }}$ is the CAAS alarm set point. Table 6 provides the smallest detectable accidents for Jezebel in Figure 5, assuming the CAAS detector responds to neutrons, photons, or both. Simulation results in Table 5 that modeled the source with full detail and did not apply FW-CADIS, i.e. semi-analog, have been used to calculate the fission rates in Table 6 . 
Table 6. Smallest detectable fission rate for the Figure 5 credible accident configuration

\begin{tabular}{|l|l|l|}
\hline Detected Particle Type & SCALE (fissions/sec) & MCNP (fissions/sec) \\
\hline Neutron & $4.5661 \mathrm{e} 11$ & $4.4868 \mathrm{e} 11$ \\
\hline Photon & $9.9594 \mathrm{e} 11$ & $7.7478 \mathrm{e} 11$ \\
\hline Neutron \& Photon & $3.1307 \mathrm{e} 11$ & $2.8413 \mathrm{e} 11$ \\
\hline
\end{tabular}

As expected, the smallest detectable accident calculated by SCALE and MCNP is very similar if the CAAS response is based on detecting neutrons. The SCALE value is nearly $2 \%$ larger than the MCNP value. Since MCNP models more photon physics than SCALE, MCNP calculated a larger photon dose deposited per fission. Therefore, if the CAAS response is based on detecting photons, the smallest detectable fission rate calculated by MCNP is smaller than that calculated by SCALE. The SCALE value is about 29\% larger than the MCNP value. If the CAAS detector were able to respond to both neutrons and photons, the smallest detectable fission rate calculated by SCALE is about $10 \%$ greater than the MCNP value. Regardless of what code is used or what particle type is detected, the smallest detectable fission rate is much less than the minimum accident of concern reported in Table 2, both of which are approximately $8 \times 10^{13}$ fissions per second.

\section{EVALUATING THE COVERAGE OF A CAAS DETECTOR}

The simulations performed so far have calculated the dose response in a specific detector volume due to a credible accident with a specific critical assembly in a fixed location, so these calculations have simulated a very specific source and detector configuration. The specificity of this configuration can be relaxed by applying a mesh tally rather than a single tally representing the response of a CAAS at one location. The previous simulations, particularly in Section 7, model the CAAS detector as a sphere of air, and a volume or region tally was used to calculate the detector response. A mesh tally is simply an array of region tallies, so a mesh tally could be used to tally the dose rate everywhere inside the block building in Figure 5 , instead of using a region tally that covers only the detector sphere. This would allow the practitioner to relax the specific location and response of the CAAS detector. With the mesh tally results, the practitioner would be able to state the detector response anywhere inside the block building due to a specified critical assembly and location. This information would allow the practitioner to determine the detector locations that would and would not provide coverage of (detect) the specific credible accident. This type of analysis is very useful if the credible accident has a well-known critical assembly and fixed location, but the CAAS detector location is unknown. In other words, forward calculations with mesh tallies can be used to answer the question "Where will a CAAS alarm due to a specific credible accident assembly and location?”

To this point, all of the calculations discussed have been forward transport calculations, which model the birth of particles at the critical assembly and transport them throughout the geometry. The flux inside a detector volume (or volumes in the case of a mesh tally) is calculated and convolved with flux-to-doserate conversion factors to calculate the detector response. Another option is to perform adjoint transport calculations, which model the birth of adjoint particles at a detector and transport them throughout the geometry. The adjoint flux inside a detector volume, which corresponds to the critical source assembly, is calculated and convolved with a set of adjoint flux-to-dose-rate conversion factors, which corresponds to the critical source distribution (energy dependent for a point source; energy and spatial dependent for a volumetric source), to calculate the detector response. Similar to using a mesh tally with a forward simulation, a mesh tally with an adjoint simulation allows the practitioner to relax some of the specifics of the source and detector configuration. An adjoint simulation requires the practitioner to describe the location and flux-to-dose-rate conversion factors of a specific CAAS detector, and a mesh tally of the adjoint flux convolved with a critical source provides the practitioner with the detector response anywhere within the mesh tally. This approach allows the practitioner to determine the accident locations that 
would and would not be covered (detected) by a specific detector location. This type of analysis is very useful if the CAAS detector location and response function are well-known, but the credible accident location is unknown. In other words, adjoint calculations can be used to answer the question "Where will a credible accident be detected by a specific CAAS detector type and location?" For adjoint simulations with a mesh tally, the credible accident must be simplified to a point, as described in Section 6. Otherwise, the spatial dependence and physical description of the credible accident must be modeled in every tally location of the adjoint calculation, which would reduce the flexibility provided by this approach.

\subsection{SCALE 6.1 - CAAS COVERAGE EVALUATED BY A FORWARD MESH TALLY}

The problem simulated in Section 7.3 (Jezebel treated as a point with SCALE) is now modified to tally the detector response throughout the block building in Figure 5. The full input file is available in Appendix A.13. All of the geometry cards that refer to the spherical CAAS detector can be deleted, but this is not required. A coarse-grid geometry is already included in the file, which is used by Denovo to calculate adjoint fluxes for the FW-CADIS variance reduction technique. A fine-grid geometry is added to the input file to provide more spatial detail in the dose-rate map created by the mesh tally.

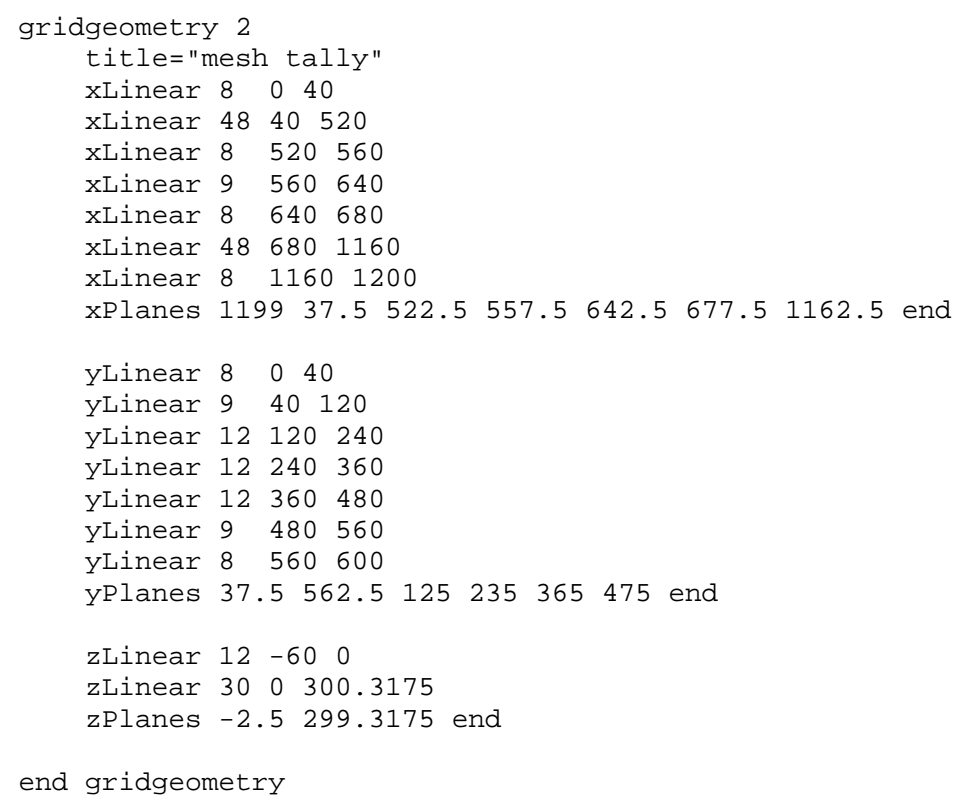

The neutron and photon leakage spectra are unchanged, as are the forward sources. The region tally for the spherical CAAS detector is deleted and a mesh tally covering the entire facility is added. However, note that the dose response calculation is limited to mixture 5, which is the air mixture in this model. This means that dose rates outside of the air (e.g., in the concrete walls) in the model will not be tallied. The fine-grid geometry is specified in the mesh tally definition. The flux-to-dose-rate conversion factors and tally multiplier used in the mesh tally are the same as those used with the region tally in Section 7.3. The noGroupFluxes keyword is used to reduce the size of the mesh tally file. This analysis is only interested in the total neutron and photon CAAS detector response, so detailed results of the neutron and photon flux spectra do not need to be saved. 


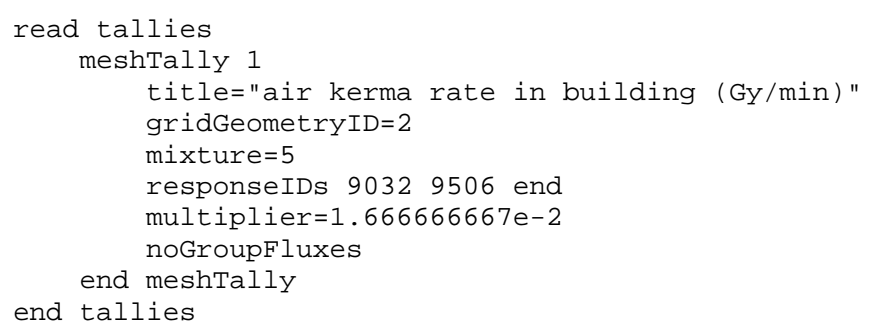

The remainder of the input file, except for the adjoint source description, remains unchanged. The adjoint sources used in Section 7.3 are point sources, which is perfectly acceptable because the tally volume is filled with air and is not very large (when compared to the size of the entire model). If the tally volume in Section 7.3 had been filled with a different material, such as water, or if the volume is much larger such that the flux has a large spatial dependence, a volumetric adjoint source would be more appropriate. For this calculation, the mesh tally covers the entire facility so a volumetric adjoint source is appropriate.

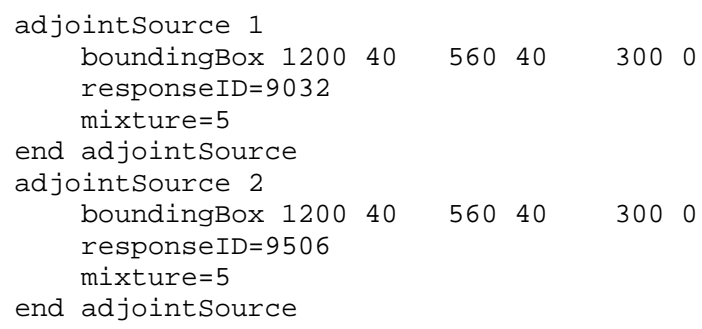

To ensure that these adjoint sources cover the same regions as the mesh tally, a bounding box that matches the inside dimensions of the block building in Figure 5 has been used. The extent of these adjoint sources has also been limited to regions containing mixture 5 to match the mesh tallies, which better optimizes the weight windows and biased source for the problem.

\subsection{MCNP5 - CAAS COVERAGE EVALUATED BY A FORWARD MESH TALLY}

The problems simulated in Section 7.4 (Jezebel treated as a point with MCNP) are modified to tally the detector response throughout the block building in Figure 5. As before, this requires using two MCNP input files because MCNP5 does not allow multiple source particle types. The full input files for this analysis with a mesh tally are in Appendices A.14.a (neutron source) and A.14.b (photon source), which are modifications of the files in Appendices A.10.b.1 and A.10.b.2, respectively. All of the cell and surface cards that refer to the spherical CAAS detector can be deleted, but this is not required. The forward sources (SDEF cards) are unchanged as are the neutron and photon leakage spectra (ERG distributions). The material specifications also remain unchanged.

The only significant required change is to remove the cell tallies for the spherical CAAS detector and replace them with mesh tallies covering the entire facility. The tally multipliers remain unchanged, and the values of the flux-to-dose-rate conversion factors are unchanged. However, mesh tallies in MCNP5 are not compatible with energy multipliers (E and EM cards), so the flux-to-dose-rate conversion factors have to be reformatted as dose energy / dose function input (DE/DF cards). Below is an abbreviated presentation of the neutron and photon mesh tally input for the MCNP input file with the neutron source. 


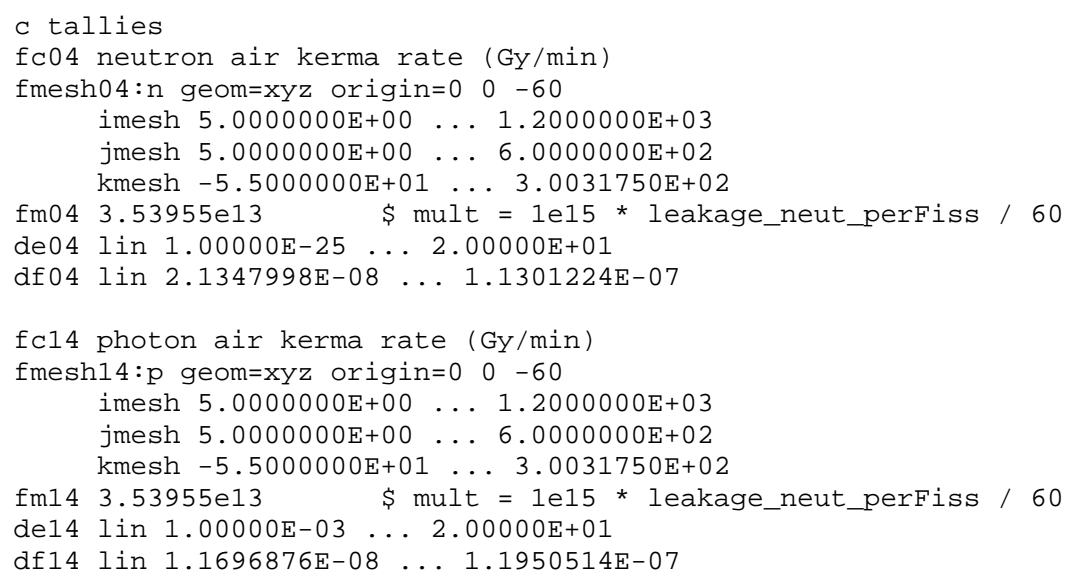

For the MCNP input with the photon source, the neutron tally is not needed (again photonuclear physics is being ignored), and the photon tally is nearly identical to the neutron source case. The photon tally multiplier has to change between these cases because the normalization for the neutron and photon leakage source is different (this is the same as Section 7.4). The abbreviated photon tally for the photon source case is listed below. The tally multiplier (FM card) is the only difference from the neutron source case.

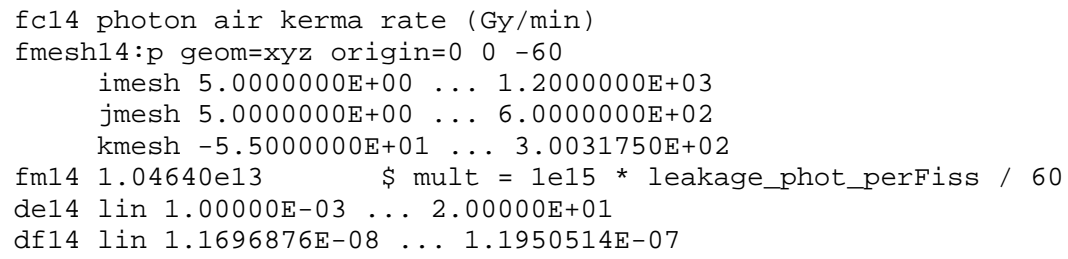

The mesh that describes the spatial domain of these MCNP mesh tallies are provided by the ORIGIN, IMESH, JMESH, and KMESH cards. The mesh lines that have been entered on these cards match the data used in the SCALE mesh tally in Section 8.1. The reason an MCNP5 mesh tally is not compatible with the E and EM cards is in part due to the availability of the EMESH card. The EMESH card allows one to enter an energy mesh that a mesh tally can use to save energy-dependent data. This card has been ignored in this analysis, so only the total neutron and photon CAAS detector response is saved.

\subsection{SCALE 6.1 - CAAS COVERAGE EVALUATED BY AN ADJOINT MESH TALLY}

In SCALE 6.1, the fixed-source Monte Carlo transport code Monaco does not have an adjoint capability. As a proof-of-principal, the adjoint CAAS coverage evaluation will be performed using an approximate Denovo adjoint calculation via the MAVRIC sequence. This Denovo calculation is approximate because detailed deterministic calculations for a facility of the size used in this problem (Figure 5) usually require a large amount of memory and CPU time. The limitations of memory and time can be reduced by the use of parallel computing, but Denovo in SCALE 6.1 is only able to operate on a single CPU. Fully parallel Denovo is available as part of the ADVANTG code.

For this analysis with Denovo, the input discussed in Section 7.3 needs only a few modifications. The full input file for this adjoint Denovo calculation, which is driven by the SCALE MAVRIC sequence, is provided in Appendix A.15. The first two modifications appear at the beginning of the MAVRIC input file. 


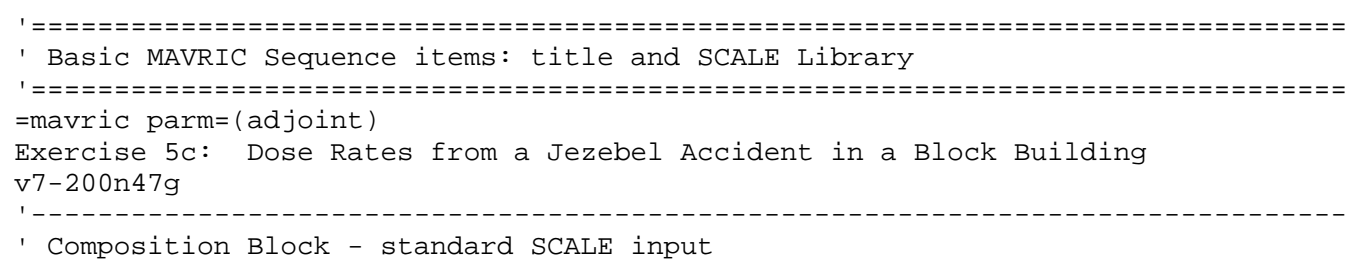

The "parm=adjoint" keyword following the name of the SCALE sequence (=mavric) is a signal to MAVRIC that it should stop after the adjoint Denovo calculation has been performed. The other change is that the fine group shielding library, v7-200n47g, will be used by the Denovo calculation. This library requires more memory than the coarse group library and will make the run time longer, but the Jezebel leakage spectra have been calculated using the fine group structure. Therefore, this group structure will be used to retain the detail of the leakage spectra. The last three modifications are towards the end of the input file in the importanceMap block.

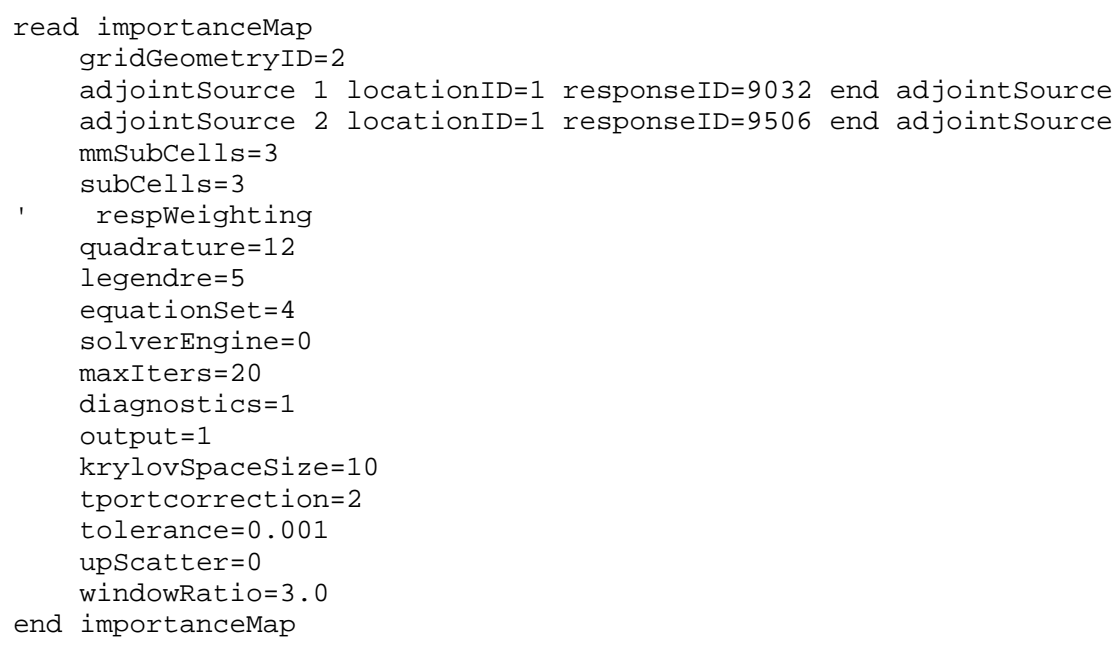

Note that the adjoint Denovo calculation uses the same fine grid geometry as the forward mesh tally in Section 8.1 thereby providing finer detail in the spatial dependence of the adjoint flux. The point adjoint sources, which were replaced by volumetric adjoint sources in Section 8.1, are back because in an adjoint calculation, a specific CAAS detector and location are modeled. In this case, the spatial dependence of the CAAS detector is approximated as a point, which is reasonable for a CAAS detector that is small and consists of air. Finally, the "respWeighting" keyword is commented out because no forward Denovo calculation is needed to weight the adjoint sources. The source strength of the adjoint sources is simply the sum of the group-dependent neutron and photon kerma factors used as the CAAS detector response functions, which will be set by the MAVRIC sequence when building the Denovo input.

\subsection{SUMMARY}

First, note that the MCNP multi-group adjoint transport capability is not compatible with mesh tallies in MCNP5. This discrepancy has been reported to the MCNP developers, and will hopefully be resolved in a future release of MCNP. Therefore, Section 8 does not contain any discussion concerning MCNP and adjoint mesh tallies.

Some of the results of the forward mesh tally calculation are plotted in the following figures. Figure 9 and Figure 10 show the dose rate maps for the neutron CAAS detector response in a plan and elevation view that cuts through the center of Jezebel. Figure 11 and Figure 12 show the CAAS detector response 
to photons in the same planes as Figure 9 and Figure 10. As an aside, the sort of results plotted below would also be very useful when determining the initial evacuation zones following a criticality accident.

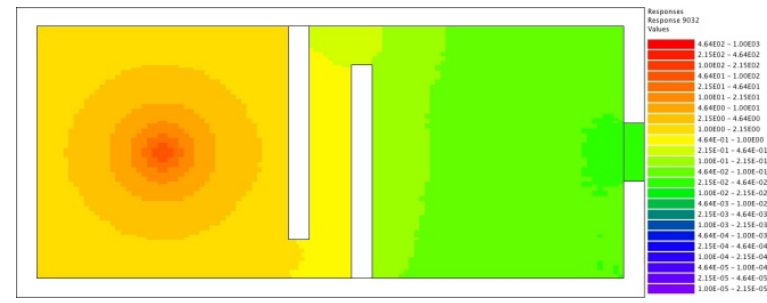

Figure 9. Plan view of forward calculated neutron air kerma rate map ( $\mathrm{z}=100, \mathrm{~Gy} / \mathrm{min})$.

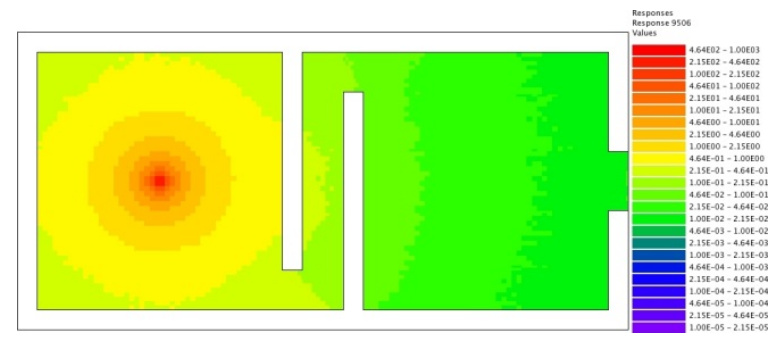

Figure 11. Plan view of forward calculated photon air kerma rate map (z=100, Gy/min).

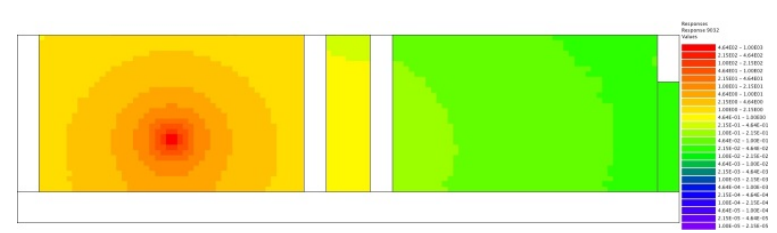

Figure 10. Elevation view of forward calculated neutron air kerma rate map ( $y=300, \mathrm{~Gy} / \mathrm{min})$.

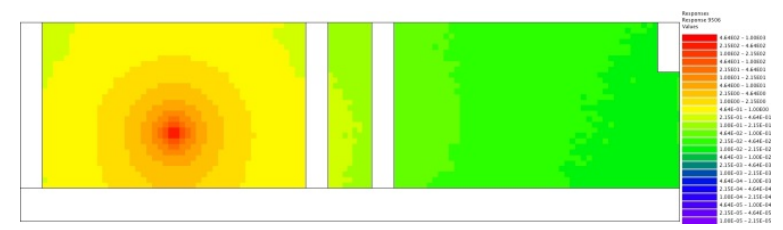

Figure 12. Elevation view of forward calculated photon air kerma rate map $(y=300, G y / m i n)$.

The minimum dose rate plotted in the four figures above is $1.6667 \times 10^{-5} \mathrm{~Gy} / \mathrm{min}$ (purple), which is the CAAS alarm set point assumed in Section 7. Only dose rate data in the air inside the block building is plotted, so the concrete walls and floor have no contours. Table 7 compares the results from Section 7.3, which used a region tally with a Jezebel point source to calculate the CAAS detector response, and the mesh tally results at the location of the center of the spherical CAAS detector used in Section 7 . The results compare well and all are statistically the same.

Table 7. Comparison between region tally and mesh tally dose rates at center of spherical CAAS (relative errors are at the 1- $\sigma$ level).

\begin{tabular}{|c|c|c|}
\hline Result & Tally Type & Dose Rate (Gy/min) \\
\hline SCALE Neutron & Region Tally & $3.6847 \mathrm{E}-02 \pm 0.47 \%$ \\
\hline SCALE Photon & Region Tally & $1.6957 \mathrm{E}-02 \pm 1.69 \%$ \\
\hline SCALE Neutron & Mesh Tally & $3.7505 \mathrm{E}-02 \pm 0.89 \%$ \\
\hline SCALE Photon & Mesh Tally & $1.6694 \mathrm{E}-02 \pm 2.03 \%$ \\
\hline MCNP Neutron & Region Tally & $3.6811 \mathrm{E}-02 \pm 0.44 \%$ \\
\hline MCNP Photon & Region Tally & $2.1418 \mathrm{E}-02 \pm 1.16 \%$ \\
\hline MCNP Neutron & Mesh Tally & $3.7494 \mathrm{E}-02 \pm 0.49 \%$ \\
\hline MCNP Photon & Mesh Tally & $2.1146 \mathrm{E}-02 \pm 0.66 \%$ \\
\hline
\end{tabular}

Plots like those in Figure 9 - Figure 12 could be created to help a practitioner determine where a CAAS detector would alarm due to a specific critical source modeled in the forward simulation. As many CAAS response functions as necessary could be convolved with the forward flux to evaluate any number of different CAAS detector types.

The source strength in this analysis was assumed to be $1 \times 10^{15}$ fissions per second, the same as Section 7 . This fission rate is so large that the critical accident is easily detected anywhere inside this block building, as long as the flux-to-dose-rate conversion factors are the ICRU kerma factors. The fission rate will be adjusted in the adjoint analysis, which is discussed next, so that it is harder to detect the critical assembly. 
The only results produced by an adjoint Denovo calculation in SCALE 6.1 are adjoint scalar fluxes. These adjoint scalar fluxes have to be convolved with the forward source energy distribution (Jezebel leakage spectra) to calculate the CAAS detector response. At the same time, any additional multiplication factors can be applied, such as the strength of the fission source or conversion to units of $\mathrm{Gy} / \mathrm{min}$. This process requires the use of the MAVRIC utility vsResp, which is one of several utilities developed since the release of SCALE 6.1. A complete description of vsResp and the other new MAVRIC utilities is provided in the appendices of the SCALE MAVRIC manual. The full input for vsResp is supplied in Appendix A.16. An important thing to remember is that flux-to-dose-rate conversion factors entered in vsResp should be entered in forward order, i.e., from high energy to low, starting with neutrons followed by photons.

To make the results produced by the adjoint Denovo calculation a little more interesting than those produced by the forward mesh tallies, the smallest detectable fission rates calculated by SCALE (Table 6) are considered. The fission rate applied to calculate the neutron CAAS detector response with the Denovo adjoint fluxes is $4.5 \times 10^{11}$ fissions per second. For the photon CAAS detector response, the fission rate is $9.9 \times 10^{11}$ fissions per second. These are both less than the smallest fission rate that can be detected by the CAAS detector shown in Figure 5, assuming the flux-to-dose-rate conversion factors used are the ICRU air kerma factors. Obviously, this means the spherical CAAS detector in Figure 5 should not provide full coverage for the entire block building due to these low fission rates, and this is shown in the figures below. Figure 13 and Figure 14 show the air kerma rate maps for a neutron CAAS detector response in a plan and elevation view that cuts through the center of Jezebel. Figure 15 and Figure 16 show the CAAS detector response to photons, cutting through the same planes.

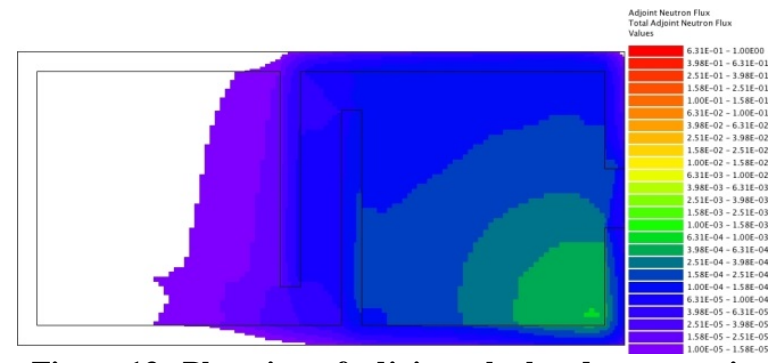

Figure 13. Plan view of adjoint calculated neutron air kerma rate map $(\mathrm{z}=100, \mathrm{~Gy} / \mathrm{min})$.

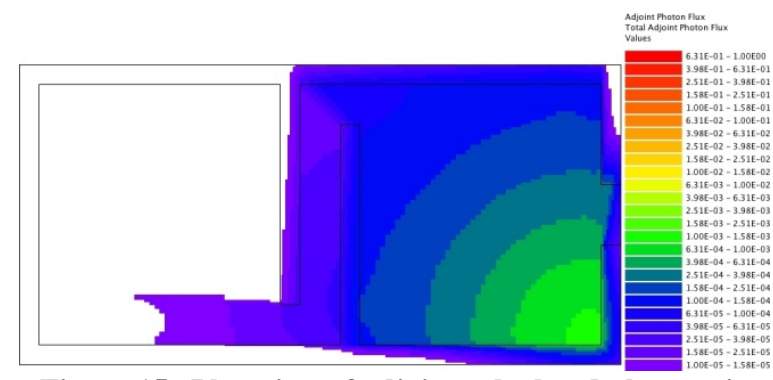

Figure 15. Plan view of adjoint calculated photon air kerma rate map $(\mathrm{z}=100, \mathrm{~Gy} / \mathrm{min})$.

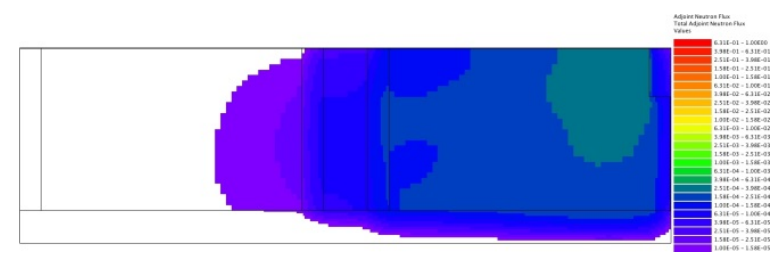

Figure 14. Elevation view of adjoint calculated neutron air kerma rate map ( $y=300, G y / m i n)$.

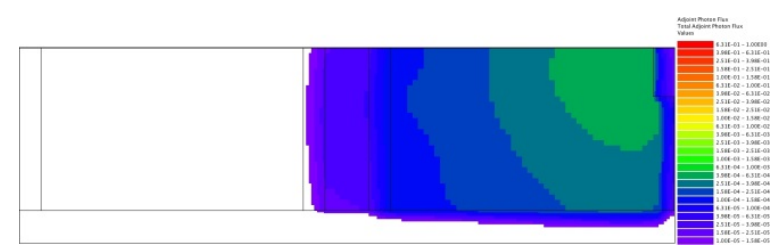

Figure 16. Elevation view of adjoint calculated photon air kerma rate map ( $y=300, G y / m i n)$.

The minimum dose rate plotted in the four figures above is $1.6667 \times 10^{-5} \mathrm{~Gy} / \mathrm{min}$ (purple), which is the CAAS alarm set point assumed in Section 7. Since the deterministic calculation solved for the adjoint flux inside the concrete walls and floor of the model, the dose rates in the concrete are shown (unlike Figure 9 - Figure 12). The white areas (no color contours) in the left room indicate where there is no CAAS coverage provided by the spherical detector in Figure 5. Data are available to plot the CAAS detector response in these white areas but have not been plotted to make it clear where there is no CAAS 
coverage. The CAAS detector responses appear to be under predicted, particularly the photon air kerma rates. This is due to the approximate nature of the Denovo adjoint model (course grid geometry and angular quadrature). A calculation with a finer geometry mesh and angular quadrature would produce a more accurate solution, which would result in color contour lines extending closer to the location of Jezebel, but still not cover the entire left room. Plots like Figure 13 - Figure 16 can be created to help a practitioner determine if a CAAS detector in a specific location with a specific set of flux-to-dose-rate conversion factors would alarm due to a critical assembly. Any number of fission source spectra and strengths could be used in this sort of analysis. As a reminder, the fission source has to be approximated as a point in order to apply the adjoint method with a mesh tally.

\section{CAAS PLACEMENT ANALYSIS STRATEGY AND EXAMPLES}

The previous sections have introduced the concepts needed to perform calculations to analyze the response of a CAAS detector to a critical assembly using a number of different methods. This section outlines a strategy to perform an analysis that will help determine the optimum placement of the minimum number of CAAS detectors using the methods already described in this guidance document. The discussion of this strategy is followed by an example that is complex enough to represent an actual facility, but not so complex that novice practitioners cannot follow along.

Determining the optimum placement of the minimum number of CAAS detectors that can detect a critical assembly anywhere in a large facility is a complex problem. Typically there are a target number of detectors that are desired to cover a given zone of a facility. A study to determine detector placement typically begins with some initial guess at the placement of the detectors, and is followed by either predictive calculations of accidents at specific locations or adjoint calculations using the detectors as sources.

Within a zone of a facility, there are an infinite number of potential accident sites. For any given placement of the detectors, the list of accident sites can be reduced to a finite number of locations that would be difficult for the detectors to detect. A further reduction in the number of accident locations can be made by determining whether the possible accident locations and scenarios are credible. Developing the initial placement of detectors and determining the list of difficult accident locations are based on the practitioner's experience.

If the number of accidents, $A$, is much less than the number of detectors, $D$, then forward simulations may be more convenient and less time-consuming. If $D$ is much less than $A$, then adjoint calculations may be more efficient. Forward calculations employing a mesh tally are useful if the accident type and location is fixed, but the CAAS detector type and location are unknown. Adjoint calculations employing a mesh tally are advantageous since they do not rely on a list of specific accidents, which may not have included every possible accident location. However, adjoint calculations require more information about the type and location of the CAAS detector.

Depending on the geometry of the problem, the number of detectors and the number of accident sites, different approaches to CAAS placement studies can be taken. These are summarized in Table 8 below. 
Table 8. Different approaches for CAAS detector placement studies.

\begin{tabular}{|c|c|c|c||c|c|c|c|}
\hline \multicolumn{4}{|c||}{ Gumber of detector locations = D } & \multicolumn{5}{c|}{ Number of accident Sites = A } \\
\hline \multicolumn{3}{|c|}{ Geometry } & \multicolumn{5}{c|}{ Approach } \\
\hline Comparison & A & D & Shielding & Number & Direction & Biasing & Tallies \\
\hline \hline A $<\mathrm{D}$ & small & small & sparse & 1 & forward & analog & standard tallies \\
\hline A $<\mathrm{D}$ & small & large & sparse & 2 & forward & analog & mesh tally \\
\hline A $<\mathrm{D}$ & small & small & dense & 3 & forward & CADIS & standard tallies \\
\hline A $<\mathrm{D}$ & small & large & dense & 4 & forward & FW-CADIS & mesh tally \\
\hline $\mathrm{D}<\mathrm{A}$ & small & small & sparse & 5 & adjoint & analog & standard tallies \\
\hline $\mathrm{D}<\mathrm{A}$ & large & small & sparse & 6 & adjoint & analog & mesh tally \\
\hline $\mathrm{D}<\mathrm{A}$ & small & small & dense & 7 & adjoint & CADIS & standard tallies \\
\hline $\mathrm{D}<\mathrm{A}$ & large & small & dense & 8 & adjoint & FW-CADIS & mesh tally \\
\hline
\end{tabular}

In Table 8 the Shielding column refers to how much attenuation there is between the accidents and detectors, which includes geometric attenuation (distance) and physical attenuation by shielding materials. The Direction column refers to whether the calculation is a forward or adjoint transport calculation. Biasing noted as analog is not truly analog, because implicit capture and weight cutoff with roulette are used. In this case, analog simply means no automated variance reduction like CADIS or FWCADIS are applied. Finally, standard tallies refer to any tallies other than mesh tallies, typically region (volume) or point detector tallies.

To test the different approaches to analyze CAAS detector placement, a simple example problem has been created. The problem consists of a fuel storage room filled with 18 storage racks. Each rack consists of an array of 80 double-sided steel storage bins, each side containing a cuboid of about $21 \mathrm{~kg}$ of natural $\mathrm{UO}_{2}$. Each storage bin is a cube of $30.48 \mathrm{~cm}$. The basic geometry is shown in Figure 17 - Figure 19.

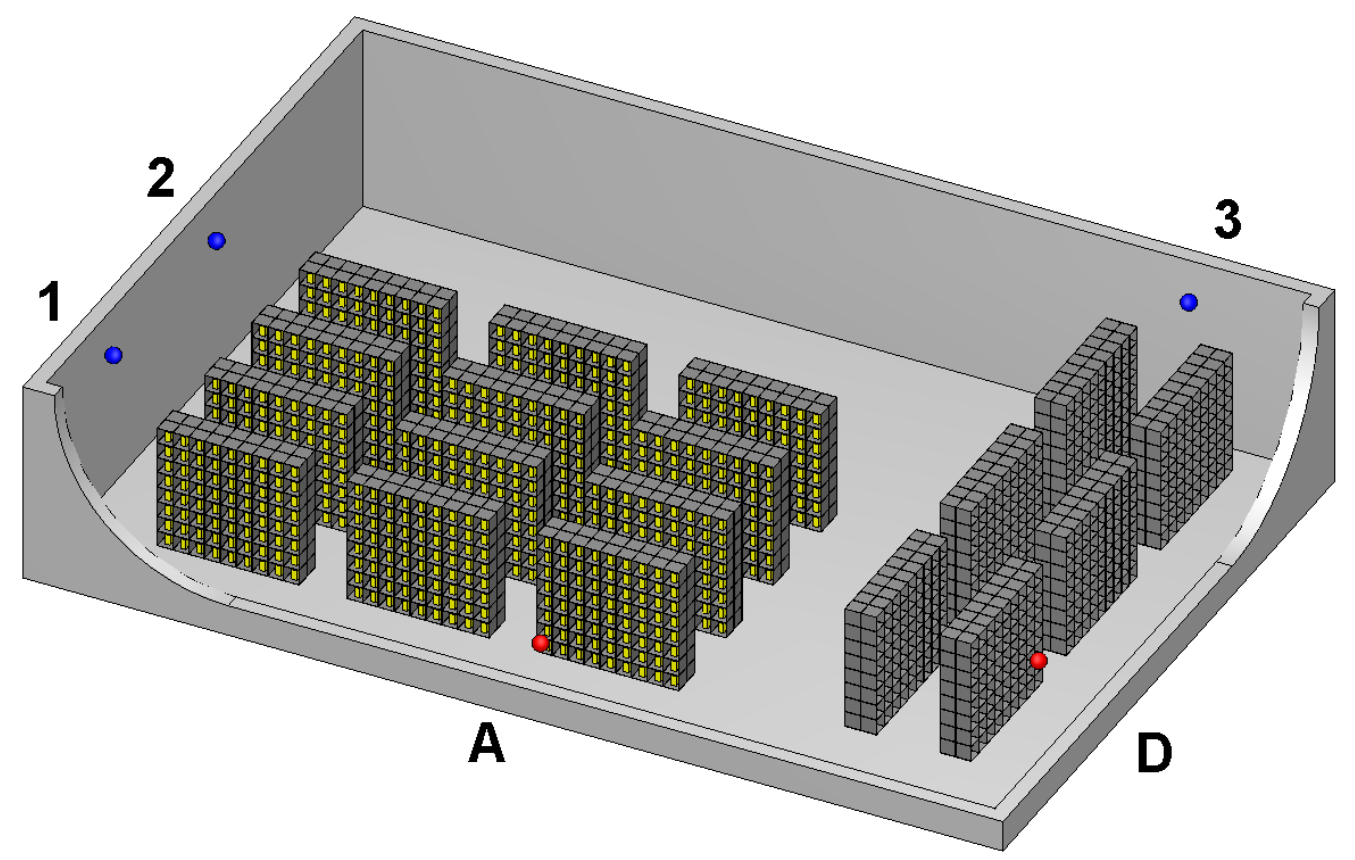

Figure 17. The storage room (ceiling and walls removed for visualization) showing the detector locations (blue, $3 \mathrm{~m}$ above floor) and some accident locations (red). Accident locations $B$ and $C$ are between the racks and not visible in this view. 


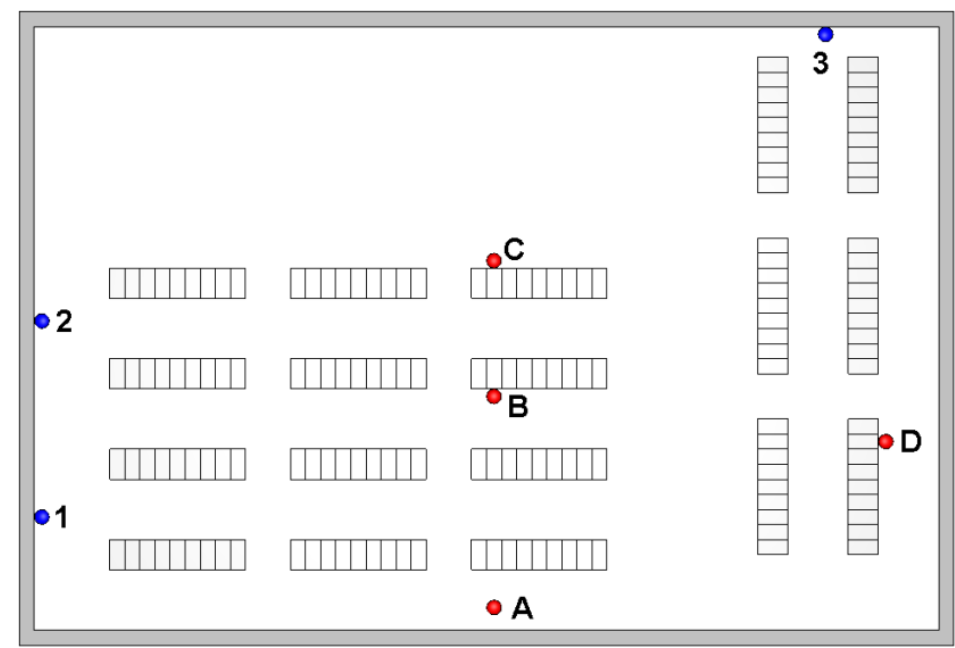

Figure 18. Overhead view of the storage room showing three detector locations (blue) and four accident sites (red).

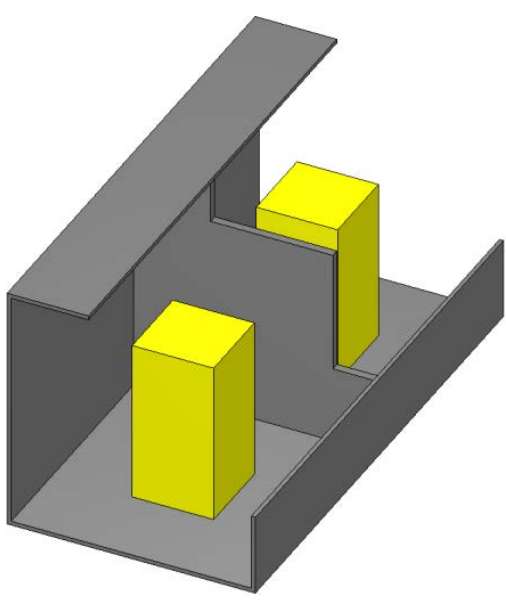

Figure 19. Close-up view of the double-sided storage bin, with sides removed.

For the purposes of this example, it is assumed that the minimum credible accident produces $2.5 \times 10^{15}$ neutrons (no photons), which can be modeled as a point isotropic source with an energy distribution of a generic ${ }^{235} \mathrm{U}$ Watt spectrum in a single burst. The CAAS detector will only respond to photons, and the alarm set point for this example is assumed to be a photon dose of $0.150 \mathrm{rem}$. It is important to note that this is an equivalent dose, not absorbed dose in air; therefore, different flux-to-dose-rate conversion factors must be applied. In this case, the 1977 ANSI/ANS photon flux-to-dose-rate conversion factors [16] are applied. The remainder of Section 9 will use some of the different approaches of CAAS detector placement analysis listed in Table 8 to calculate the CAAS detector response for the example that has just been introduced. For brevity, MCNP will not be applied with every approach. SCALE will be applied with and without automated variance reduction and MCNP will be applied only with automated variance reduction.

\subsection{FORWARD PLACEMENT ANALYSIS}

Note that MAVRIC normally calculates dose rates (rem/hr) from continuous sources (neutron/sec). Since this study models a single burst, the normal flux-to-dose-rate conversion factors ( $\mathrm{rem} / \mathrm{hr}) /(/ \mathrm{cm} 2 / \mathrm{sec})$ are multiplied by $1 \mathrm{hr} / 3600 \mathrm{sec}$. This way, the tally results will be doses and have units of rem.

\subsubsection{Approach 1: Forward Simulation, Analog MC, Standard Tallies}

In this approach, a forward simulation is performed for each of the accident locations, with region tallies for each detector. This approach works well for a small number of potential accident sites, a small number of detector locations, and a sparse geometry. The four simulations, one for each source, calculated the dose at all three detector locations, which are shown in the Analog column of Table 9. The full SCALE input for these calculations are in Appendix A.17. 
Table 9. Results for simple region tallies using analog and CADIS methods (highlighted doses are above the 0.150 rem alarm set point - relative errors are at the 1- $\sigma$ level).

\begin{tabular}{|c|c||c|c||c|c||c|c|}
\hline \multirow{2}{*}{ Source } & Detector & $\begin{array}{c}\text { SCALE Analog } \\
\text { Gamma Dose (rem) }\end{array}$ & $\begin{array}{c}\text { Analog } \\
\text { FOM }\end{array}$ & $\begin{array}{c}\text { SCALE CADIS } \\
\text { Gamma Dose (rem) }\end{array}$ & $\begin{array}{c}\text { CADIS } \\
\text { FOM }\end{array}$ & $\begin{array}{c}\text { MCNP CADIS } \\
\text { Gamma Dose (rem) }\end{array}$ & $\begin{array}{c}\text { MCNP } \\
\text { FOM }\end{array}$ \\
\hline \hline \multirow{3}{*}{$\mathrm{A}$} & 1 & $1.757 \mathrm{E}-01 \pm 3.00 \%$ & 1.84 & $1.802 \mathrm{E}-01 \pm 2.78 \%$ & 6.79 & $2.439 \mathrm{E}-01 \pm 0.41 \%$ & 16.5 \\
\cline { 2 - 9 } & 2 & $1.092 \mathrm{E}-01 \pm 3.79 \%$ & 1.15 & $1.083 \mathrm{E}-01 \pm 2.48 \%$ & 8.54 & $1.535 \mathrm{E}-01 \pm 0.48 \%$ & 10.5 \\
\cline { 2 - 9 } & 3 & $9.677 \mathrm{E}-02 \pm 4.07 \%$ & 1.00 & $9.285 \mathrm{E}-02 \pm 3.46 \%$ & 4.32 & $1.239 \mathrm{E}-01 \pm 0.54 \%$ & 7.85 \\
\hline \hline \multirow{3}{*}{$\mathrm{B}$} & 1 & $1.858 \mathrm{E}-01 \pm 3.11 \%$ & 1.70 & $1.799 \mathrm{E}-01 \pm 2.43 \%$ & 8.90 & $2.467 \mathrm{E}-01 \pm 0.41 \%$ & 15.1 \\
\cline { 2 - 9 } & 2 & $1.777 \mathrm{E}-01 \pm 3.17 \%$ & 1.63 & $1.790 \mathrm{E}-01 \pm 2.43 \%$ & 8.70 & $2.392 \mathrm{E}-01 \pm 0.47 \%$ & 9.90 \\
\cline { 2 - 9 } & 3 & $1.441 \mathrm{E}-01 \pm 3.50 \%$ & 1.34 & $1.543 \mathrm{E}-01 \pm 3.26 \%$ & 4.85 & $2.048 \mathrm{E}-01 \pm 0.52 \%$ & 9.22 \\
\hline \hline & 1 & $1.061 \mathrm{E}-01 \pm 3.83 \%$ & 1.12 & $1.168 \mathrm{E}-01 \pm 3.31 \%$ & 4.79 & $1.474 \mathrm{E}-01 \pm 0.48 \%$ & 10.3 \\
\cline { 2 - 9 } & 2 & $1.961 \mathrm{E}-01 \pm 2.91 \%$ & 1.95 & $1.904 \mathrm{E}-01 \pm 2.40 \%$ & 8.96 & $2.674 \mathrm{E}-01 \pm 0.44 \%$ & 14.7 \\
\cline { 2 - 9 } & 3 & $2.976 \mathrm{E}-01 \pm 2.31 \%$ & 3.08 & $2.901 \mathrm{E}-01 \pm 2.59 \%$ & 7.65 & $3.947 \mathrm{E}-01 \pm 0.46 \%$ & 19.5 \\
\hline \hline \multirow{3}{*}{$\mathrm{D}$} & 1 & $5.347 \mathrm{E}-02 \pm 5.70 \%$ & 0.51 & $4.665 \mathrm{E}-02 \pm 4.08 \%$ & 3.14 & $6.503 \mathrm{E}-02 \pm 1.28 \%$ & 1.25 \\
\cline { 2 - 9 } & 2 & $5.714 \mathrm{E}-02 \pm 5.25 \%$ & 0.60 & $5.656 \mathrm{E}-02 \pm 3.97 \%$ & 3.23 & $7.393 \mathrm{E}-02 \pm 1.30 \%$ & 1.22 \\
\cline { 2 - 9 } & 3 & $2.440 \mathrm{E}-01 \pm 2.56 \%$ & 2.54 & $2.430 \mathrm{E}-01 \pm 1.97 \%$ & 13.30 & $3.329 \mathrm{E}-01 \pm 0.52 \%$ & 10.6 \\
\hline
\end{tabular}

\subsubsection{Approach 2: Forward Simulation, Analog MC, Mesh Tally}

For a small number of accident sites and a large number of detector locations (or unspecified detector locations), this approach calculates a mesh tally over the entire model, which can be used to show where detectors would alarm for a given accident. Four gamma dose mesh tallies, one for each source, have been generated using SCALE and are shown in Figure 20 - Figure 23 for the plane $(z=290 \mathrm{~cm})$ that includes the detector locations. Note that the values in the mesh tally for the detector locations are statistically the same as the analog results in Table 9 . The dose contour for $0.150 \mathrm{rem}$, the alarm set point, is between the two light green colors ( $(v)$. Also note that the default label 'rem/hour' is wrong since these values are actually 'rem' (see the note at the beginning of Section 9.1). The full SCALE inputs for these calculations are in Appendix A.18.

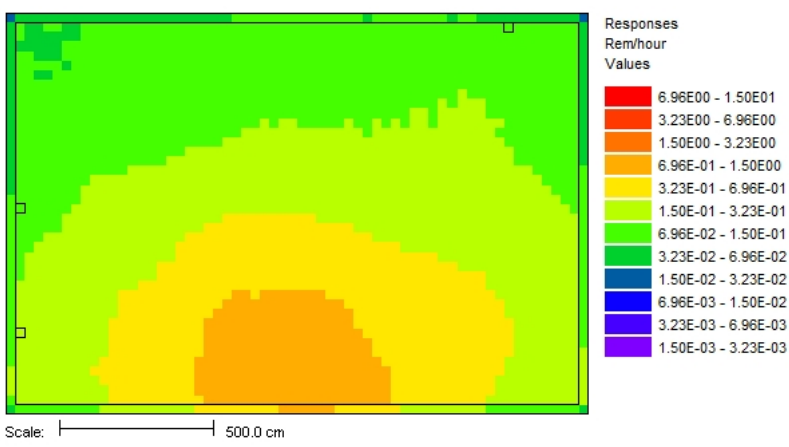

Figure 20. Gamma dose mesh tally from source A (forward, analog).

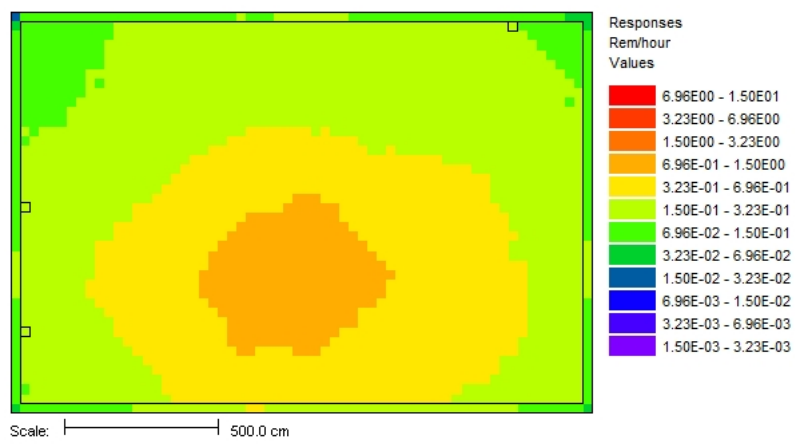

Figure 21. Gamma dose mesh tally from source B (forward, analog). 


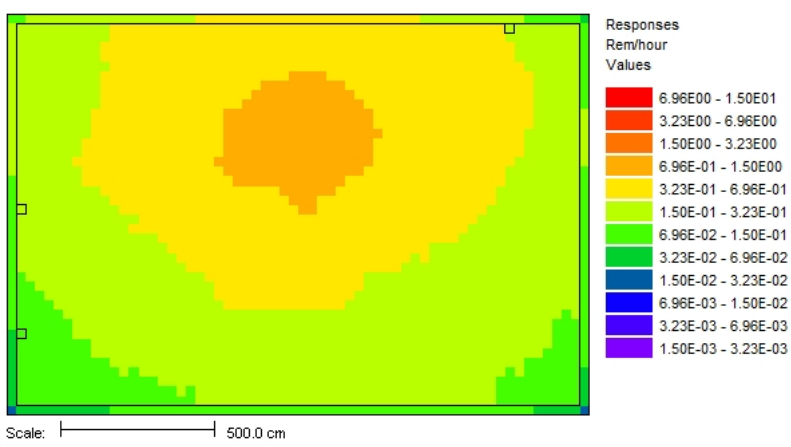

Figure 22. Gamma dose mesh tally from source C (forward, analog).

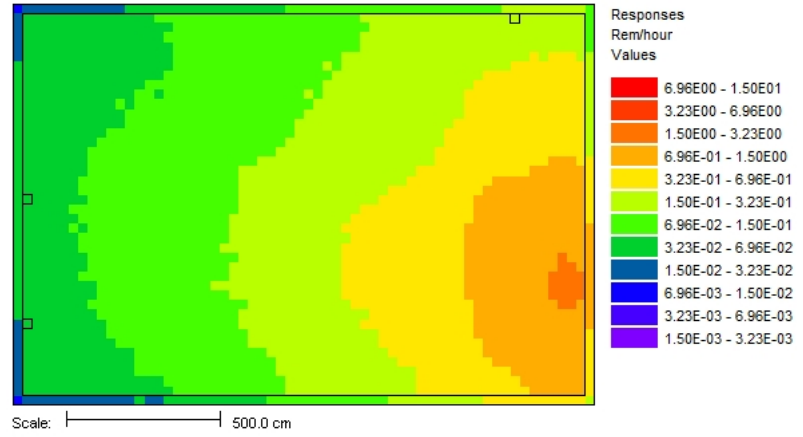

Figure 23. Gamma dose mesh tally from source D (forward, analog).

\subsubsection{Approach 3: Forward Simulation, CADIS, Standard Tallies}

For dense geometries, CADIS may be required to accelerate the Monte Carlo calculation of CAAS detector responses. For a small number of potential accident sites, $A$, and a small number of detector locations, $D$, this approach requires one simulation for each combination of $A$ and $D$. The results, similar to those using the analog approach, are also shown in Table 9 in the SCALE CADIS column. MCNP computational results for this approach are also included in Table 9 in the MCNP CADIS column. The CADIS biasing parameters for MCNP have been generated using ADVANTG. One will notice that the MCNP CADIS results are different from the SCALE results. The SCALE results are between 20 and 30 percent lower than the MCNP results. An explanation for the difference between MCNP and SCALE is provided in Section 9.1.4. However, note that this difference is not completely due to the difference in photon transport physics between MCNP and SCALE, which has been discussed in previous sections. The full SCALE and MCNP inputs for this approach are included in Appendix A.19.a and A.19.b, respectively.

Even for this simple example, the CADIS calculations perform more efficiently than the analog calculations. The FOM for each CADIS result in Table 9 is larger than the equivalent analog result. The total time to compute all 12 accident/detector combinations with 12 input files using SCALE CADIS is roughly the same as the total time for the four SCALE analog inputs. Each CADIS calculation converged the single optimized CAAS detector response more efficiently, but the limitation of optimizing one detector response per calculation caused the total run time between the 4 analog and 12 CADIS calculations to be about the same.

The SCALE CADIS and SCALE analog dose values in Table 9 agree well. Only source B/detector 3 gives different mean results as far as the alarm set point is concerned. However, the SCALE analog and SCALE CADIS values are statistically the same on the 95\% confidence interval (2 sigma). Therefore, statistically speaking, the analog source B/detector 3 result in Table 9 should also be highlighted to indicate exceeding the alarm set point. While this is a perfectly valid argument that proves the results are statistically the same, this argument does not produce conservative results. In CAAS analyses using Monte Carlo calculations, a conservative result is one in which the mean is at least two or three standard deviations above the alarm set point.

\subsubsection{Approach 4: Forward Simulation, FW-CADIS, Mesh Tally}

For dense geometries and large numbers of detectors or unknown detector locations, this approach uses the FW-CADIS method to compute a mesh tally over the entire facility, with biasing parameters designed to obtain uniform relative uncertainties in the gamma dose for both high- and low-dose areas. Using this approach, a gamma dose mesh tally has been calculated for each source location using SCALE, which is 
shown in Figure 24 - Figure 27 for the plane $(z=290 \mathrm{~cm})$ that includes the detector locations. The following four figures are very similar to the four figures in Section 9.1.2, as expected. The different variance reduction techniques do not impact the final answer, if applied properly, which is evident by the SCALE results for standard tallies in Table 9. The full SCALE and MCNP inputs for this approach are included in Appendices A.20.a and A.20.b, respectively. The FW-CADIS biasing parameters have been generated for MCNP with the ADVANTG code.
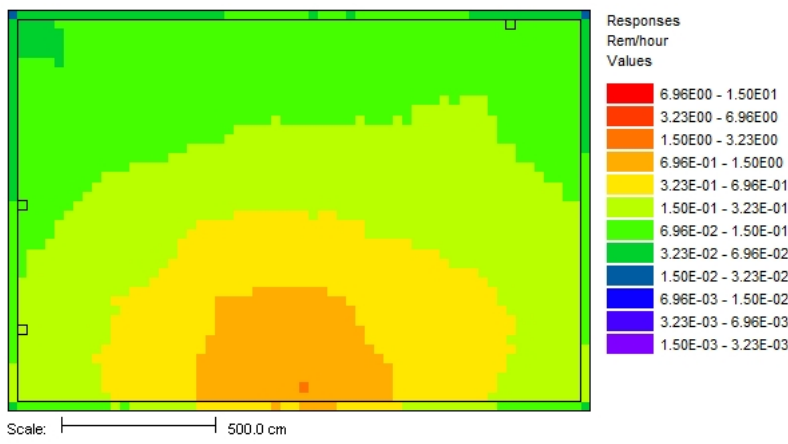

Figure 24. Gamma dose mesh tally from source A (forward, FW-CADIS).

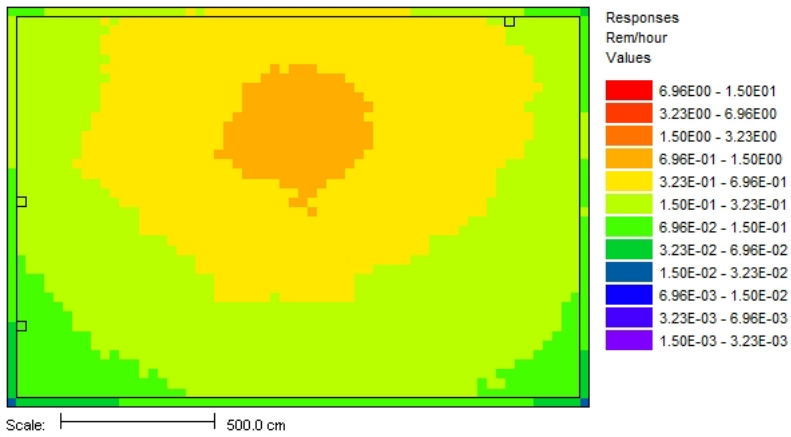

Figure 26. Gamma dose mesh tally from source C (forward, FW-CADIS).

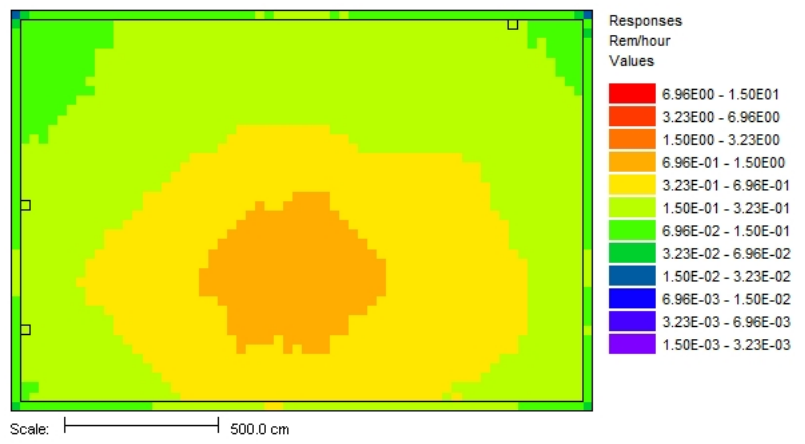

Figure 25. Gamma dose mesh tally from source B (forward, FW-CADIS).

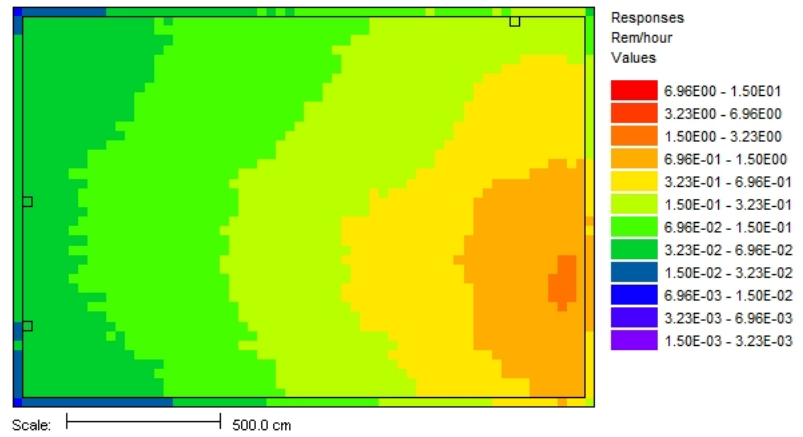

Figure 27. Gamma dose mesh tally from source D (forward, FW-CADIS).

Plots similar to those in Figure 24 - Figure 27 can be generated with the MCNP mesh tally results. To make a more exact comparison between the SCALE and MCNP mesh tally results for this approach, the CAAS detector response at the detector locations in Figure 18 from the SCALE and MCNP mesh tallies are compared in Table 10.

Table 10. Comparison of CAAS detector responses (rem) calculated with FW-

CADIS and mesh tallies (errors are at the 1- $\sigma$ level).

\begin{tabular}{|c|c|c|c|c|}
\hline Source & Detector & $\begin{array}{c}\text { SCALE } \\
\text { FW-CADIS }\end{array}$ & $\begin{array}{c}\text { MCNP } \\
\text { FW-CADIS }\end{array}$ & $\begin{array}{c}\text { Ratio: } \\
\text { SCALE / MCNP }\end{array}$ \\
\hline \hline \multirow{3}{*}{$\mathrm{A}$} & 1 & $1.807 \mathrm{E}-1 \pm 2.55 \%$ & $2.401 \mathrm{E}-1 \pm 0.49 \%$ & $0.75 \pm 0.02$ \\
\cline { 2 - 5 } & 2 & $1.064 \mathrm{E}-1 \pm 2.00 \%$ & $1.501 \mathrm{E}-1 \pm 0.46 \%$ & $0.71 \pm 0.01$ \\
\cline { 2 - 5 } & 3 & $9.547 \mathrm{E}-2 \pm 2.45 \%$ & $1.221 \mathrm{E}-1 \pm 0.48 \%$ & $0.78 \pm 0.02$ \\
\hline \hline \multirow{3}{*}{$\mathrm{B}$} & 1 & $1.906 \mathrm{E}-1 \pm 2.38 \%$ & $2.433 \mathrm{E}-1 \pm 0.51 \%$ & $0.78 \pm 0.02$ \\
\cline { 2 - 5 } & 2 & $1.767 \mathrm{E}-1 \pm 2.26 \%$ & $2.345 \mathrm{E}-1 \pm 0.48 \%$ & $0.75 \pm 0.02$ \\
\cline { 2 - 5 } & 3 & $1.587 \mathrm{E}-1 \pm 2.46 \%$ & $2.008 \mathrm{E}-1 \pm 0.52 \%$ & $0.79 \pm 0.02$ \\
\hline \hline & 1 & $1.123 \mathrm{E}-1 \pm 2.24 \%$ & $1.462 \mathrm{E}-1 \pm 0.47 \%$ & $0.77 \pm 0.02$ \\
\cline { 2 - 5 } & 2 & $1.934 \mathrm{E}-1 \pm 2.25 \%$ & $2.637 \mathrm{E}-1 \pm 0.45 \%$ & $0.73 \pm 0.02$ \\
\cline { 2 - 5 } & 3 & $2.985 \mathrm{E}-1 \pm 2.36 \%$ & $3.894 \mathrm{E}-1 \pm 0.50 \%$ & $0.77 \pm 0.02$ \\
\hline \hline & 1 & $4.782 \mathrm{E}-2 \pm 3.06 \%$ & $6.288 \mathrm{E}-2 \pm 0.68 \%$ & $0.76 \pm 0.02$ \\
\cline { 2 - 5 } & 2 & $5.722 \mathrm{E}-2 \pm 4.22 \%$ & $7.168 \mathrm{E}-2 \pm 0.83 \%$ & $0.80 \pm 0.03$ \\
\cline { 2 - 5 } & 3 & $2.435 \mathrm{E}-1 \pm 2.15 \%$ & $3.252 \mathrm{E}-1 \pm 0.52 \%$ & $0.75 \pm 0.02$ \\
\hline
\end{tabular}


As noted in Table 9, the SCALE CAAS detector responses are between 20 and 30 percent lower than the MCNP calculated CAAS detector responses in Table 10. Turning off the additional MCNP photon physics (lower cutoff energy and thick target bremsstrahlung model) did not significantly affect the difference between MCNP and SCALE. In fact, the MCNP results presented in Table 10 have this additional transport physics turned off (the additional photon transport physics is turned on in Table 9). Investigations have concluded that these differences are primarily due to the multi-group representation of the photon cross sections used in the SCALE calculations. The multi-group library used in the calculations is the 200-neutron group/47-gamma group SCALE library based on ENDF/B-VII.0. The problem with the multi-group photon cross sections arises from the fact that the source in this problem is a neutron only source, so there are no source photons born with a large distribution of energies. Instead, most of the photons are created in this problem via inelastic scattering or neutron capture, which tend to produce photons with discrete energies. The 47 energy groups in this cross-section library do not provide enough resolution to accurately model these discrete photons. In the presence of a photon continuum, these cross sections perform very well and this issue is not as prevalent. A few of the results in Table 10 have been recomputed using a continuous-energy version of MAVRIC/Monaco in the beta version of SCALE 6.2, which produced results that were between 6 and 12 percent lower than MCNP.

The standard FW-CADIS method is probably not desired for CAAS detector placement analysis because the method attempts to provide low relative uncertainties, even in areas below the CAAS alarm set point. For CAAS detector placement studies, the practitioner's area of interest may only be the area above the minimum dose that will trigger an alarm. SCALE 6.2 will contain keywords within the normal FWCADIS input for the importance map block to reduce the area of mesh tally optimization to those areas that are above some minimum estimated response, below some maximum estimated response, or both.

For SCALE 6.1, a utility can be used to process the result of the forward Denovo calculation, keeping only those areas where the estimated dose is above the alarm set point. Mesh tallies using this procedure should then be optimized to compute dose in only the area where the forward estimate of dose was above the alarm set point. For the four source locations, the mesh tallies in the plane of the CAAS detectors using this modified approach are shown in Figure 28 - Figure 31. Note that the areas above the 0.150 rem minimum dose look fairly close to the mesh tallies using the standard FW-CADIS approach. For areas below the 0.150 rem minimum dose, there is more statistical noise present than in the corresponding mesh tallies from the standard FW-CADIS. This is more obvious for source D.

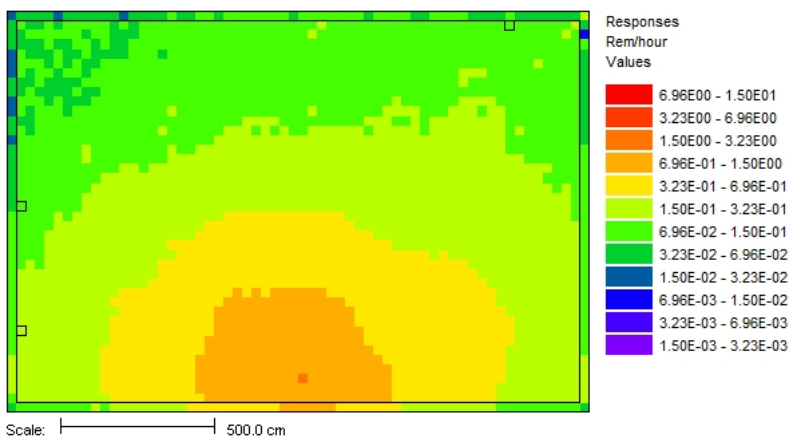

Figure 28. Gamma dose mesh tally from source A (forward, modified FW-CADIS).

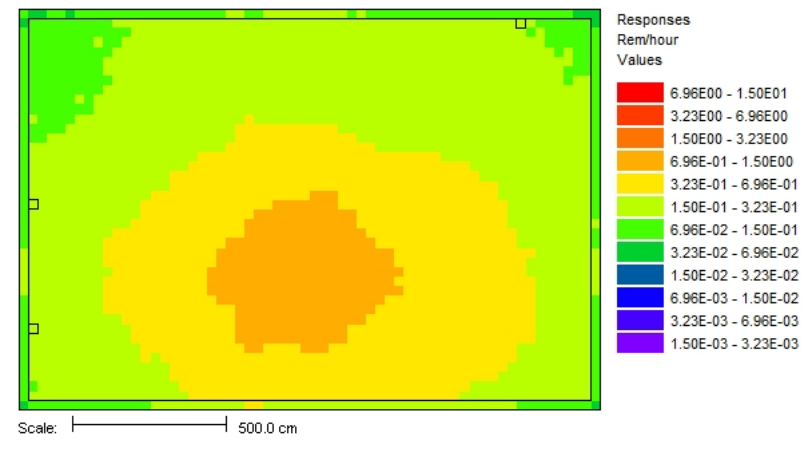

Figure 29. Gamma dose mesh tally from source B (forward, modified FW-CADIS). 


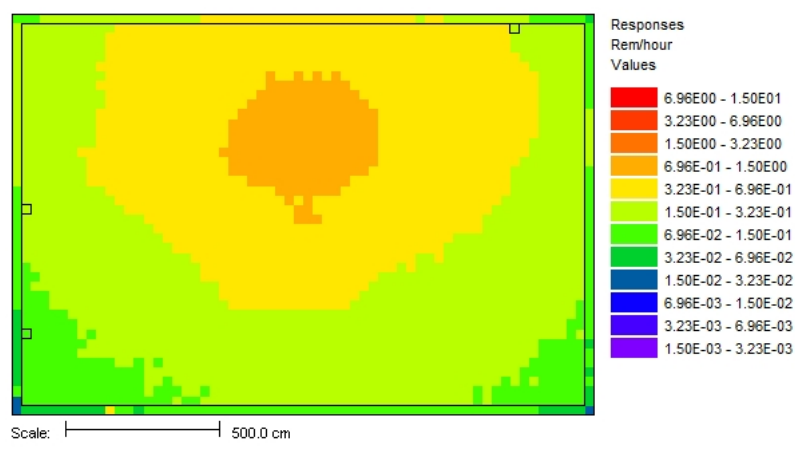

Figure 30. Gamma dose mesh tally from source C (forward, modified FW-CADIS).

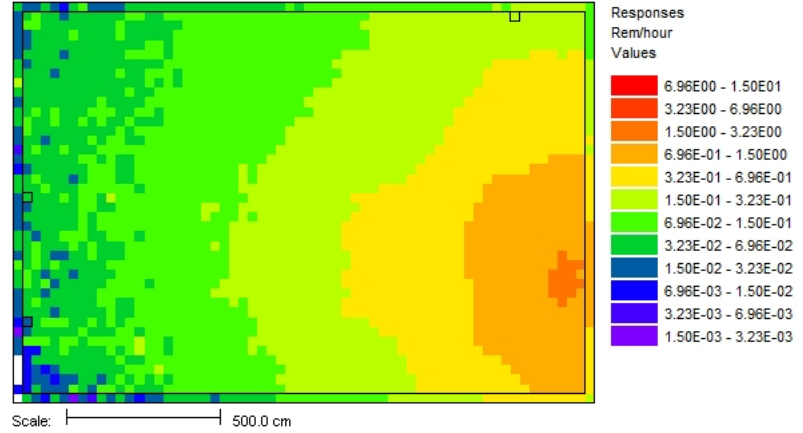

Figure 31. Gamma dose mesh tally from source D (forward, modified FW-CADIS).

This modified approach is more efficient than simply trying to optimize the mesh tally over the entire facility. For areas that lie outside of the area of optimization (which could include some detector locations), the mesh tally results of dose may be under-estimated and cannot be relied upon. This is what should happen; the Monte Carlo calculation should focus on those areas of the problem where the dose is above the CAAS alarm set point.

\subsubsection{Detector Placement using Forward Approaches}

With either of the forward approaches (approaches 2 and 4) that result in dose mesh tally maps, areas where detectors could see multiple accidents can be determined by filtering and adding the dose maps. SCALE 6.2 will include new MAVRIC utilities that perform the mesh tally filtering and adding operations described next. Consider the dose maps computed for each accident site shown in Figure 24 Figure 27. Each dose map has been filtered to show where a detector would alarm or not. These filtered dose maps are shown in Figure 32 - Figure 35 for the plane containing the CAAS detectors, with the red color indicating that a detector would alarm by an accident at the particular site and purple indicating that a detector would not alarm. Summing these new alarm/not alarm plots creates the plot in Figure 36, which shows for any given location how many of the four accidents could be seen at that location. This strategy is very useful in determining detector placement if there are only a few credible accident locations.

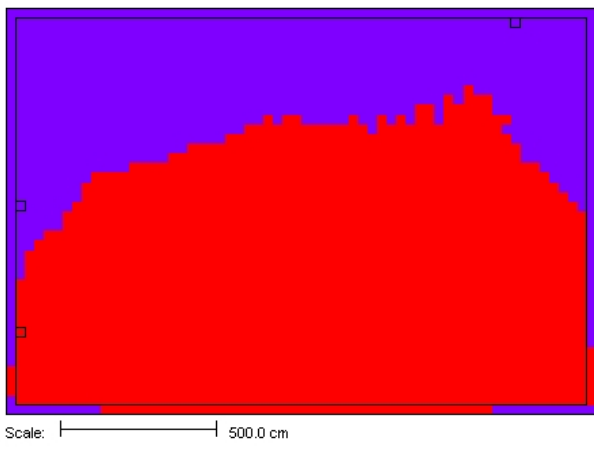

Figure 32. Areas that would alarm for accident site A.

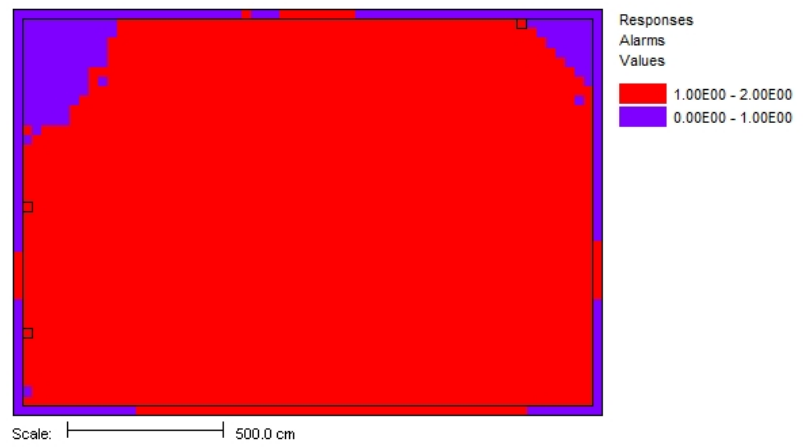

Figure 33. Areas that would alarm for accident site B. 


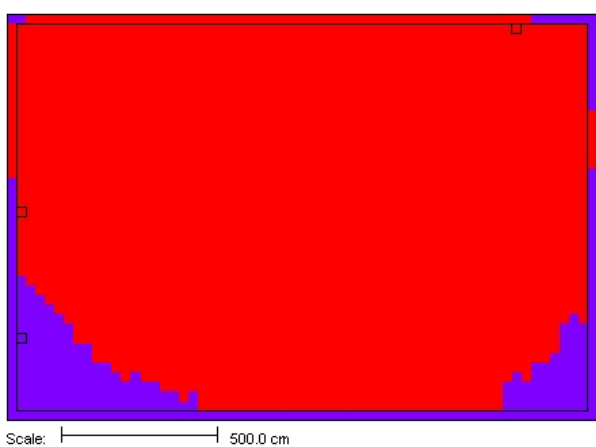

Figure 34. Areas that would alarm for accident site $C$.

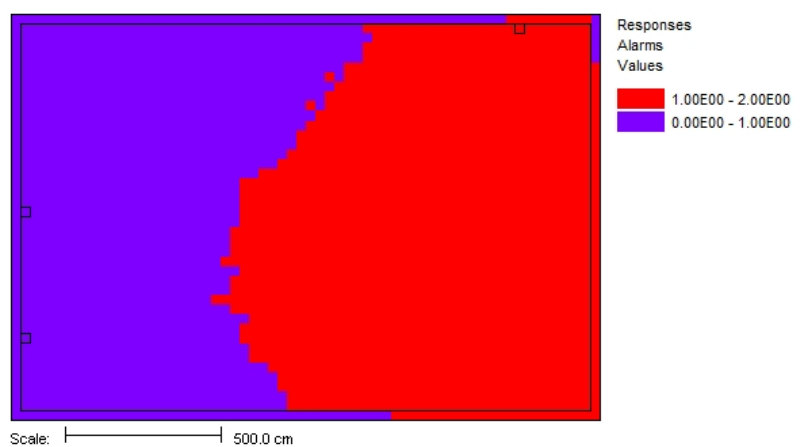

Figure 35. Areas that would alarm for accident site D.

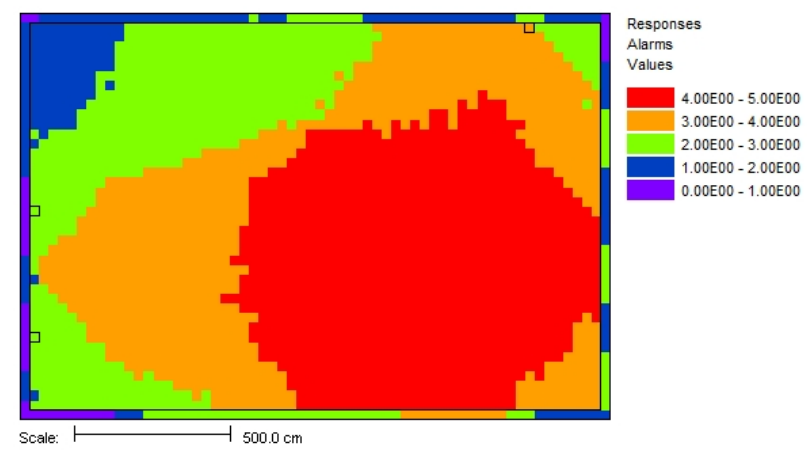

Figure 36. The number of accidents that can be seen from any given position at $\mathrm{z}=290 \mathrm{~cm}$.

\subsection{ADJOINT PLACEMENT ANALYSIS}

Monaco is able to perform adjoint neutron transport in the development version of SCALE, but photon transport has not been fully implemented. If this capability were added to SCALE, the four adjoint approaches listed in Table 9 would be available for performing CAAS placement studies with MAVRIC/Monaco. In Section 9.2.5, the Denovo deterministic transport code will be used as a surrogate for Monaco to apply the adjoint mesh tally approaches.

MCNP can perform neutron and photon adjoint transport in multi-group mode. However, as mentioned in Section 8.4, MCNP5 multi-group adjoint transport and mesh tallies are incompatible, so MCNP is only available for the adjoint approaches using standard tallies. Furthermore, ADVANTG is not capable of generating biasing parameters for MCNP adjoint transport calculations; therefore, MCNP5 will be applied only to approach 5.

\subsubsection{Approach 5: Adjoint Simulation, Analog MC, Standard Tallies}

One adjoint simulation would be performed for each detector location. The adjoint source would be the detector response function emitted from the detector location. A standard tally would be made at each credible accident location using the accident spectrum as the adjoint tally response function. The results of the tallies would have units of rem and a value greater than 0.150 rem would indicate that the specific detector/accident location would result in an alarm. The example defined in Figure 18 is evaluated using this approach. Some important details about adjoint transport calculations and MCNP adjoint calculations in general are discussed in Appendix $\mathrm{C}$ to provide a little more background. Appendix $\mathrm{C}$ also contains additional details about the MCNP adjoint input used for this example. Further details about multi-group adjoint MCNP calculations can be found in Reference 17. 
The easiest way to verify that one has correctly setup and run an adjoint calculation is to compare the results to an equivalent forward calculation. Obviously, this is not always practical and can be viewed as a waste of time and resources for projects with limited resources, but for this example the adjoint computational results will be compared to the forward computational results. This example has already been evaluated with MCNP in Section 9.1.3. However, in Section 9.1.3, continuous-energy cross-section data have been used in the forward transport calculation. Also, the source particle energy distribution and flux-to-dose-rate conversion factors used the SCALE 200 neutron and 47 photon group structures, and the CADIS variance reduction method was applied. As a result, the forward results calculated in Section 9.1.3 are not likely to compare well with the adjoint results using multi-group cross sections. Therefore, to begin this example, the forward calculation performed in Section 9.1.3 will be repeated with the MCNP MGXSNP multi-group cross sections, the source particle energy distribution and flux-to-dose-rate conversion factors will be collapsed to the MGXSNP group structure (details in Appendix C), and no automated variance reduction techniques will be applied. Using the same cross sections and group structure for the input data will guarantee that the forward and adjoint results statistically agree if the input files have been setup correctly. The MCNP input files used in Sections 9.1.3 and 9.1.4 (found in Appendices A.19.b and A.20.b) will be used as starting points for this example problem. The complete forward multi-group MCNP input files for this section are in Appendix A.21 with the adjoint multi-group input files in Appendix A.22.

In the initial step, four forward multi-group MCNP input files are created similar to those in A.20.b, one input per accident location (source). The region tallies in A.19.b replace the mesh tallies in A.20.b. However, unlike A.19.b, each source input file will contain three region tallies, one for each CAAS detector location in Figure 18, instead of a single region tally. Also, the group structure of the forward source particle energy distribution and forward flux-to-dose-rate conversion factors match that of the MGXSNP cross sections. Since this is an analog adjoint Monte Carlo simulation, the weight window parameter (WWP) and source biasing (SB1) cards are removed. Finally, the MGOPT card (data cards material specification) is added to select a forward transport calculation with the coupled MGXSNP cross-section data, and the material definitions are modified to match the elements / isotopes available in the MCXSNP cross-section data. In Table 11, the CAAS detector responses calculated by the forward multi-group simulations are compared to the forward continuous energy simulation from Section 9.1.3 (Table 9). One can see that the agreement between the continuous-energy and multi-group forward calculated detector responses, while not perfect, is fairly reasonable. The multi-group results are all overestimated compared to the continuous-energy results, but by less than $15 \%$. 
Table 11. Results of simple region tallies from forward and adjoint MCNP simulations (highlighted doses are above the 0.150 rem alarm set point - errors are at the 1- $\sigma$ level).

\begin{tabular}{|c|c|c|c|c|c|c|}
\hline Source & Detector & $\begin{array}{c}\text { MCNP Forward } \\
\text { CE CADIS } \\
\text { Gamma Dose (rem) }\end{array}$ & $\begin{array}{c}\text { MCNP Forward } \\
\text { MG Analog } \\
\text { Gamma Dose (rem) }\end{array}$ & $\begin{array}{l}\text { Ratio: } \\
\text { Forward } \\
\text { MG / CE }\end{array}$ & $\begin{array}{c}\text { MCNP Adjoint } \\
\text { MG Analog } \\
\text { Gamma Dose (rem) }\end{array}$ & $\begin{array}{c}\text { Ratio: } \\
\text { MG } \\
\text { For / Adj }\end{array}$ \\
\hline \multirow{3}{*}{ A } & 1 & $2.439 \mathrm{E}-01 \pm 0.41 \%$ & $2.723 \mathrm{E}-01 \pm 0.45 \%$ & $1.12 \pm 0.01$ & $2.732 \mathrm{E}-01 \pm 0.24 \%$ & $1.00 \pm 0.01$ \\
\hline & 2 & $1.535 \mathrm{E}-01 \pm 0.48 \%$ & $1.643 \mathrm{E}-01 \pm 0.57 \%$ & $1.07 \pm 0.01$ & $1.641 \mathrm{E}-01 \pm 0.31 \%$ & $1.00 \pm 0.01$ \\
\hline & 3 & $1.239 \mathrm{E}-01 \pm 0.54 \%$ & $1.323 \mathrm{E}-01 \pm 0.63 \%$ & $1.07 \pm 0.01$ & $1.321 \mathrm{E}-01 \pm 0.33 \%$ & $1.00 \pm 0.01$ \\
\hline \multirow{3}{*}{ B } & 1 & $2.467 \mathrm{E}-01 \pm 0.41 \%$ & $2.712 \mathrm{E}-01 \pm 0.45 \%$ & $1.10 \pm 0.01$ & $2.708 \mathrm{E}-01 \pm 0.28 \%$ & $1.00 \pm 0.01$ \\
\hline & 2 & $2.392 \mathrm{E}-01 \pm 0.47 \%$ & $2.633 \mathrm{E}-01 \pm 0.45 \%$ & $1.10 \pm 0.01$ & $2.650 \mathrm{E}-01 \pm 0.30 \%$ & $0.99 \pm 0.01$ \\
\hline & 3 & $2.048 \mathrm{E}-01 \pm 0.52 \%$ & $2.195 \mathrm{E}-01 \pm 0.49 \%$ & $1.07 \pm 0.01$ & $2.202 \mathrm{E}-01 \pm 0.32 \%$ & $1.00 \pm 0.01$ \\
\hline \multirow{3}{*}{$\mathrm{C}$} & 1 & $1.474 \mathrm{E}-01 \pm 0.48 \%$ & $1.598 \mathrm{E}-01 \pm 0.58 \%$ & $1.08 \pm 0.01$ & $1.588 \mathrm{E}-01 \pm 0.37 \%$ & $1.01 \pm 0.01$ \\
\hline & 2 & $2.674 \mathrm{E}-01 \pm 0.44 \%$ & $3.044 \mathrm{E}-01 \pm 0.42 \%$ & $1.14 \pm 0.01$ & $3.067 \mathrm{E}-01 \pm 0.30 \%$ & $0.99 \pm 0.01$ \\
\hline & 3 & $3.947 \mathrm{E}-01 \pm 0.46 \%$ & $4.510 \mathrm{E}-01 \pm 0.35 \%$ & $1.14 \pm 0.01$ & $4.513 \mathrm{E}-01 \pm 0.23 \%$ & $1.00 \pm 0.004$ \\
\hline \multirow{3}{*}{$\mathrm{D}$} & 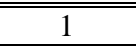 & 6.503E-02 $\pm 1.28 \%$ & $6.920 \mathrm{E}-02 \pm 0.87 \%$ & $1.06 \pm 0.02$ & 6.913E-02 $\pm 0.56 \%$ & $1.00 \pm 0.01$ \\
\hline & 2 & 7.393E-02 $\pm 1.30 \%$ & $7.644 \mathrm{E}-02 \pm 0.82 \%$ & $1.03 \pm 0.02$ & $7.820 \mathrm{E}-02 \pm 0.55 \%$ & $0.98 \pm 0.01$ \\
\hline & 3 & $3.329 \mathrm{E}-01 \pm 0.52 \%$ & $3.752 \mathrm{E}-01 \pm 0.38 \%$ & $1.13 \pm 0.01$ & $3.767 \mathrm{E}-01 \pm 0.24 \%$ & $1.00 \pm 0.005$ \\
\hline
\end{tabular}

Also included in Table 11 is a comparison between the forward multi-group CAAS detector response and the adjoint multi-group CAAS detector response. The agreement between these multi-group CAAS detector responses is almost perfect, which indicates that the adjoint calculations have been performed correctly.

When comparing the multi-group results to the continuous-energy results, only one change has occurred as far as the alarming of the detectors is concerned. Based on the multi-group results, detector 1 will alarm for source C. This was not the case for the continuous-energy simulation of this source detector pair.

\subsubsection{Approach 6: Adjoint Simulation, Analog MC, Mesh Tally}

One adjoint simulation would be performed for each detector location. The adjoint source would be the detector response function emitted from the detector location. A mesh tally would be made over the entire facility using the accident spectrum as the adjoint tally response function. The result of the mesh tally would have units of rem and any value greater than 0.150 rem would indicate that the detector would alarm for a source at that location.

\subsubsection{Approach 7: Adjoint Simulation, CADIS, Standard Tallies}

This analysis would be similar to approach 5, but with weight windows and a consistent biased source developed from a forward deterministic transport calculation. There would need to be one Monte Carlo calculation for every detector/accident combination.

\subsubsection{Approach 8: Adjoint Simulation, FW-CADIS, Mesh Tally}

This analysis would be similar to approach 6, but with weight windows and a consistent biased source developed with an FW-CADIS-like approach. Adjoint fluxes calculated by a deterministic transport calculation would be used to weight the sources of a forward deterministic transport calculation. The forward fluxes would be used to make the weight windows and consistent biased source for the final adjoint Monte Carlo calculation.

\subsubsection{Adjoint Approaches with Denovo-Only Calculations}

For CAAS detector placement studies, adjoint Monte Carlo calculations would be quite useful in determining the area of coverage for a particular detector location. In SCALE 6.1, the fixed-source 
Monte Carlo transport code Monaco does not contain a full adjoint capability. This capability has been developed for neutron transport, but not photon transport. As a proof of principal, the detector placement approaches previously discussed are evaluated using coarse (approximate) Denovo adjoint calculations in this section. The full input files associated with this analysis are provided in Appendix A.23.

The Denovo deterministic radiation transport code can be used (through the MAVRIC sequence) to perform both forward and adjoint calculations as a proof of principal for the adjoint detector placement approaches discussed in the proceeding sections. To obtain accurate solutions for large problems with a discrete ordinates code such as Denovo, fine meshes, large quadrature orders, large amounts of memory and long computation times would be required. This is probably not a reasonable solution method for realistic CAAS placement studies. However, deterministic-based solutions could still be used for scoping studies where detailed accuracy is not needed. For CAAS studies using Denovo, SCALE 6.1 requires several utilities to work with the Denovo output files.

To demonstrate the use of Denovo, consider the simple problem introduced at the beginning of Section 9. Recall, the mesh is fairly coarse $\left(112 \times 89 \times 33 \approx 0.3 \times 10^{6}\right.$ voxels $)$ and consists of planes with spacing less than $30.48 \mathrm{~cm}$ that delineates all of the important concrete and steel surfaces. This is the same mesh used by Denovo in the importance maps for the CADIS and FW-CADIS SCALE and MCNP CAAS calculations in Section 9.1. Figure 37 shows the Denovo mesh over one corner of the storage room model. Concrete walls (grey), steel rack structures (blue) and homogenized $\mathrm{UO}_{2}$ /air (light yellow) in the double-sided storage bins are shown.

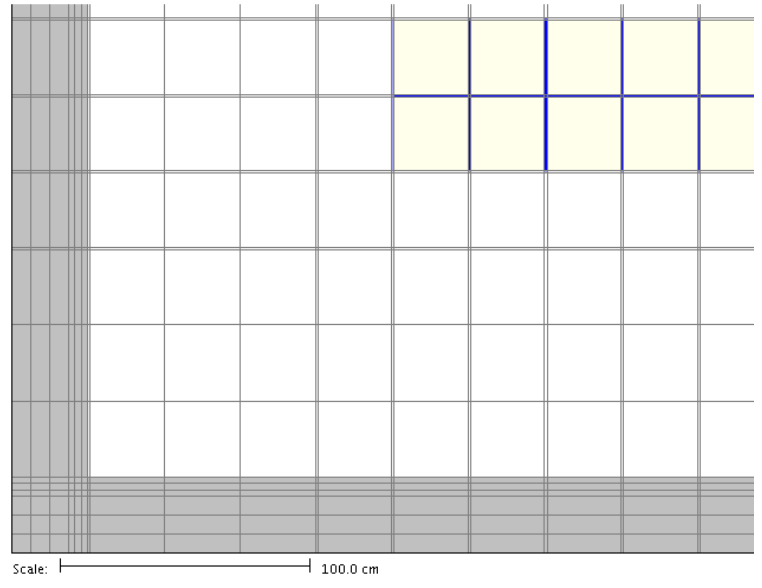

Figure 37. Portion of the Denovo mesh showing material.

MAVRIC has been used to perform an adjoint Denovo calculation for each detector location, using the dose response as the source spectrum, an S8 quadrature, P3 Legendre representation of scattering, and the ENDF/B-VII.0 27-neutron/19-photon group cross-section library. The only result of a Denovo calculation available through SCALE is the scalar flux. The scalar fluxes produced when the adjoint source is located in detector 1 are shown in Figure 38. The adjoint scalar fluxes in Figure 38 have been convolved with the accident source spectrum and multiplied by the appropriate normalization constants to create a map of response for detector 1 for a source at any location. This map of CAAS detector 1 response is shown in Figure 39. Note that the mesh viewer is hard-wired to add the title "Adjoint Neutron Flux,” even though Figure 39 maps CAAS detector response for a source at any given position. 


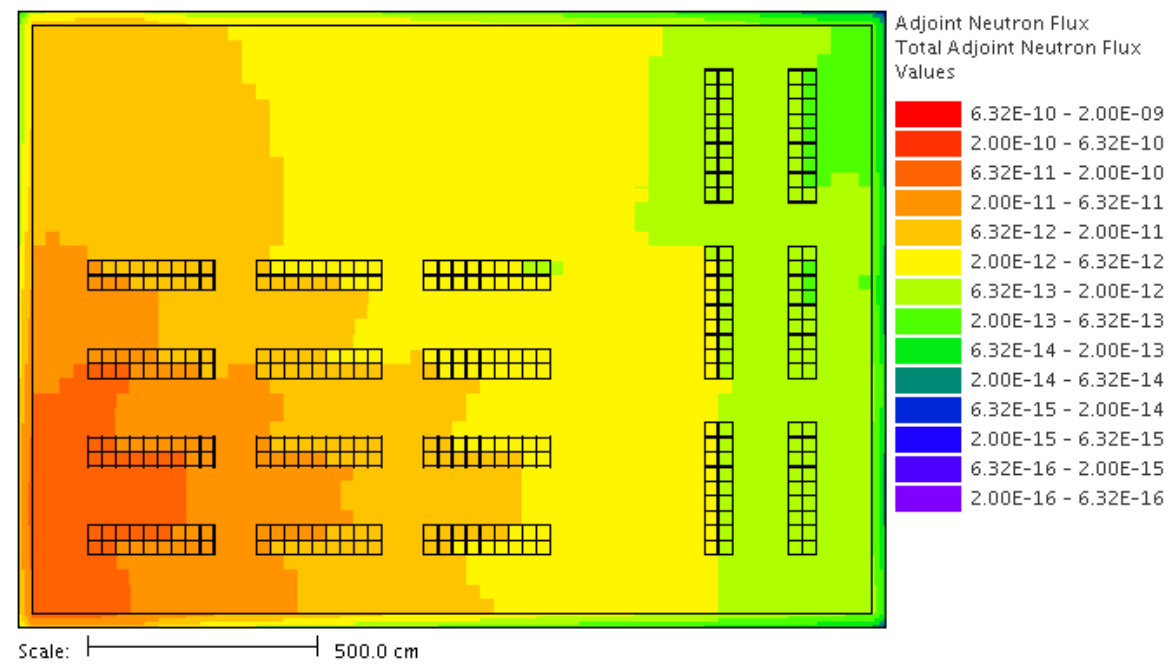

Figure 38. Total adjoint scalar flux as computed by Denovo with source in detector $1(\mathrm{z}=100 \mathrm{~cm})$.

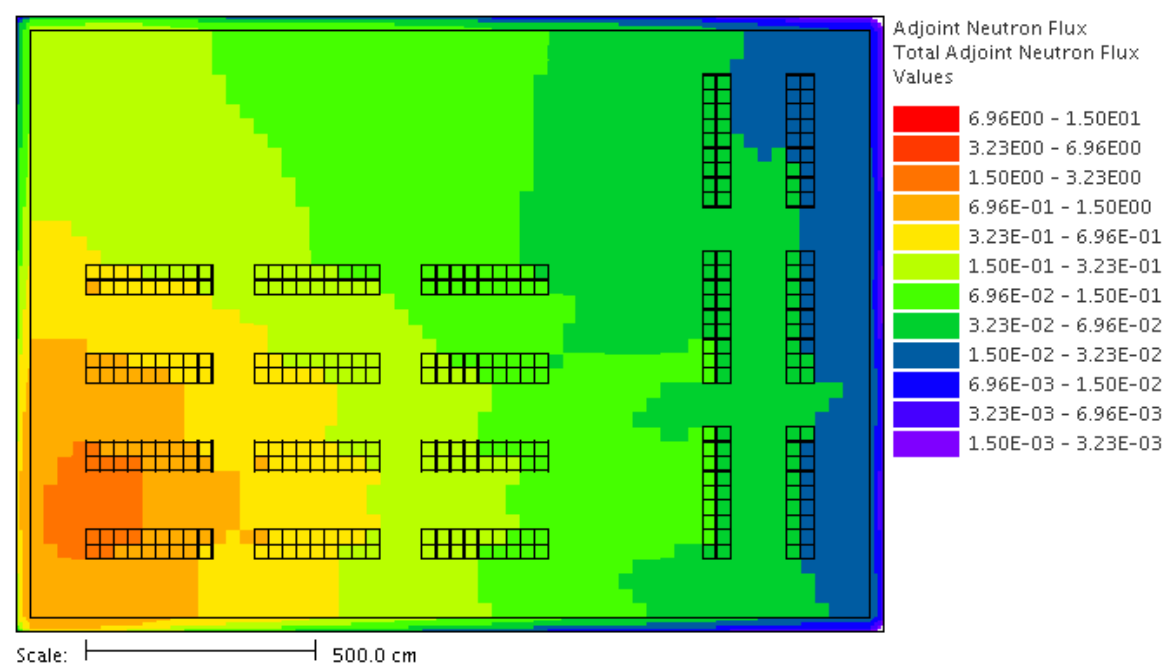

Figure 39. CAAS detector 1 response map for a source anywhere $(\mathrm{z}=100 \mathrm{~cm})$.

The values of the CAAS detector responses at the detector locations in Figure 18 were evaluated for all four accident sites, and these are shown in Table 12. The CAAS detector responses calculated by Denovo for source and detector locations in close proximity are similar to the SCALE Monte Carlo results. For combinations of source detector locations farther apart, the Denovo results do not agree as well with the Monte Carlo results, which likely is due to the approximate nature of the Denovo solution (course mesh, quadrature, and Legendre representation of the cross-section data).

Table 12. CAAS detector response (rem) calculated using adjoint Denovo (highlighted doses are above the 0.150 rem CAAS alarm set point).

\begin{tabular}{|c||c|c|c|}
\hline Source & Detector 1 & Detector 2 & Detector 3 \\
\hline \hline A & $1.694 \mathrm{E}-01$ & $8.823 \mathrm{E}-02$ & $7.251 \mathrm{E}-02$ \\
\hline B & $1.770 \mathrm{E}-01$ & $1.608 \mathrm{E}-01$ & $1.352 \mathrm{E}-01$ \\
\hline C & $8.815 \mathrm{E}-02$ & $1.775 \mathrm{E}-01$ & $3.068 \mathrm{E}-01$ \\
\hline D & $2.769 \mathrm{E}-02$ & $3.235 \mathrm{E}-02$ & $2.611 \mathrm{E}-01$ \\
\hline
\end{tabular}


Utilities similar to those mentioned in 9.1.5 that filter and add mesh tallies have been used to filter and add the Denovo scalar flux. Figure 39 has been filtered to show areas where the CAAS Detector response is above the 0.150 rem alarm set point, which is displayed in Figure 40. The response for detectors 2 and 3, like that for detector 1 in Figure 39, has been filtered to create Figure 41 and Figure 42, respectively. The three filtered CAAS detector responses in the figures below show the "zones of coverage" for each of the three detectors in Figure 18 at $\mathrm{z}=100 \mathrm{~cm}$.

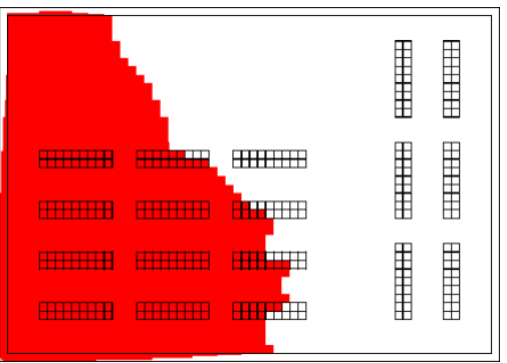

Figure 40. Detector 1 coverage.

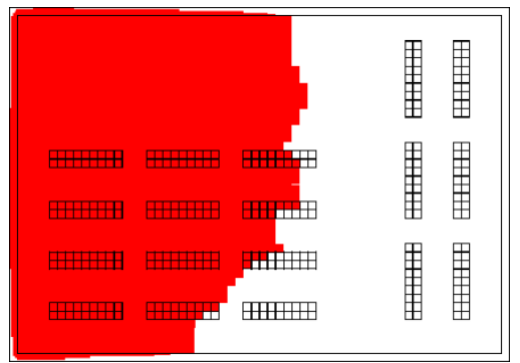

Figure 41. Detector 2 coverage.

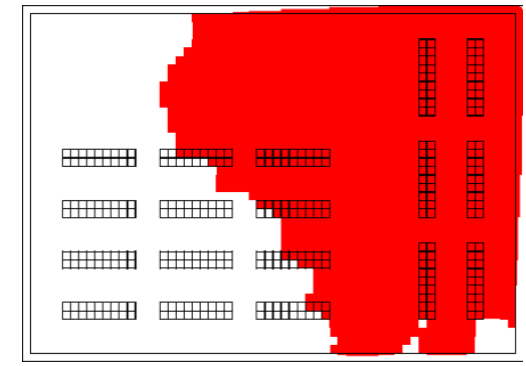

Figure 42. Detector 3 coverage.

The coverage areas for each detector have been added together to find the number of detectors that alarmed for an accident in any location. This result is shown in Figure 43 (again, the title that the viewer shows is incorrect). For facilities that require coverage of all credible accidents by at least two CAAS units, Figure 43 shows that only the left half of the storage room (yellow and red areas) qualifies. Accidents in the green areas will only alarm one detector. Accidents in the purple areas will not alarm any of the three detectors.

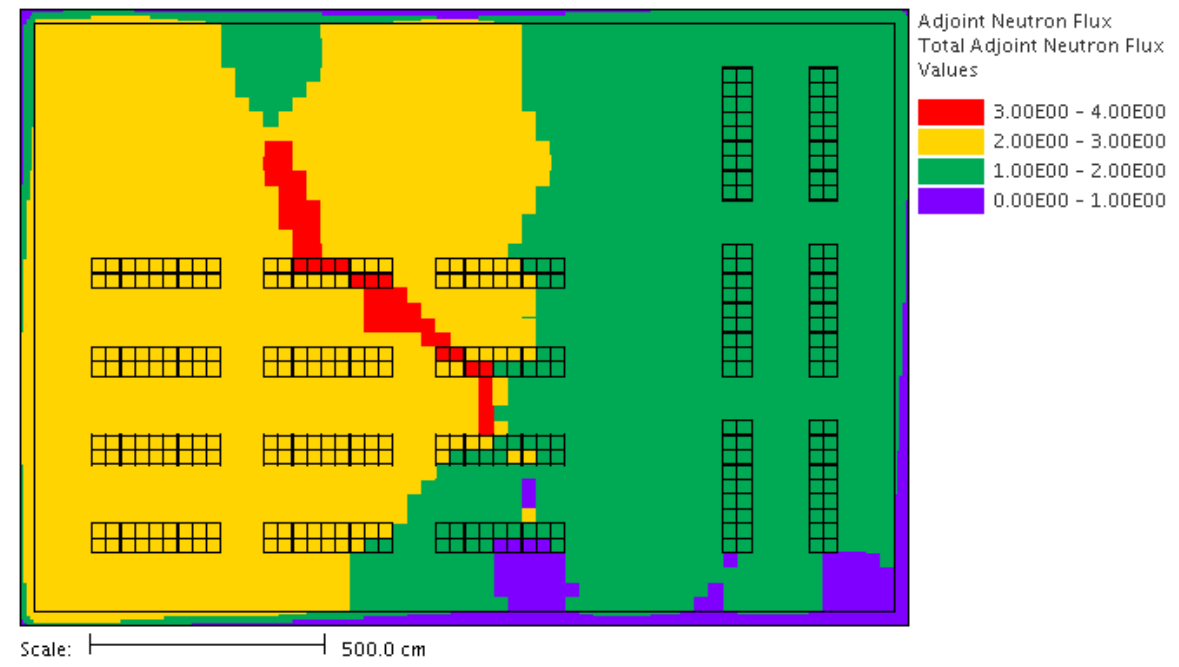

Figure 43. How many detectors are triggered for an accident at any location $(\mathrm{z}=100 \mathrm{~cm})$.

One can see that adjoint transport simulations are very useful to understanding the coverage of a CAAS detector in a specific location for any credible accident. Monte Carlo adjoint simulations reduce the amount of memory required to perform this sort of analysis, as compared to a deterministic calculation. Adjoint Monte Carlo calculations could be accelerated using an automated variance reduction technique analogous to FW-CADIS for forward calculations with mesh tallies. 


\section{SUMMARY AND CONCLUSIONS}

This document has provided an overview of ANSI/ANS-8.3-1997 (R2012) as it relates to computational analysis of CAAS detector responses. In particular, the process of determining the minimum accident of concern has been discussed. Subsequently, the document provides basic examples of how to calculate CAAS detector responses using the 3D Monte Carlo radiation transport capabilities of SCALE and MCNP. Then, an example of how to calculate the coverage of a CAAS detector has been provided using SCALE and MCNP. Finally, a strategy to determine effective CAAS detector location has been provided. This strategy accounts for the relative number of credible accident locations and CAAS detectors, and recommends applying forward and adjoint transport simulations in different situations. The forward transport methods currently available are much more mature than the adjoint transport methods, but adjoint transport results can provide increased computational efficiencies in situations where there are more accident locations than detector locations or when the accident locations are unknown.

\section{ACKNOWLEDGEMENTS}

The authors have a number of people to acknowledge. Robert Wilson of DOE/EM-41 provided the motivation and support to produce this guidance document, and Jerry McKamy of the DOE NCSP provided methods development support for some of the computational tools applied in this document. Larry Wetzel at Babcock \& Wilcox, Lynchburg, Virginia, provided support and assistance developing the example discussed in Section 9, and also served as an external reviewer of the document. The program manager at ORNL for this work was Mike Dunn, who was also an internal reviewer. Other external reviewers include Shivi Singh, Larry Berg, and Andy Prichard. Additional internal reviewers at ORNL include Doug Bowen, Justin Clarity, Don Muller, Billy Lee, Mike Westfall, and Joel Risner. Hannah Turpin and Angie Alford of ORNL provided editorial assistance and formatted the final document. The authors would like to thank all of these people for their support and recommendations, which were very helpful in preparing this document.

\section{REFERENCES}

1. “ANSI/ANS-8.3-1997 (R2012) Criticality Accident Alarm System,” American Nuclear Society, La Grange Park, IL (2012).

2. "SCALE: A Modular Code System for Performing Standardized Computer Analysis for Licensing and Evaluations,” ORNL/TM-2005/39, Version 6.1, Vols. I - III, Oak Ridge National Laboratory (June 2011).

3. X-5 Monte Carlo Team, "MCNP-A General Monte Carlo N-Particle Transport Code, Version 5," Los Alamos National Laboratory, LA-UR-03-1987 (Revised 2008).

4. ICRU-44, “Tissue Substitutes in Radiation Dosimetry and Measurement,” International Commission on Radiation Units and Measurements, Bethesda, MD (1989).

5. ICRU-57, "Conversion Coefficients for Use in Radiological Protection Against External Radiation," International Commission on Radiation Units \& Measurements, Bethesda, MD (1998).

6. N. M. Greene, L. M. Petrie, and M. L. Williams, "XSDRNPM: A One-Dimensional DiscreteOrdinates Code for Transport Analysis,” ORNL/TM-2005/39, Version 6.1, Section F3, Oak Ridge National Laboratory (June 2011). 
7. R. E. Alcouffe, R. S. Baker, J. A. Dahl, S.A. Turner, and R. Ward, "PARTISN: A Time-Dependent, Parallel Neutral Particle Transport Code System,” LA-UR-08-07258, Los Alamos National Laboratory (Revised Nov. 2008).

8. T. M. Evans, A. S. Stafford, R. N. Slaybaugh, and K. T. Clarno, "Denovo: A New Three-Dimensional Parallel Discrete Ordinates Code in SCALE,” Nucl. Technol., 171, pp. 171-200 (2010).

9. S. Goluoglu, L. M. Petrie, M. E. Dunn, D. F. Hollenbach, and B. T. Rearden, "Monte Carlo Criticality Methods and Analysis Capabilities in SCALE,” Nucl. Technol., 174, pp. 214-235 (2011).

10. D. E. Peplow, “Monte Carlo Shielding Analysis Capabilities with MAVRIC,” Nucl. Technol., 174, pp. 289-313 (2011).

11. International Handbook of Evaluated Criticality Safety Benchmark Experiments, NEA/NSC/DOC(95)03, Organization for Economic Co-operation and Development-Nuclear Energy Agency (OECD-NEA) (September 2012).

12. T. M. Miller and D. E. Peplow, "Corrected User Guidance to Perform Three-Dimensional Criticality Accident Alarm System Modeling with SCALE,” Transactions of the American Nuclear Society, 108, p. 498 (2013).

13. J. C. Wagner, D. E. Peplow, and S. W. Mosher, "FW-CADIS Method for Global and Semi-Global Variance Reduction of Monte Carlo Radiation Transport Calculations," accepted by Nuclear Science and Engineering, 177 (2013).

14. J. C. Wagner, “An Automated Deterministic Variance Reduction Generator for Monte Carlo Shielding Applications," Proceedings of the American Nuclear Society 12th Biennial RPSD Topical Meeting, Santa Fe, New Mexico, April 14-18 (2002).

15. C. M. Hopper, B. L. Broadhead, R. L. Childs, and J. S. Tang, "An Updated Nuclear Criticality Slide Rule,” ORNL/TM-13322 Vols. 1 and 2, NUREG/CR-6504 Vols. 1 and 2, Oak Ridge National Laboratory (Vol. 1 April 1997, Vol. 2 April 1998).

16. ANS-6.1.1 Working Group, M. E. Battat (Chairman), “American National Standard Neutron and Gamma-Ray Flux-to-Dose Rate Factors,” ANSI/ANS-6.1.1-1977 (N666), American Nuclear Society, LaGrange Park, Illinois (1977).

17. J. C. Wagner, et. al., “MCNP: Multigroup / Adjoint Capabilities,” LA-12704, Los Alamos National Laboratory (1994). 


\section{APPENDIX A}

\section{SCALE 6.1 AND MCNP5 INPUT FILES}

The SCALE and MCNP input files in this appendix contain all of the input necessary to run the calculations described in the main body of this report. If the SCALE CAAS analysis capability has been applied, that specific input file contains all of the required KENO, MT2MSM, and MAVRIC/Monaco input. The KENO and MT2MSM part could have been run only once, and the results produced by those codes read in by any MAVRIC/Monaco calculation. For completeness, the KENO and MT2MSM input have been included with each MAVRIC/Monaco input that applied the CAAS analysis option. For MCNP calculations that used ADVANTG, the input file for the beta version of ADVANTG 3.0 is also included. However, the weight window files (wwinp) and biased source created by ADVANTG are supplied with the MCNP inputs. Below is a listing of all the input files that are provided along with a brief description. The actual input files are available for download in the publications section of the SCALE website (http://scale.ornl.gov/) or directly from the report authors.

\begin{tabular}{|c|c|}
\hline $\begin{array}{c}\text { Appendix } \\
\text { Number }\end{array}$ & Description \\
\hline A.1 & Jezebel Eigenvalue Benchmark: SCALE Input \\
\hline A.2 & Jezebel Plus Spherical CAAS Detector: SCALE Input \\
\hline A.3 & Jezebel Eigenvalue Benchmark: MCNP Input \\
\hline A.4 & Jezebel Plus Spherical CAAS Detector: MCNP Input \\
\hline A.5 & Jezebel in Block Building Minimum Accident: SCALE Input \\
\hline A.6 & Jezebel in Block Building Minimum Accident: MCNP Input \\
\hline A.7.a & Jezebel in Block Building Spherical CAAS Detector Response: SCALE Input \\
\hline A.7.b & Jezebel in Block Building Spherical CAAS Detector Response: SCALE Input with FW-CADIS \\
\hline A.8 & Jezebel in Block Building Spherical CAAS Detector Response: MCNP Input \\
\hline A.9.a & Jezebel Leakage Spectra: SCALE Input \\
\hline A.9.b & Jezebel as a Point Source in Block Building Spherical CAAS Detector Response: SCALE Input \\
\hline A.10.a & Jezebel Leakage Spectra: MCNP Input \\
\hline A.10.b.1 & $\begin{array}{l}\text { Jezebel as a Point Source in Block Building Spherical CAAS Detector Response: MCNP Input with Neutron } \\
\text { Source }\end{array}$ \\
\hline A.10.b.2 & Jezebel as a Point Source in Block Building Spherical CAAS Detector Response: MCNP Input with Photon Source \\
\hline A.13 & Jezebel as a Point Source in Block Building Mesh Tally of Detector Response: SCALE Input \\
\hline A.14.a & Jezebel as a Point Source in Block Building Mesh Tally of Detector Response: MCNP Input with Neutron Source \\
\hline A.14.b & Jezebel as a Point Source in Block Building Mesh Tally of Detector Response: MCNP Input with Photon Source \\
\hline A.15 & Jezebel as a Point Source in Block Building Adjoint Denovo Calculation of Detector Response: SCALE Input \\
\hline A.16 & vsResp Input to Convert Denovo Scalar Fluxes to CAAS Detector Response \\
\hline A.17 & Placement Analysis Approach 1: SCALE Input \\
\hline A.18 & Placement Analysis Approach 2: SCALE Input \\
\hline A.19.a & Placement Analysis Approach 3: SCALE Input \\
\hline A.19.b & Placement Analysis Approach 3: MCNP Input \\
\hline A.20.a & Placement Analysis Approach 4: SCALE Input \\
\hline A.20.b & Placement Analysis Approach 4: MCNP Input \\
\hline A.21 & Placement Analysis Approach 5: MCNP Forward Multi-group Input \\
\hline A.22 & Placement Analysis Approach 5: MCNP Adjoint Multi-group Input \\
\hline A.23 & Adjoint Placement Analysis Approach with Denovo \\
\hline
\end{tabular}




\section{APPENDIX B}

In order to simplify the discussion of modeling a criticality accident (e.g., Jezebel or PU-MET-FAST001) as a point with SCALE in Section 7.3 of the main report, some of the details have been moved to Appendix B. Appendix B.1 evaluates the validity of reducing Jezebel to a point source in Section 7.3. Appendix B.2 presents how to calculate Jezebel's neutron and photon leak spectra using Monaco, which were used in Section 7.3 to calculate the response of a CAAS detector.

\section{APPENDIX B.1}

\section{DETAILED EVALUATION OF SIMPLIFYING THE JEZEBEL CRITICAL ASSEMBLY TO A POINT SOURCE}

First consider the conditions listed in Section 6 to determine if the problem is suitable for this simplification. The detector geometry is a significant distance from the source geometry, greater than 10 times the diameter. This is illustrated by Table B.1, which shows the distance between a few points within the source and detector geometries. Some of the location names in Table B.1 (like Top or Right) should be thought of in the context of Figure 5 in the main text. In Figure 5, Jezebel (red sphere) is in the left room and the CAAS detector (purple sphere) is in the right room. Therefore, the right of the source geometry is the location on Jezebel nearest the CAAS detector, and the left of the detector geometry is the location on the CAAS detector nearest Jezebel. The top of either geometry is nearest the ceiling and the bottom of either geometry is nearest the floor.

Table B.1. Distances between key locations within the source and detector geometries.

\begin{tabular}{|l|l|l|}
\hline Location within Source Geometry & Location with Detector Geometry & Distance Between Locations (cm) \\
\hline Center: $(280,300,100)$ & Center: $(1129,71,269)$ & 895.4 \\
\hline Bottom: $(280,300,93.61507)$ & Top: $(1129,71,299.48)$ & 903.1 \\
\hline Right: $(286.38493,300,100)$ & Left: $(1098.52,71,269)$ & 860.6 \\
\hline Left: $(273.51607,300,100)$ & Right: $(1159.48,71,269)$ & 930.6 \\
\hline
\end{tabular}

Based on the fact that all of the sampled distances between the source and detector are much larger than 10 times the diameter of Jezebel, the first condition listed in Section 6 is satisfied.

The second condition in Section 6 is satisfied as well. The volume of Jezebel $\left(\sim 1090 \mathrm{~cm}^{3}\right)$ is less than $0.002 \%$ of the total volume of the left room $\left(7.488 \times 10^{7} \mathrm{~cm}^{3}\right)$ in Figure 5 . Therefore, the probability a particle will leak from Jezebel, scatter within the block building in Figure 5, and then interact with Jezebel again is very small. The nearest Jezebel is to any reflecting object in the block building is approximately $87 \mathrm{~cm}$, which is the distance from the bottom of Jezebel to the concrete floor. This distance can be judged using the same criteria as the first condition; the distance should be greater than 7 to 10 times the most pertinent dimension of the critical assembly. In this case, $87 \mathrm{~cm}$ is about 7 times the diameter of the Jezebel, which is adequate for this geometry. This shows that the recommendation of 7 to 10 times the pertinent dimension of the critical assembly is just a suggested rule-of-thumb, not a hard requirement.

\section{APPENDIX B.2}

\section{DETAILS OF HOW TO CALCULATE LEAKAGE FROM A CRITICAL ASSEMBLY USING MONACO}

One can start with the SCALE input files used in Section 7.1. The KENO and MT2MSM input will not change. The geometry in the MAVRIC input file needs to change significantly. The MAVRIC geometry should include only Jezebel and a spherical shell surrounding Jezebel, and all materials other than the 
plutonium mixture can be deleted. The neutron and photon spectra will be tallied within the spherical shell surrounding Jezebel. The full input file for this analysis is included in Appendix A.9.a. The lines below show the new MAVRIC geometry in its entirety.

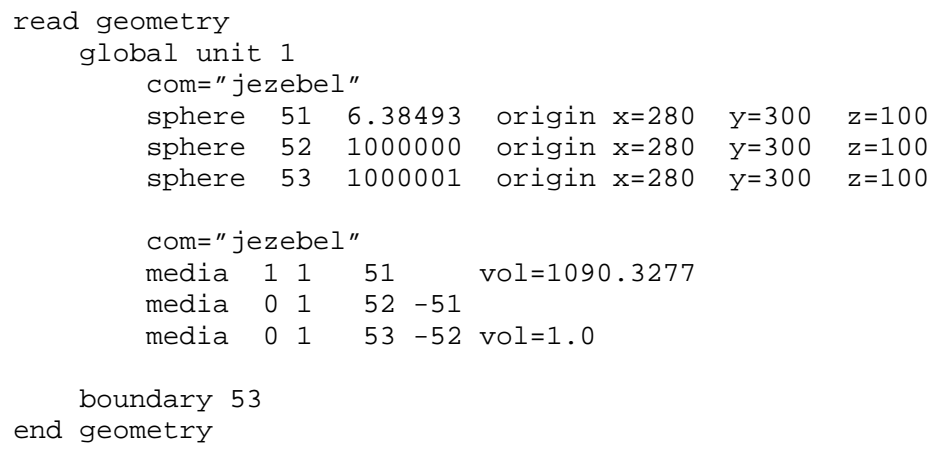

There are a few particular features of this new geometry that warrant more discussion. Before that discussion begins, first note that the SCALE Monte Carlo code Monaco does not have an actual particle current tally. Monaco only has tallies that calculate the flux within a volume, at a point, or within a Cartesian or cylindrical mesh. To calculate the flux within a volume, a Monte Carlo code can use the following track length estimator:

$$
\text { Flux }=\frac{1}{N} \sum_{N} \frac{W * T_{L}}{V},
$$

where $\mathrm{W}$ is the weight of the particle, $\mathrm{T}_{\mathrm{L}}$ is the track length of the particle within the region in which the flux is being tallied, $\mathrm{V}$ is the volume of that region, and $\mathrm{N}$ is the number of histories. However, what is really needed is not the flux of particles exiting Jezebel, but rather the current or number of particles exiting Jezebel as a function of energy. To calculate the current with a Monte Carlo code the following estimator is used:

$$
\text { Current }=\frac{1}{N} \sum_{N} W .
$$

The difference in these two expressions for flux and current produce two peculiarities in the new MAVRIC geometry, which are discussed next. If the track length and volume in the flux estimate are both equal to 1.0, the flux and current will be equal. The volume of the spherical shell in which the neutron and photon leakage spectra are tallied is easily set to 1.0 on the media cards defining the geometry, regardless of what the actual volume is. Forcing the track length to equal 1.0 requires that the spherical shell be a very large distance away from the outer surface of Jezebel and that the thickness of the spherical shell be $1.0 \mathrm{~cm}$. This is accomplished by making the inner radius of the spherical shell $1 \times 10^{6} \mathrm{~cm}$ and the outer radius of the spherical shell $1.000001 \times 10^{6} \mathrm{~cm}$. It is important to emphasize that the regions outside of Jezebel must be empty (i.e. void). Otherwise, these materials will scatter particles that have leaked from Jezebel and change the spectra before reaching the spherical shell. The source strength in this calculation should be set to 1 fission per second, and the response IDs and multiplier should be deleted from the region tally. Also, the region number in the tally should be set to match that of the spherical shell.

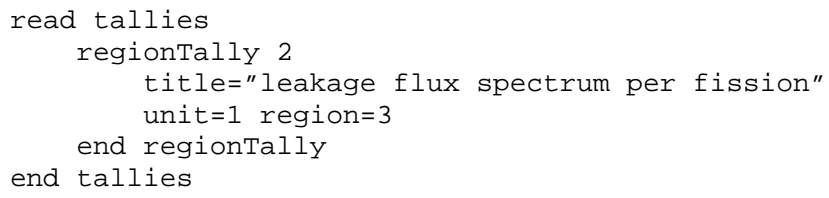


As before, the "noFissions" keyword must be used because this system is critical, and the "noSecondaries" keyword is not used because photons are being tallied as well as neutrons. The numbers of neutrons and photons that leaked from Jezebel as calculated by SCALE are given below in Table B-1. The spectra are plotted along with the MCNP results in Figure 7 and Figure 8.

Table B-1. Total leakage from Jezebel as calculated by SCALE (relative errors are at the 1- $\sigma$ level).

\begin{tabular}{|l|l|}
\hline Neutrons per fission per second & $2.12641 \mathrm{E}+00 \pm 0.007 \%$ \\
\hline Photons per fission per second & $5.87772 \mathrm{E}-01 \pm 0.04 \%$ \\
\hline
\end{tabular}




\section{APPENDIX C}

\section{ADDITIONAL DETAILS CONCERNING MCNP MULTI-GROUP ADJOINT TRANSPORT CALCULATIONS}

The use of fixed-source adjoint transport analysis relies on the following relationship:

$$
R=\int_{V_{d}} \int_{E} \sigma_{d}(\vec{r}, E) \phi(\vec{r}, E) d E d V=\int_{V_{S}} \int_{E} q(\vec{r}, E) \phi^{\dagger}(\vec{r}, E) d E d V,
$$

where $\mathrm{R}$ is the calculated detector response, $V_{d}$ is the detector volume, $\sigma_{d}$ is the detector response function (flux-to-dose-rate conversion factors), $\phi$ is the forward scalar flux, $V_{s}$ is the source volume, $q$ is the forward source, and $\phi^{\dagger}$ is the adjoint scalar flux.

In a forward Monte Carlo calculation, forward source particles are started in the forward source volume and are tracked to the detector volume. The forward source particles that make it to the detector volume contribute to the tally of the forward scalar flux. The forward scalar flux is convolved with the flux-todose-rate conversion factors to calculate the CAAS detector response. Forward Monte Carlo calculations are the types of calculations that criticality safety practitioners have a lot of experience with from calculating $\mathrm{k}_{\mathrm{eff}}$.

Alternatively, in an adjoint Monte Carlo calculation, adjoint source particles are started in the detector volume and are tracked to the forward source volume. The adjoint source particles that make it to the forward source volume contribute to the tally of the adjoint scalar flux. The adjoint scalar flux is convolved with the forward source to calculate the CAAS detector response.

The steps that must be taken to convert a forward MCNP input file to an equivalent adjoint MCNP input file are outlined below. This outline assumes that the "WGT" variable on the SDEF card is equal to 1.0, the system source strength is set via the FM tally multiplier card, and that the default MCNP multi-group cross sections are being used (MGXSNP, 30 neutron groups and 12 photon groups, based on ENDF/B-V).

1) Create adjoint sources that correspond to each forward tally

a) The spatial domain of the adjoint source should match the spatial domain of the forward tally

b) The forward detector response function (flux-to-dose-rate conversion factors) should be used as the energy distribution of adjoint source particles

2) Create adjoint tallies that correspond to each forward source

a) The spatial domain of the adjoint tally should match the spatial domain of the forward source

b) The forward source particle energy distribution should be used as the adjoint detector response function (flux-to-dose-rate conversion factors)

3) Properly set the tally multiplier for each adjoint tally

a) Initially, set the adjoint tally multiplier equal to the forward tally multiplier (however, some additional normalization is needed)

b) The forward detector response function will be normalized to an area of 1.0 as the adjoint source energy distribution (a pdf), so multiply the adjoint tally multiplier by the integral of the adjoint source energy distribution (for a histogram, this is the sum of all of the bins)

c) The forward source particle energy distribution will not be normalized to an area of 1.0 as the adjoint detector response function, so divide the adjoint tally multiplier by the integral of the adjoint detector response function

4) Select the multi-group cross sections and set the forward or adjoint transport mode using the MGOPT card in the data card section of the input (see material specifications)

a) mgopt A 12 - adjoint photon transport 
b) mgopt A 30 - adjoint neutron transport

c) mgopt A 42 - adjoint coupled neutron/photon transport

5) Correctly set the particle energy cutoffs, which correspond to the maximum energy for an adjoint MCNP calculation (maximum energies for the MGXSNP cross sections are shown below)

a) cut:n j 17

b) cut:p j 20

6) MCNP5 release 1.60 multi-group adjoint calculations have produced bad trouble errors (absolute value of cosine in subroutine rotas is greater than 1.0) when a material that does not contain photon production data in the MGXSNP cross-section library is used. A specific example of this behavior was observed when using ${ }^{234} \mathrm{U}$. If this bad trouble error is encountered, consider removing the materials with no photon production data from the material models.

This information about converting a forward MCNP input to an equivalent adjoint MCNP will apply to most CAAS analysis models.

It needs to be pointed out that the group structure of the MGXSNP cross sections is rather coarse, and that these cross sections are infinitely dilute and have not been self-shielded. This means that these cross sections are most applicable to fast systems, and are likely to perform poorly for systems where resonance absorption is important. A best practice would be to generate problem dependent cross sections for each analysis performed and convert those to MCNP multi-group cross sections. The problem dependent multi-group cross sections could be generated with SCALE, but a code named CRSRD is required to write them to MCNP multi-group format. There are more details about this in Reference 17.

Before the multi-group MCNP calculations could be run, the fine-group source-particle energy distribution and flux-to-dose-rate conversion factors in the continuous-energy input files (Appendix A.19.b) were collapsed to the coarse group structure of the MGXSNP cross sections. These data could be collapsed a number of different ways, but only one approach for each data set is illustrated here.

Integrating over the fine group structure within each coarse group collapses the forward source particle energy distribution used in the continuous-energy calculation. Since this distribution is a histogram, this simply means the number of source particles in each fine group that falls within a coarse group will be summed to determine the number of source particles in the coarse group. However, to account for the different energies of particles between the fine and course group structure, a weighting will be applied to the summation, which corresponds to the ratio of the mean fine group energy to the mean coarse group energy. This weighting serves as an attempt to converse energy between the fine group structure and the coarse group structure. A mathematical expression of this algorithm is given below along with a diagram to visualize the change in group structure.

$$
\begin{aligned}
& \text { Fine Group Coarse Group } \\
& \text { Structure Structure } \\
& S_{G}=\sum_{i=g}^{g \prime} \frac{e_{i}+e_{i+1}}{E_{G}+E_{G+1}} s_{i}
\end{aligned}
$$

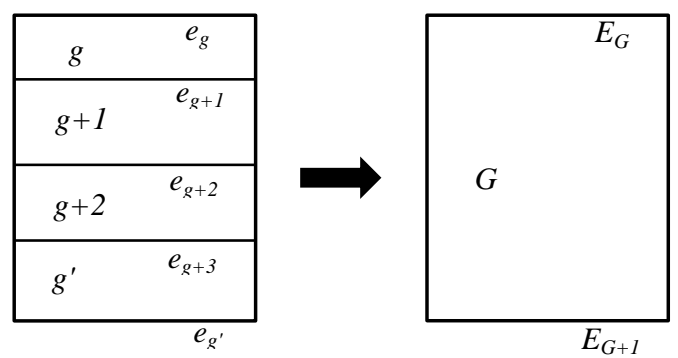

In the above equation, $S_{G}$ is the number of source particles in coarse group $G, s_{i}$ is the number of source particles in the $\mathrm{i}^{\text {th }}$ fine group, $e_{i}$ is the maximum energy of the $\mathrm{i}^{\text {th }}$ fine group, $e_{i+1}$ is the minimum energy of 
the $\mathrm{i}^{\text {th }}$ fine group, $E_{G}$ is the maximum energy of coarse group $G$, and $E_{G+1}$ is the minimum energy of coarse group $G$.

Finding the average flux-to-dose-rate conversion factor over the fine group structure within each coarse group collapses the forward flux-to-dose-rate conversion factors used in the continuous-energy calculation. Instead of calculating a standard arithmetic mean, a weighted mean will be calculated to determine the average flux-to-dose-rate conversion factor for each coarse group. The weighting is the fraction of the coarse group that each fine group covers, which corresponds to the ratio of the fine group width to the coarse group width. A mathematical expression of this algorithm is given below:

$$
\mathcal{R}_{G}=\sum_{i=g}^{g^{\prime}} \frac{e_{i}-e_{i+1}}{E_{G}-E_{G+1}} r_{i} .
$$

The flux-to-dose-rate conversion factor for coarse group $G$ is $\mathcal{R}_{G}$ and $r_{i}$ is the flux-to-dose-rate conversion factor for the $\mathrm{i}^{\text {th }}$ fine group.

In cases where $e_{g}$ and $E_{G}$ and/or $e_{g}$, and $E_{G+1}$ are not equal, the appropriate fraction of each fine group that overlaps the coarse group energy boundary is added to the correct coarse group. This is true for both of the algorithms that collapse the forward source particle energy distribution and the flux-to-dose-rate conversion factors.

Once these group collapse operations are complete, this data is used to complete step 3 of the conversion of a forward MCNP input to an adjoint MCNP input. All of step 3 can be represented with the following equation:

$$
F M_{\text {adjoint }}=F M_{\text {forward }} \sum_{i=G}^{G \prime} \frac{\mathcal{R}_{i}}{S_{i}} .
$$

FM refers to the MCNP tally multiplier and $\mathcal{R}$ and $S$ refer to the expressions above. Keep in mind that $S$ is the forward source particle energy distribution, which becomes the adjoint flux-to-dose-rate conversion factors, and $\mathcal{R}$ is the forward flux-to-dose-rate conversion factors, which become the adjoint source particle energy distribution. 